\title{
Characterization and comparative
}

\author{
analyses of type $\mathrm{Vd}$-secreted
}

\section{phospholipases expressed in pathogenic}

\section{bacteria}

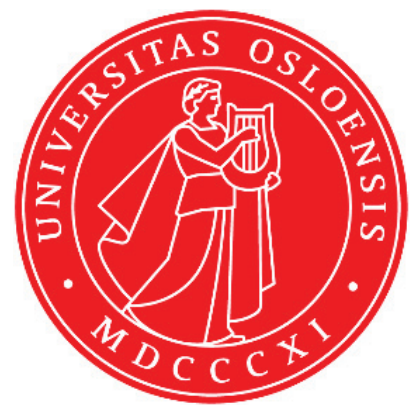

Thomas Trunk

Faculty of Mathematics and Natural Sciences

University of Oslo

A thesis submitted for the degree of

Doctor of Philosophy 
(C) Thomas Trunk, 2020

Series of dissertations submitted to the Faculty of Mathematics and Natural Sciences, University of Oslo No. 2262

ISSN $1501-7710$

All rights reserved. No part of this publication may be reproduced or transmitted, in any form or by any means, without permission.

Cover: Hanne Baadsgaard Utigard.

Print production: Reprosentralen, University of Oslo. 


\section{Acknowledgments}

The main part of my research was conducted at the department of bioscience at the University of Oslo and funded by the Norwegian Research Council (NRC). Additional institutions to support my research were the department of microbiology and hygiene at the Eberhard Karls University in Tübingen (Germany), as well as at the Virginia Polytechnic Institute and State University (US), funded by SFB766 and UiO respectively. I am grateful to UiO, the Eberhard Karls University of Tübingen, Virginia Tech, NRC and SFB for the trust and support.

I would like to express my sincere gratitude to my supervisors, Jack C. Leo, Prof. Dirk Linke and Prof. Melinka Butenko, for their encouragement, support and guidance throughout my PhD. Special thanks to Jack for his continuous patience, motivation, optimism and immense knowledge! Your encouragement allowed me to grow as scientist and I couldn't have imagined a better boss. Thanks for all you have done!

Thanks to the bunch of beautifully weird and kind people that started as colleagues and became friends over the last couple of years. Thanks to Marcella Orwick Rydmark, Athanasios Saragliadis, Kenneth Schneider, Daniel Hatlem, Agnieszka Wrobel, Krystyna Liskewicz, Hawzeen Salah Khalil, 
Nadeem Joudeh and Ina Meuskens. You were quick to welcome me to the group for which I will always be grateful.

Thanks to Daniel, Ina, Chris Hadjineophytou, Siri Gusland and Nadeem for being amazing friends. You made my time in Oslo unforgettable.

Special thanks also to Liz for her patience and support during my time of writing. You are the best that ever happened to me! 


\section{List of papers}

1. Klein K, Sonnabend MS, Frank L, Leibiger K, Franz-Wachtel M, Macek B, Trunk T et al. Deprivation of the Periplasmic Chaperone SurA Reduces Virulence and Restores Antibiotic Susceptibility of Multidrug-Resistant Pseudomonas aeruginosa. Front Microbiol. 2019;10:100.

2. Trunk T, Casasanta MA, Yoo CC, Slade DJ, Leo JC. Comparison of type $5 d$ autotransporter phospholipases demonstrates a correlation between high activity and intracellular pathogenic lifestyle. Biochem J. 2019;476(18):2657-76.

3. Trunk T, Morth JP, Leo JC. Low resolution structure of the phospholipase autotransporter of Aeromonas hydrophila. Manuscript 



\section{Abbreviations}

- 4-MuH - 4-methylumbelliferyl heptanoate

- Ah - Aeromonas hydrophila

- Bam - $\beta$-barrel assembly machinery

- Bp-Burkholderia pseudomallei

- cPLA2 - Cytosolic phospholipase A2

- DAG - Diacylglycerol

- E. coli - Escherichia coli

- EPS - Exopolysaccharides

- FplA - Fusobacterium phospholipase autotransporter

- IAT - Inverse autotransporter

- Ig - Immunoglobulin

- IM - Inner membrane

- IP3 - Inositol 1,4,5-triphosphate

- $k_{\text {cat }}$-Substrate turnover rate

- $K_{m}-$ Michaelis constant

- LB - Lysogeny broth

- OD - Optical density

- OM - Outer membrane

- OMP - Outer membrane proteins

- PA - Phosphatidic acid

- PBS - Phosphate-bufferd saline

- PE - Phosphatidylethanolamine

- PES - Polyethersulfone

- PGN - Peptidoglycan

- PG - phosphatidylglycerol

- PI - Phosphatidylinositol

- PLA - Phospholipase A

- PIA - Phospholipase autotransporters

- PLB - Phospholipas B

- PLP - Patatin-like protein

- PlpD - Patatin-like protein D

- PM - Plasma membrane 
- POTRA - Polypeptide transport-associated

- PPlase - Peptidyl-prolyl isomerase

- PS - Phosphatidylserine

- Rs - Ralstonia solanacearum

- SEC - Size exclusion chromatography

- Sec system - General secretion system

- SRP - Signal recognition partical

- T1SS - Type one secretion system

- T2SS - Type two secretion system

- T3SS - Type three secretion system

- T4SS - Type four secretion system

- T5dSS - Type $5 d$ secretion system

- T5SS - Type five secretion system

- T6SS - Type six secretion system

- TAA - Trimeric autotransporter adhesins

- Tat system - Twin arginine translocation system

- TLC - Thin layer chromatography

- TPS - Two-partner secretion system

- YadA - Yersinia adhesin A 


\section{Contents}

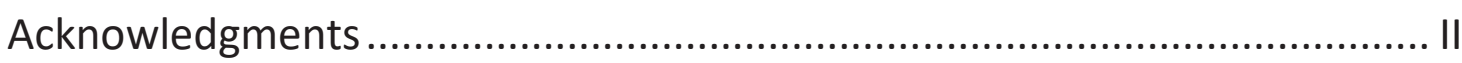

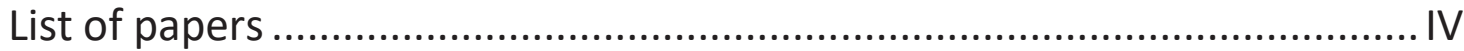

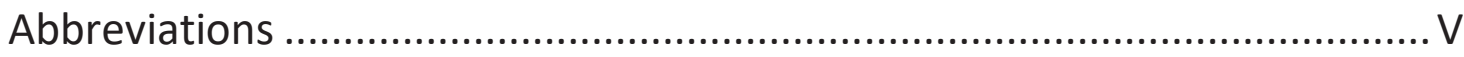

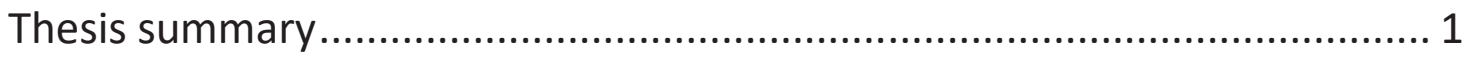

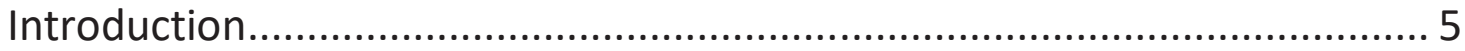

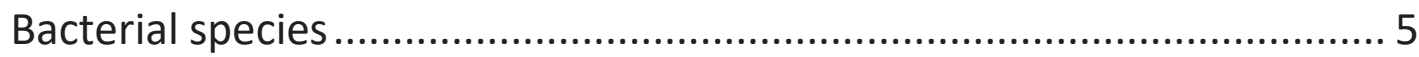

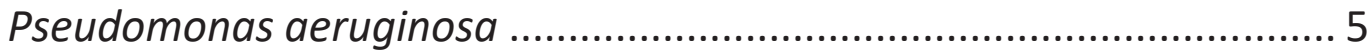

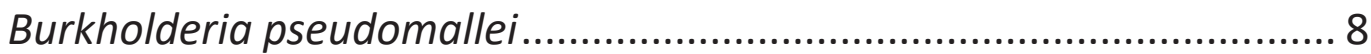

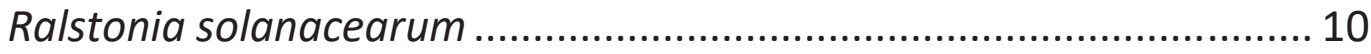

Aeromonas hydrophila .................................................................... 11

Fusobacterium nucleatum .............................................................. 14

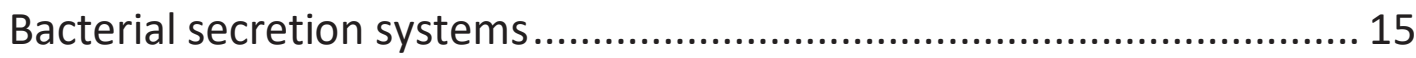

Protein secretion across the bacterial cytoplasmic membrane............... 19

Sec-mediated protein secretion ......................................................... 20

The $\beta$-barrel assembly machinery ..................................................... 22

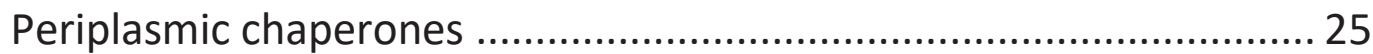

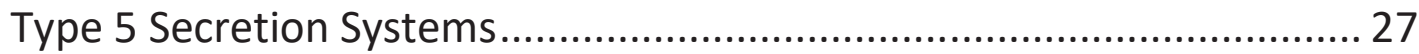

The Type 5a Secretion System: Classical autotransporters................... 30

The Type 5b Secretion System: Two-Partner Secretion Systems......... 34

The Type 5c Secretion System: Trimeric Autotransporters ................. 36

The Type 5d Secretion System ......................................................... 38

The Type 5e Secretion System: Inverse Autotransporters................... 40

Type 5d-secreted passengers....................................................................... 42

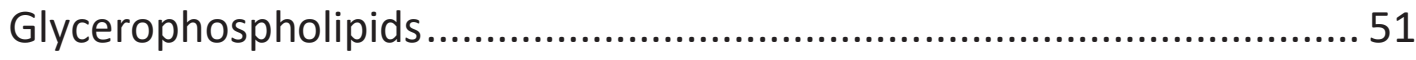

In-vivo infection model: Galleria mellonella ............................................ 54

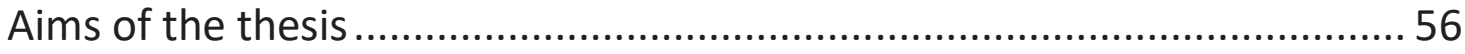




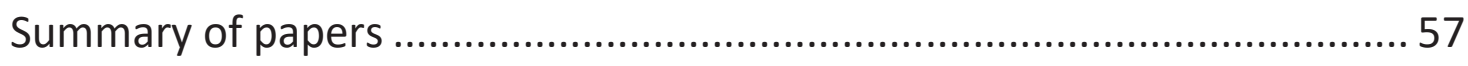

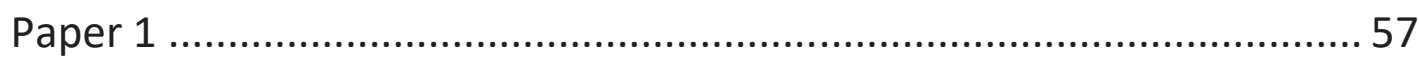

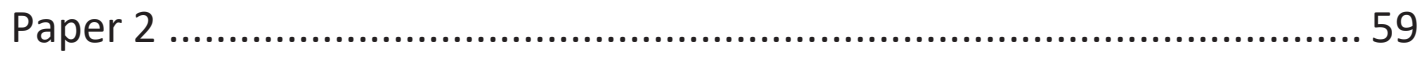

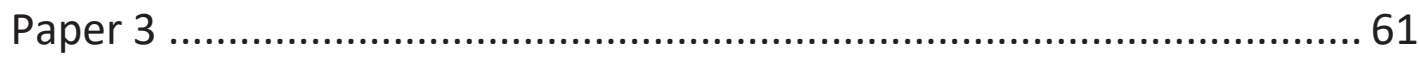

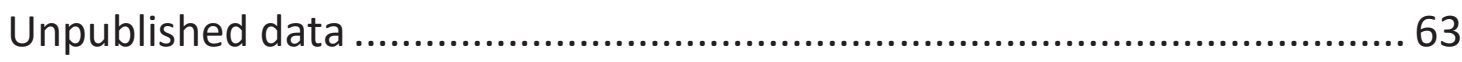

$\mathrm{PlpD}$ is non-essential for pathogenicity of $P$. aeruginosa in Galleria

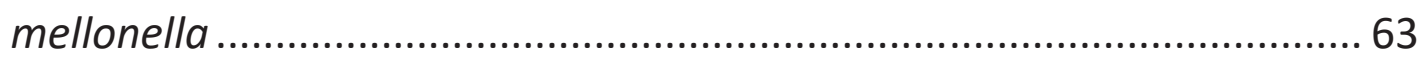

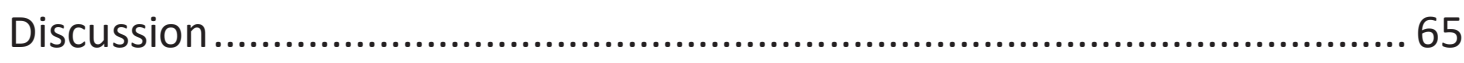

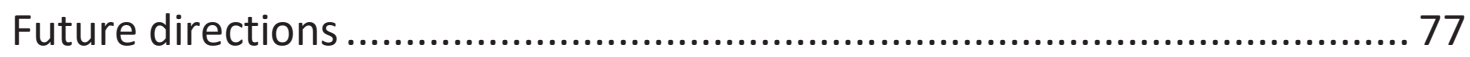

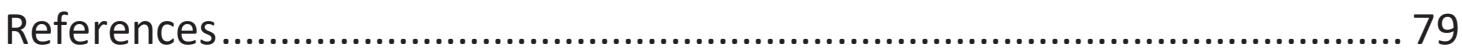




\section{Thesis summary}

Autotransporters, or type 5 secretion systems (T5SS), are widespread surface proteins of Gram-negative bacteria often associated with virulence. Autotransporters consist of an outer membrane $\beta$-barrel domain and an exported passenger. In the poorly studied type $5 d$ subclass, the passenger is a patatin-like phospholipase. The prototype of this secretion pathway is PIpD of Pseudomonas aeruginosa, an opportunistic human pathogen. Belonging to the group of acyl hydrolases, PlpD possesses phospholipase A1 (PLA1) activity and forms homodimers upon passenger secretion. Based on sequencing data, PlpD-like proteins are present in over 200 bacterial genomes.

The results presented in this thesis complement the characterization of the only two T5dSS phospholipases described in more detail so far, PlpD from Pseudomonas aeruginosa and FplA from the oral pathogen Fusobacterium nucleatum. In addition, I characterize three novel T5dSSs expressed in the fish pathogen Aeromonas hydrophila, the phytopathogen Ralstonia solanacearum and the human pathogen 
Burkholderia pseudomallei. By means of comparative analyses of the T5dSSs mentioned, we present common features shared by this group of proteins while highlighting their differences. In the case of $A$. hydrophila, we were able to solve a low-resolution structure of the respective phospholipase autotransporter (PIA) at $5 \AA$ resolution, showing high structural similarity with PIpD. Like PIpD and FplA, all tested passengers possess esterase activity, belonging to the group of acyl hydrolases which catalyze the hydrolysis of fatty acids specifically at the $s n-1$ and/or sn-2 position of phospholipids. The PIAs interact with several major lipids including phosphatidylinositol (PI), phosphatic acid, phosphatidylserine and phosphatidylglycerol. Despite the observed interaction, only FplA and BpPIA displayed hydrolytic activity towards phosphatidylethanolamine and phosphatidylserine. A common feature shared by all PIAs is the formation of higher molecular-weight complexes with two or more subunits of the same protein. Homodimer formation, as shown for PlpD, is non-essential for enzymatic activity while beneficial for thermal stability at higher temperatures. We cannot exclude however, that complex formation is 
also involved in specific substrate binding or regulation of enzymatic activity. Interestingly, we observed a correlation between the enzymatic activity and the lifestyle of the source bacteria, with a relatively low activity from extracellular pathogens and a relatively high activity from intracellular pathogens. When it comes to direct toxicity of PIAs in an in vivo model using the greater wax moth Galleria mellonella, only the two PIAs from A. hydrophila and B. pseudomallei, possessing phospholipase B activity, demonstrated any toxicity. Taken together, the higher activity of PIAs from intracellular pathogens, the apparently narrow substrate range, and the low toxicity of PIAs point towards a very specific, possibly intracellular role for these proteins in virulence. We therefore propose a role in modulating host signaling events during intracellular infection as a hypothesis for future research.

Furthermore, we could show that PIpD is dependent on the chaperone SurA for periplasmic transit and/or subsequent incorporation of PlpD into the outer membrane (OM) and that SurA is an important factor in $P$. aeruginosa assuring $\mathrm{OM}$ integrity and determining proper 
composition of the OM. Inhibition of SurA may lead to reduced fitness, dampen multidrug resistance, abolish the bacteria's ability to adhere to and invade host cells, and could simultaneously render $P$. aeruginosa accessible to various antibiotics. Thus, SurA seems to be an attractive anti-infective drug target for future research.

This thesis contributes to understanding and counteracting the pathogenesis of important bacterial pathogens, provide information that could address the pressing need for alternative therapies for emerging antibiotic-resistant infections, and supply detailed molecular information about how bacteria secrete proteins to the extracellular milieu. 


\section{Introduction}

\section{Bacterial species}

Most bacteria are microscopic, single-celled, ubiquitous organisms that thrive in diverse environments. These prokaryotic organisms are important factors in every aspect of life, being at the same time commensals or essential symbionts, conferring beneficial traits to their environment and/or interaction partners, while also being the causative agent of variety of infectious diseases, both in fauna and flora. Illuminating the factors and mechanisms behind bacterial virulence in the individual diseases will allow us to develop protective as well as therapeutic treatments thereby reducing the collateral damage on humans and environment.

Pseudomonas aeruginosa

Pseudomonas aeruginosa is a member of the $\gamma$-Proteobacteria class of Gram-negative bacteria. It is a rod-shaped, monoflagellated bacterium belonging to the bacterial family Pseudomonadaceae. It occupies a 
broad range of natural habitats, commonly found in soil as well as water, typically attached to some surface or substrate as biofilm. Next to this sessile lifeform, it is also found in a unicellular, planktonic form, actively swimming by means of its flagellum (1). Pseudomonas aeruginosa is classified as an opportunistic pathogen, thus causing disease infrequently in healthy hosts but being a major problem in immunocompromised ones. The combination of having simple nutritional requirements, an optimum temperature for growth of $37^{\circ} \mathrm{C}$, although it grows up to a temperature of $42^{\circ} \mathrm{C}$, and an intrinsic resistance to a wide range of commonly used antibiotics results in Pseudomonas aeruginosa being one of the major players in nosocomial infections, causing septicemia, urinary tract infections, pneumonia, chronic lung infections, endocarditis, dermatitis and osteochondritis (2-6). Pseudomonas infection can roughly be subdivided in three stages: attachment and colonization; local invasion; and disseminated systemic disease. Tissue colonization usually precedes host invasion, depending on a range of bacterial adhesion proteins binding to specific receptor proteins of the host (1). 
Colonization in some cases depends on the active degradation of the target tissue by means of certain proteases or existing tissue damage in order to reveal the respective receptors. The latter was termed opportunistic adhesion and may play an important role in urinary and respiratory tract infections caused by this pathogen (1). Upon tissue colonization, Pseudomonas aeruginosa employs extracellular proteases and toxins that actively disrupt host defenses and enable the bacterium to invade host tissue and avoid immune defenses. The proteases elastase and alkaline protease are directly associated to Pseudomonas virulence. Elastase cleaves collagen, IgG, IgA and complement (7), and lyses fibronectin thus exposing receptors for bacterial attachment on the mucosa of the lung $(8,9)$. Alkaline protease lyses fibrin and interferes with fibrin formation. Next to those two enzymes, Pseudomonas aeruginosa produces at least three other extracellular proteins involved in invasion, a pore-forming cytotoxin and two hemolysins, a phospholipase as well as a lecithinase (10). Disseminated systemic disease, although the mode of action being not 
entirely clear yet, is probably mediated by the same factors also responsible for local colonization and invasion.

\section{Burkholderia pseudomallei}

Burkholderia pseudomallei are Gram-negative bacteria belonging to the class of $\beta$-Proteobacteria. It is an aerobic, flagellated, soil-dwelling bacterium endemic in Southeast Asia and Northern Australia. Although most species of the genus Burkholderia are plant pathogens, Burkholderia pseudomallei can cause disease in humans and is the causative agent for melioidosis. Individuals can get infected by cutaneous inoculation as well as inhalation and aspiration. From this local site of infection, Burkholderia pseudomallei can disseminate throughout the body, infecting organs such as the lung, liver or spleen, leading to pneumonia and in the worst case to septic shock. Treatment is difficult due to the bacterium's intrinsic resistance to a wide range of commonly used antibiotics, the absence of a vaccine and its facultative intracellular lifestyle, resulting in a high overall mortality (11-13). Burkholderia pseudomallei can invade a variety of cells, both 
phagocytic and non-phagocytic. Although Burkholderia shares common virulence factors with other Gram-negative pathogens, the specific virulence factors involved during certain steps of host cell invasion remain speculative. Burkholderia pseudomallei has three gene clusters encoding for type three secretion systems (T3SS). The best characterized being the T3SS3, which shares high homology with the Inv/Mxi-Spa secretion systems of Salmonella spp. and Shigella flexneri, respectively $(14,15)$. T3SSs are multiprotein machineries spanning the bacterial envelope, tasked with the secretion of bacterial effector proteins from their point of synthesis in the bacterial cytosol, across the extracellular space, directly into the host cell. Next to their role in host cell invasion, T3SSs often play an essential role in escaping endocytic vacuoles into the cytosol (16). In B. pseudomallei, the T3SS3 is involved in the escape from endocytic vesicles (14) and required for full virulence in murine models of melioidosis (17). In addition, $B$. pseudomallei produces an extracellular capsular polysaccharide (18), which probably acts as a barrier and thus reducing complement receptor-3b deposition on the bacterial surface (19), as well as other 
putative virulence factors, like a secreted haemolysin, lipases and proteases (20).

\section{Ralstonia solanacearum}

Ralstonia solanacearum is a member of the $\beta$-Proteobacteria class of Gram-negative bacteria. It is a soil-borne, rod-shaped and motile plant pathogen that causes bacterial wilt on a wide range of host plants comprising more than 200 species in at least 50 families worldwide (21). When the phytopathogen encounters a susceptible host, it invades the plant roots and colonizes the root cortex followed by invasion of the xylem vessels and subsequent spread to aerial parts of the plant through the vascular system (22). The two main factors determining virulence are the pathogen's T3SS and its high molecular mass exopolysaccharides (EPS). The T3SS allows direct transfer of substrates from the bacterial cytosol into the host cells to favor infection by influencing host behavior. In the absence of a functioning T3SS R. solanacearum is rendered non-pathogenic $(23,24)$. In addition to its T3SS, $R$. solanacearum also secretes a heterogenous mixture of 
EPS which are involved both in root infection as well as vascular colonization. EPS contributes in the constriction and finally the occlusion of the plant's xylem vessels causing wilting symptoms (23, 25). Although being the most important factors in $R$. solanacearum pathogenicity, the pathogen produces multiple other factors contributing to invasion, colonization or disease symptoms (26). These include type 2 secreted plant cell degrading enzymes, motility or attachment appendages, aerotaxis transducers, cellulases and pectinases (23). Furthermore, $R$. solanacearum has several other secretion systems, including T1SS, T4SS, T5SS and T6SS, often involved in infection processes in other Gram-negative bacteria and that might have a yet unknown impact on pathogenicity or possibly complement the actions of T2SS and T3SS secreted proteins.

Aeromonas hydrophila

Aeromonas hydrophila is a member of the $\mathrm{y}$-Proteobacteria class of Gram-negative bacteria. It is a rod-shaped, monoflagellated, ubiquitous bacterium belonging to the bacterial family 
Aeromonadaceae. This facultative anaerobic chemoorganoheterotroph has an optimal temperature for growth between $22^{\circ} \mathrm{C}$ to $28^{\circ} \mathrm{C}$ and can infect insects, amphibians, birds and reptiles while being a disease-causing pathogen of fish and human (2730). A. hydrophila is a facultative intracellular pathogen (31) which causes hemorrhagic septicemia in fish that often leads to an elevated mortality and major economic losses in aquaculture (32). It is also the etiological agent of disease in both immunocompetent and immunocompromised human patients, causing effects ranging from relatively mild conditions such as acute gastroenteritis to lifethreatening septicemia, necrotizing fasciitis and myonecrosis $(27,33$, 34). Once A. hydrophila in its motile, planktonic form is in proximity of a susceptible host, it starts host cell colonization and promotes production of virulence factors necessary for establishment of infection, including adhesins, cytotoxins, hemolysins and proteases $(33,35,36)$. The cytotoxic enterotoxin Act, a pore-forming toxin, is one of the main virulence factors in $A$. hydrophila and responsible for hemolytic, cytotoxic and enterotoxic activities during host cell 
infection and immune evasion $(37,38)$. In addition, extracellular lipases can be found in A. hydrophila, among them the lipases Alt and Ast, cytotonic enterotoxins also found in A. dhakensis SSU, where they play an important role during pathogenicity (39-41). Although their role during infection in $A$. hydrophila remains speculative, their lipolytic activity may generate free fatty acids which interfere with host cell signaling or have cytotoxic activities as in the case of phospholipase C in Aeromonas spp. Serogroup 0:34 (42). Another possible function might be the provision of nutrients or the complementation of other virulence factors. Next to extracellular virulence factors, the presence of a functional type 3 and type 6 secretion system, which play a critical role in the virulence of many Gram-negative bacteria, have been identified in $A$. hydrophila. Insertional inactivation of essential genes in the T3SS gene cluster as well as deletion of virulence-associated secretion genes essential for a functional T6SS led to decreased cytotoxicity, increased phagocytosis and reduce virulence $(43,44)$. More virulence factors of $A$. hydrophila are determined continuously, yet the exact function and the potential 
role of an array of extracellular proteins as well as secretion systems known to date remain open for speculation.

\section{Fusobacterium nucleatum}

Fusobacterium nucleatum is a Gram-negative bacterium of the family Fusobacteriaceae in the phylum Fusobacteria. This rod-shaped, usually non-motile, anaerobic bacterium is commonly found in the mouth and other mucosal sites of humans and other mammals (45). It is part of the natural microbiota of these healthy tissues but has also been repeatedly isolated from clinical samples. As an opportunistic pathogen, $F$. nucleatum plays integral and beneficial roles in biofilms that contribute to both periodontal health and disease. During the progression of a disease, it interacts closely, or even cooperatively, with other oral microorganisms to establish infection and to evade the inflammatory response of the host $(46,47)$. In order to do so, it encodes several adhesins for interspecies interaction, including Fap2, RadD, and Aid1 (48-50). The two outer membrane proteins (OMPs) Fap2 and RadD, belonging to the group of T5SSs, share the ability to 
induce cell death in Jurkat cells, possibly through the activation of the host cell's apoptotic pathways. Deletion of Fap2 and RadD resulted in a $95 \%$ decrease in cell death induction in Jurkat cells compared to wild type cells. Induction of cell death by cell-free $F$. nucleatum membrane preparations indicates that the mechanism is a passive one, independent of any active process or effector protein transfer (48). Fusobacterium also binds to a variety of mammalian cells and invades epithelial and endothelial cells (51-53), being implicated in a wide spectrum of infections and abscesses, as well as colorectal cancer. Independent of the respective host cell, adherence and invasion are essential mechanisms for colonization, dissemination, evasion of host defense, and induction of host responses.

\section{Bacterial secretion systems}

A bacterial cell is faced with a variety of unpredictable, ever changing and often hostile environments. In order to survive, bacteria have evolved a complex bacterial envelope to protect themselves from the extracellular environment as well as to maintain the structural 
integrity of the bacterial cell while allowing selective transport between the cytoplasm and the extracellular space in order for nutrients to be taken up, waste to be disposed of, signals to be transduced and proteins to be presented on or release from the cell surface. Especially for pathogenic bacteria, the ability to present proteins on the cell surface as well as to secrete proteins into the extracellular space or directly into the host cell is an essential trait necessary to establish and promote host cell infection.

The bacterial envelope of Gram-negative bacteria comprises two membranes, the inner membrane (IM) and the outer membrane (OM), separated by the periplasmic space containing the peptidoglycan cell wall. Whereas the IM is composed of diglyceride phospho- and glycolipids, the OM is an asymmetric assembly of phospholipids in the inner leaflet and lipopolysaccharides in the outer leaflet (Fig.1). For proteins to cross this barrier without compromising the integrity of the bacterial envelope, bacteria utilize a multitude of bacterial secretion systems spanning either the OM or the complete bacterial envelope. The transport of bacterial proteins from the cytosol into the 
environment or host cells is a process known as protein secretion. Secretion systems in Gram-negative bacteria can be subdivided into type 1 through type 9 secretion systems $(54,55)$. Some of these secretion systems are widespread in Gram-negative bacteria, responsible for the secretion of a large variety of proteins, whereas other secretion systems are only found in a small subset of Gramnegative bacteria, sometimes being responsible for the specific secretion of a single protein. The difference in the number of secreted proteins is reflected in the complexity of the different secretion systems, with some secretion system consisting of only a single polypeptide chain, like some subclasses of the T5SS $(56,57)$, and others being macromolecular complexes consisting of a variety of different proteins with copy number ranging from 1 to more than 100 (58), like the T3SSs (16) 


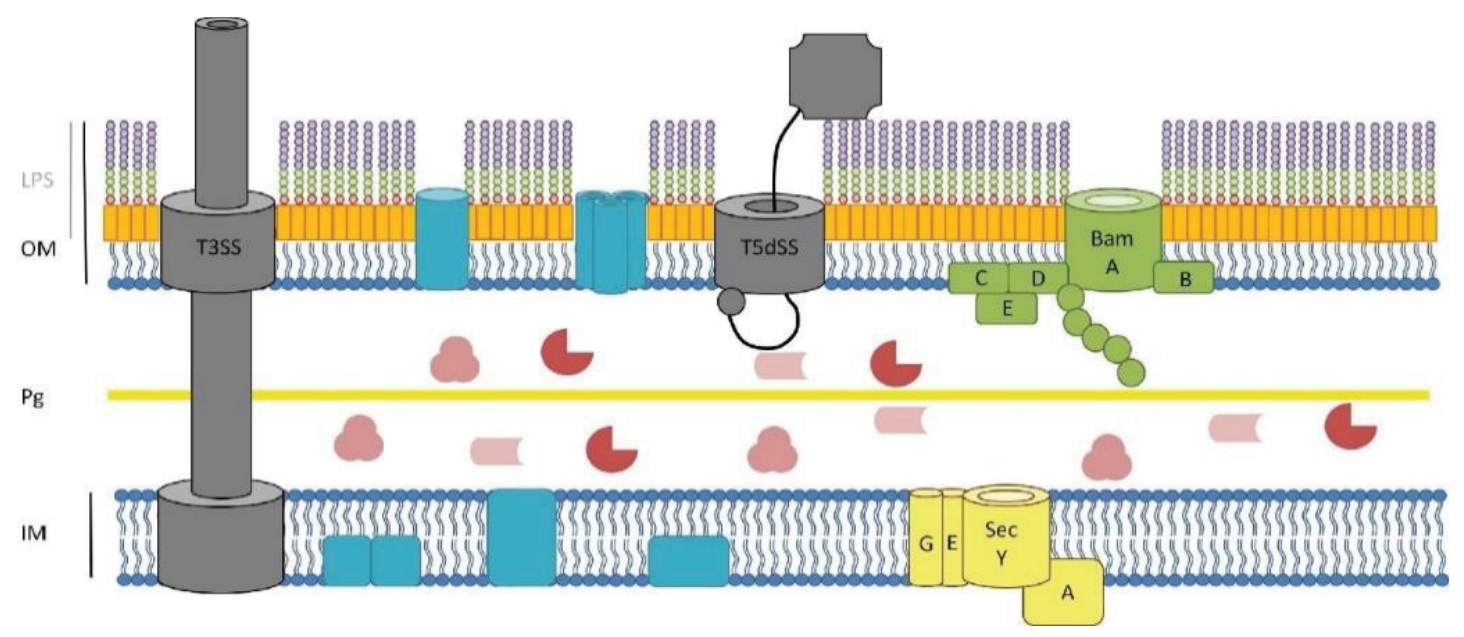

Figure.1: General structure of a bacterial envelope in Gram-negative bacteria. The bacterial envelope is composed of the inner membrane (IM) and the outer membrane $(\mathrm{OM})$, separated by the periplasmic space containing the peptidoglycan cell wall (PGN). Whereas the IM is a symmetric lipid bilayer composed of phospholipids and integral membrane proteins, the $\mathrm{OM}$ is an asymmetric bilayer containing phospholipids in its inner leaflet and lipopolysaccharides (LPS) in its outer leaflet. The periplasm is an aqueous compartment between the IM and the OM where the peptidoglycan cell wall resides. A variety of proteins are associated with the bacterial cell envelope, including chaperones, protease and other periplasmic enzymes (colored in shades of red), porins and integral membrane proteins (colored in shades of blue), bacterial secretion systems (colored in shades of grey) and other membrane specific protein machineries (colored in shades of green and yellow). 
Protein secretion across the bacterial cytoplasmic membrane

The cytoplasmic membrane is a phospholipid bilayer membrane which constitutes the permeability barrier between the aqueous cytosol and the periplasm. This membrane is a barrier for free ion diffusion in either direction, allowing for a controlled ionic composition of the cytosol and creating the platform for membrane-spanning proteins which can use the ionic gradient between those two compartments for energy consuming processes (59). It also prevents the uncontrolled translocation of proteins and other macromolecules from their location of synthesis in the cytoplasm across the membrane. Proteins destined for incorporation into the membrane or for secretion are selectively targeted to one of two major translocation machineries at the cytoplasmic membrane, the general secretion (Sec) system (60) or the twin arginine translocation (Tat) system (61). These two systems allow the secretion of proteins across the cytoplasmic membrane in a controlled manner, without compromising structural or functional properties. The major difference between these two secretion systems is that the Sec system transports unfolded proteins into the periplasm, 
whereas the Tat system is responsible for the translocation of folded proteins.

\section{Sec-mediated protein secretion}

The Sec system is involved in the post-translational translocation of precursor proteins across the cytoplasmic membrane as well as the cotranslational targeting of integral membrane proteins to the cytoplasmic membrane(62). The core of the multi-protein Sec machinery is formed by the translocase. Translocase is the complex formed by three integral membrane proteins, SecY, SecE and SecG, as well as the peripherally bound ATPase SecA, facing the cytosol (6365)(Fig.1). Proteins destined for secretion have an N-terminal signal sequence. Depending on the protein, these signal sequences can vary in length from 18 to approximately 30 amino acid residues (62), with the exception of certain members of the autotransporter family, which have an extended signal sequence up to 47 amino acid residues (66). In a post-translational pathway, the signal sequence is subsequently recognized by the cytosolic export chaperone $\operatorname{Sec} B$, forming a 
substrate-chaperone complex. This complex is, in turn, recognized by the ATPase SecA (67). Upon interaction with the protein-chaperone complex, SecA binds ATP at one of its two ATP binding sites which drives the insertion of certain SecA domains as well as the $\mathrm{N}$-terminus of the precursor protein across the membrane $(68,69)$. After insertion of the precursor protein, ATP hydrolysis triggers the release of the inserted protein and SecA retracts from its membrane inserted state as a result of the binding of ATP at the second ATP-binding site on the SecA molecule (68). Repeated cycles of ATP binding and hydrolysis results in the stepwise translocation of the target protein across the cytoplasmic membrane.

Most of the integral membrane proteins which reside in the cytoplasmic membrane have $\alpha$-helical membrane spanning regions. For them to function properly, they must be directed to the correct membrane, inserted and folded into the correct conformation. To this end, the protein-conducting channel SecYEG (70) of the Sec-translocon is essential for translocation and membrane protein insertion. Similar to the precursor proteins, SecA uses repetitive ATP hydrolyses to push 
the inserting polypeptide chain through the SecYEG channel. In contrast to Sec dependent translocation of precursor proteins, targeting of integral membrane proteins is co-translational. The signal recognition particle (SRP) interacts with a membrane protein as it emerges from the ribosome at the membrane surface (71). The SRPribosome-protein complex is subsequently targeted to its receptor FtsY, which is located at the membrane. Transfer of the protein to the translocation channel is GTP-dependent and facilitated through direct interaction of FtsY and SecY (72).

The $\beta$-barrel assembly machinery

The bacterial $\mathrm{OM}$ is an asymmetrical bilayer which functions as a selective permeability barrier and is an essential organelle of Gramnegative bacteria. The inner leaflet of this bilayer consists of phospholipids whereas the outer leaflet consists mainly of lipopolysaccharides. Integral OMPs allow the controlled interaction with the environment. Most of these integral membrane proteins have multiple amphipathic $\beta$-strands that fold into a characteristic $\beta$-barrel 
structure with a hydrophilic interior and hydrophobic residues pointing outward, facing the membrane lipids (73). Depending on the protein, the $\beta$-barrel functions as a membrane anchor or as a pore forming channel. Although it is known that most OMPs cross the IM via the Sec pathway and are escorted to the inner leaflet of the OM by periplasmic chaperones, with SurA being the primary chaperone responsible for the periplasmic transit (74)(see below), the exact mechanism of OM insertion remains open for debate. However, it is generally accepted that OMP membrane integration is catalyzed by the $\beta$-barrel assembly machinery (Bam), previously known as Omp85 and YaeT complex (75-77) in reference to the old names for BamA.

The $200-\mathrm{kDa}$ Bam complex is formed through the interaction of five proteins, the integral OMP BamA, containing 5 polypeptide transportassociated (POTRA) domains, and four lipoproteins (BamB, BamC, BamD and BamE) (75-77) binding to the POTRA domains, forming a ring like structure at the inner leaflet of the OM (Fig.1). Although the molecular structure of the Bam complex has been solved (78-80), the mechanism by which $\beta$-barrel proteins are inserted into the OM 
remains poorly understood. The threading model suggests that the rotation of the ring-like structure formed by BamB through BamE results in the opening of a gap between the first and last $\beta$-strand of the BamA $\beta$-barrel thereby promoting the insertion of the nascent OMP $(78,79)$. In this model, the $\beta$-strands of unfolded OMPs are sequentially inserted into the OM through the lateral opening in BamA, creating a hybrid-barrel during the assembly process, which is subsequently separated when folding is complete $(81,82)$. The assisted model posits that the BamA $\beta$-barrel causes membrane disruption of the adjacent membrane allowing the insertion of OMPs at the locally deformed lipid bilayer $(82,83)$. The most recent model postulates that the BamA $\beta$-barrel forms two dissimilar interfaces with the assembly intermediate creating an 'asymmetric hybrid barrel', which promotes the integration of partially folded $\beta$-barrel from the periplasm in the OM by a swing mechanism (84), rather than the sequential insertion suggested in the threading model. 


\section{Periplasmic chaperones}

The periplasm is an aqueous space separating the inner and the outer membrane. In contrast to the IM, nutrients usually pass the OM by passive diffusion through a class of OMPs called porins (85). Due to this passive influx, the periplasm shares many aspects with the external environment. It is in general oxidizing, contains salt, has $\mathrm{pH}$ levels similar to the outside of the cell and does not contain ATP (86). In addition, a layer of peptidoglycan is located in the periplasm. For OMPs to reach the OM after Sec-dependent passage across the IM, specific helper proteins, periplasmic chaperones, guide these proteins to their final destination, prevent undesired protein-protein interactions within the periplasm, protect them from degradation and assist in the correct folding of the protein.

Most periplasmic chaperones that have been identified can be divided roughly into two groups with a certain amount of functional overlap. The first group conveys highly specific steric information essential for the correct folding of the respective polypeptide chains to which these chaperones are reversibly attached (87) while protecting their bound 
substrate from aggregation as well as degradation and targets them to their destined location. Substrates dependent on this first group of proteins, like the lipase-specific foldase (Lif) from Burkholderia glumae (88), do not fold correctly into their native structures without the help of steric chaperones. The second group binds to a variety of different unfolded proteins, assisting them in the correct folding and protecting them from degradation. The second group includes the chaperones SurA, Skp and DegP, and function as chaperones in the OMP targeting pathway (74). SurA belongs to the peptidyl-prolyl isomerase (PPlase) family which also possesses PPlase-independent chaperone activity (89) and is the primary chaperone responsible for the periplasmic transit of the majority of OMPs to the Bam complex (74). Skp/DegP are functionally redundant proteins which function in a parallel pathway to SurA, rescuing OMPS that are not covered by the SurA pathway. Deletion of Skp and DegP has no effect on OM composition while SurA depletion results in a significant decrease in OMP density. In a SurA depletion mutant however, Skp and DegP take over the functional role of SurA. Thus, Skp/DegP play only a minor role under normal growth 
conditions but is greatly upregulated upon stress induction or in the absence of SurA (74). Although of marginal importance under optimal growth conditions, DegP is essential for growth at higher temperatures due the protein`s ability to switch from chaperone to proteolytic activity and its role in protein quality control in the bacterial envelope (90).

\section{Type 5 Secretion Systems}

Probably the simplest as well as most widespread secretion system in Gram-negative bacteria is the T5SS. Depending on their structural features as well as their domain organization, T5SSs can be subdivided into the subclasses type 5a through type 5e (Fig.2). The different subclasses of T5SSs share some common features. They are proteins consisting of a single polypeptide chain with two functional regions. The exception is the T5bSS which consists of two separate polypeptide chains with one functional domain each. The first domain is the so called "translocator". The translocator domain forms a 12 or 16 stranded $\beta$-barrel which is incorporated into the OM. In the case of 
type $5 b$ and type $5 d$, two or one POTRA domains are attached to the $\beta$-barrel, protruding into the periplasmatic space. The function of the OM-spanning translocator domain is the secretion of the second domain from the periplasmic space to the cell surface. The second domain being dependent on the incorporation of the translocator domain into the OM prior to its secretion led to the name "passenger domain". The translocator and the passenger domain are connected via a short linker sequence. They all possess an $\mathrm{N}$-terminal signal sequence, which is recognized by the Sec-machinery and which guarantees targeted passage of the unfolded T5SS across the IM. T5SSS depend on periplasmic chaperones for the transit through the periplasm and on the Bam complex for integration of the $\beta$-barrel domain into the OM (91-93). Like all active transport across membranes, also the translocation of the passenger domains is energy dependent. The source providing this energy is still open for debate since there is no obvious energy source at hand. Chemical energy such as ATP is absent in the periplasm and no proton or other ionic gradients exist across the $\mathrm{OM}$ to drive secretion. The lack of an energy 
source led to the assumption of type 5 secretion being completely autonomous (94). Due to the presumably self-sufficient secretion of the T5SS, this group of proteins is also known as "autotransporters". Some models posit that the energy, necessary for passenger translocation comes from the intrinsic folding capacity of the respective autotransporter itself (95-97), that translocation is influenced by electrostatic interactions between the negatively charged passenger with either membrane proteins or lipids (98) or that energy is transduced from the cytoplasm by an IM protein spanning the periplasmic space. 


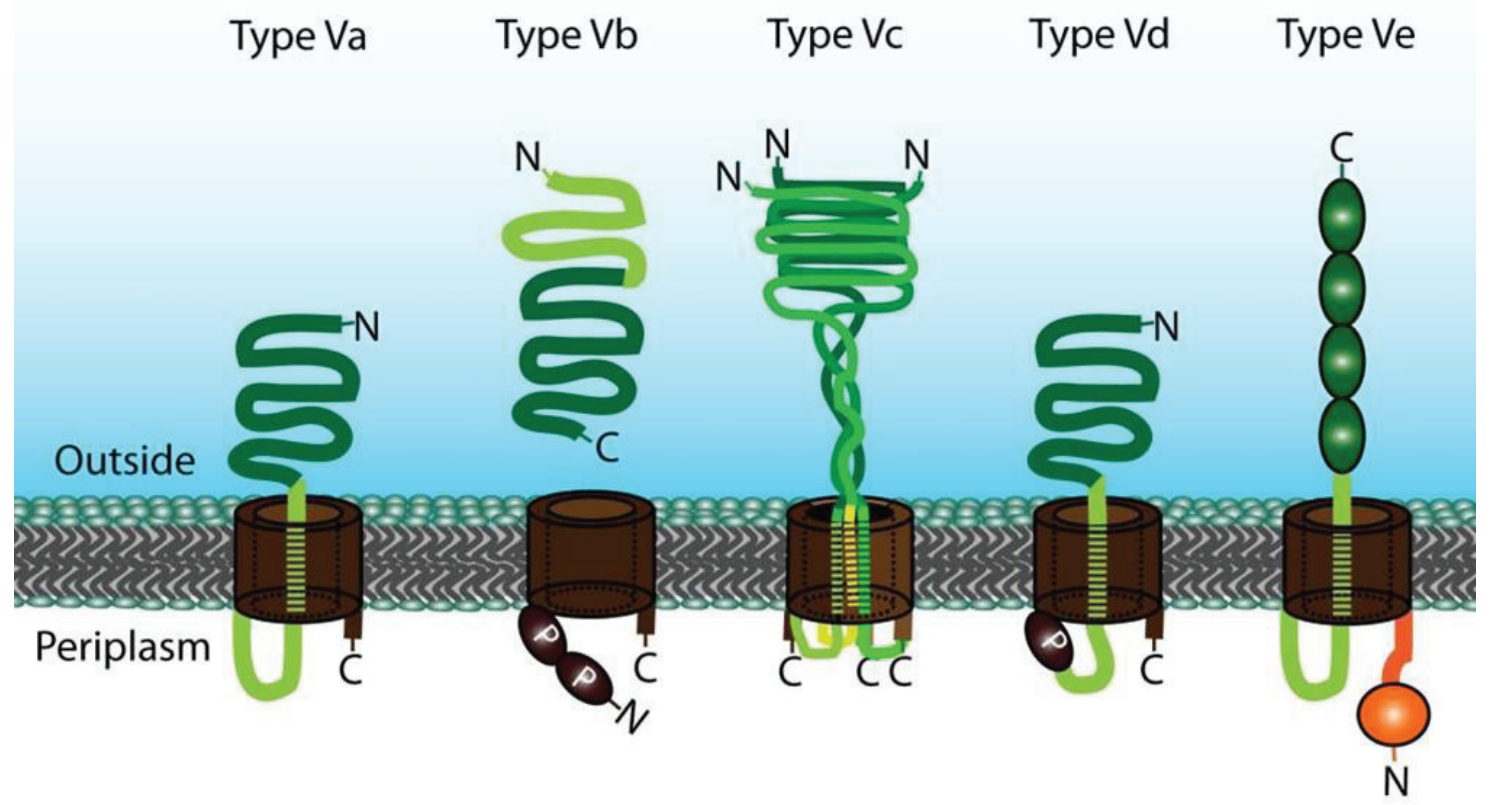

Figure 2. "Topology models of the different type $\mathbf{V}$ secretion systems. The translocation domain is displayed in brown, linker/Tps regions in light green, passenger domains in dark green and periplasmic domains in orange. POTRA domains are labelled (P). For clarification of the topology, $\mathrm{N}$ - and C-termini are indicated". Figure adapted from Leo et al., Philos Trans R Soc Lond, Biol Sci. 367:10881101. (57). [CC-By: https://creativecommons.org/licenses/by/4.0/].

The Type 5a Secretion System: Classical autotransporters

Type 5a secretion systems are a family of monomeric autotransporters, also called classical autotransporters. They were the first described and most extensively studied autotransporters, both in 
regard to function and structure. Among them, the extracellular IgA protease from Neisseria meningitidis, which is correlated specifically to pathogenesis of neisserial infections (99) and the first protein for which the type 5 secretion model was described (100). As early as 1987, Pohlner et al. (100) described an IgA protease precursor protein with three functional regions, the amino-terminal leader sequence to initiate the IM transit, the protease and the carboxy-terminal helper domain required for extracellular secretion. The authors proposed a model where the helper domain serves as a pore for passage of the protease domain across the OM. Since this first description, many proteins with the same secretion pathway have been identified, as what we now call autotransporters. They all share the same primary structure as described for IgA, consisting of three functional domains, an $\mathrm{N}$-terminal signal sequence (leader sequence) for Sec-dependent IM passage, the passenger domain conferring the autotransporter's specific function and a C-terminal translocator (helper-domain) domain facilitating the secretion of the passenger domain across the OM. Although similar in structure, T5aSSs have diverse functions and 
are known virulence factors in a wide range of different bacteria. They can act as extracellular proteases, lipases or adhesins for a wide range of substrates $(101,102)$.

T5aSSs are single polypeptide chains harboring all three functional domains. Sec dependent passage across the IM is similar compared to other Sec-dependent proteins, although a subset of the T5aSSs are synthesized with an unusually long signal peptide distinguished by a unique $\mathrm{N}$-terminal sequence motif, among them the Escherichia coli hemoglobin protease ( $\mathrm{Hbp})(103)$ and serine protease EspP (104). Hbp interacts early during biogenesis with SRP and the Sec translocon while being SecB-independent, although SecB can rescue an SRP depletion phenotype to a certain degree (103). Modification of the specific sequence motif of Esp does not affect IM transit but results in misfolding of the protein in the periplasm and stalled translocation of the passenger domain across the OM (104). Thus, the extended signal sequences often function as targeting signals for the co-translational SRP/Sec-dependent translocation pathway (103) but also prevent misfolding of the nascent protein in the periplasm (104). The proposed 
mechanism behind the prevention of misfolding is that the specific signal motif interacts with the Sec machinery, stalling the release of the nascent protein into the periplasm and thereby prolonging the interaction long enough to promote binding of periplasmic chaperones before misfolding can occur (104). The interaction of the periplasmic chaperones SurA, Skp, the chaperone/protease DegP and the cis/trans-prolyl isomerase FkpA with unfolded Esp $(91,105,106)$ as well as SurA with $\mathrm{Hbp}(92)$ has been shown.

The exact mechanism for insertion of autotransporters into the OM is still unclear but is facilitated by the Bam complex. EspP and Hbp interact directly with BamA $(91,92)$, which recognizes a specific Cterminal sequence motif in $\beta$-barrel proteins (107). Next to $\beta$-barrel insertion, BamA has also been implicated in passenger secretion of EspP, a theory supported by the fact that certain members of the Omp85 superfamily catalyze secretion reactions (108). Following $\beta$ barrel insertion, the passenger domain is translocated C-terminus first, forming a hair-pin structure which extends to the extracellular space while the $\mathrm{N}$-terminus remains in the interior of the $\beta$-barrel and the 
adjacent periplasm. Presumably, the intrinsic folding energy of the surface exposed segments in a sequential order is enough to drive export of the rest of the protein as shown for Pertactin, Hbp and EspP (109-111). Among type 5a autotransporters are many autocatalytic proteases that cleave off the passenger domain and release it after OM transit (57). In the case of EspP, the autoproteolytic reaction occurs inside the $\beta$-barrel, dependent on two conserved residues, an aspartate and an asparagine, forming a catalytic dyad that mediates self-cleavage through cyclization of the asparagine (112).

The Type 5b Secretion System: Two-Partner Secretion Systems

In contrast to the classical autotransporters, the passenger and translocator domain of T5bSS are translated as two separate polypeptide chains, respectively referred to as TpsA and TpsB family members (113). The expression of the genes coding for TpsA and TpsB into separate polypeptide chains led to the alternative name TwoPartner Secretion System (TPSS) for T5bSSs. In many cases the tps loci are encoded in a single operon (113), with tpsB usually upstream of its 
cognate passenger tpsA (57). However, some operons encode for more than one TpsA family member next to a single TpsB family member, as demonstrated by LspB-dependent secretion of the passengers LspA1 and LspA2 in Haemophilus ducreyi (114) and FhaCdependent secretion of the passengers FhaB and FhaS in Bordetella bronchiseptica (115). To complicate matters, some TPS systems require additional proteins to be fully functional, like in the case of the adhesins HMW1 and HMW2 of Haemophilus influenza, which require both their cognate TpsB transporters HMW1B and HMW2B as well as the glycotransferases HMW1C and HMW2C (116-118) to prevent proteolytic degradation.

As for classical autotransporters, TPS systems depend on the Sec machinery for IM transit. Once in the periplasm, periplasmic chaperones facilitate the transit to the OM while preventing misfolding and proteolytic degradation of the nascent protein, as seen in the case of DegP-dependent transit and targeting of Fha to FhaC in Bordetella pertussis (119). The mechanism of TpsA secretion across the $\mathrm{OM}$ is assumed to be similar to the classical autotransport. TpsB 
proteins form $\beta$-barrels in the OM composed of 16 anti-parallel $\beta$ strands. This pore forming $\beta$-barrel is connected to two periplasmic POTRA domains. The defining feature of proteins belonging to the TpsA family is a conserved, approximately 250 residue long $\mathrm{N}$-terminal domain essential for secretion, designated the TPS domain (120). The TPS domain directly interacts with the POTRA domains connected to TpsB (120), initiating the translocation of TpsA. The current model of passenger secretion suggests that TpsA remains associated with the POTRA domain connected to TpsB after recognition of the TPS domain while the rest of TpsA is translocated through the $\beta$-barrel via initial hairpin formation (121). The passenger is subsequently released into the extracellular space where it may or may not stay attached to the bacterial surface.

The Type 5c Secretion System: Trimeric Autotransporters

Type $5 c$ secretion systems are a family of trimeric autotransporters. The individual subunits consist of the same functional domains as classical autotransporters, with a C-terminal translocator domain and 
an $\mathrm{N}$-terminal passenger domain, but are obligate homotrimers with the translocator domain from each subunit forming the OM $\beta$-barrel, composed of 12 anti-parallel $\beta$-strands $(122,123)$. Trimeric autotransporters are important virulence factors in a broad range of Gram-negative bacteria, mediating adhesion to extracellular surfaces, both of biotic and abiotic nature. Especially adhesion to host cells is an essential step towards host cell colonization during the infection process. In contrast to monomeric autotransporters, all trimeric autotransporters studied to this point are adhesins which stay attached to the OM-anchor and do not harbor enzymatic activities (57). These trimeric autotransporter adhesins (TAAs) share a basic architecture, with a coiled-coil stalk protruding from the cell surface and an N-terminal, globular head domain. While the head and stalk domains are diverse, the membrane anchor is homologous throughout the TAAs (123). In complex adhesins, head and stalk domains may alternate several times before reaching the OM-anchor $(124,125)$.

As classical autotransporters, trimeric autotransporters have an $\mathrm{N}$ terminal signal sequence for Sec-dependent translocation across the 
IM and depend on chaperones for periplasmic transit. The periplasmic chaperone/protease DegP is in addition responsible for proteolytic degradation of erroneous proteins, as demonstrated in the case of the Yersinia adhesin A (YadA) from enteropathogenic Yersiniae (126). Trimeric autotransporters harbor a C-terminal sequence motif for specific recognition by the Bam complex for subsequent trimerization and $\beta$-barrel insertion in the OM (127). Autotransport of the passenger domains of all three polypeptide chains happens in concert via hairpin formation and stepwise transport through the translocator pore powered by the sequential folding of domains exposed to exterior (57, 128). The passenger is not released after translocation across the OM but stays linked to the respective membrane anchor, protruding from the cell surface.

The Type 5d Secretion System

Type $5 d$ secretion systems share the same domain organization with classical autotransporters, consisting of an $\mathrm{N}$-terminal signal sequence for Sec-dependent IM transit, a passenger domain and a C-terminal 
translocator domain. Although there is no structural evidence to date, the translocator domain displays characteristics of TpsB proteins with a single POTRA domain preceding the 16 -stranded $\beta$-barrel domain. Whereas the POTRA domains connected to TpsB directly interact with the TPS domain of TpsA, initiating the translocation of TpsA, the exact role of the single POTRA domain present in T5dSSs remains speculative. It might interact with yet unknown proteins necessary for protein OM insertion, like proteins of the Bam complex, facilitate the autotransport directly or, although less likely, could be an artifact, provided that T5dSSs might be an evolutionary intermediate between T5aSSs and T5bSSs. The idea of an evolutionary intermediate is based on the observation that T5dSSs share distinctive features of both T5aSSs and T5bSSs. While being expressed as a single polypeptide like T5aSSs, T5dSSs form an OM $\beta$-barrel composed of 16 anti-parallel $\beta$ strands and possess the afore mentioned single POTRA domain similar to T5bSSs. The prototype of this secretion system is the patatin-like protein D from Pseudomonas aeruginosa, first described in 2010 by 
Salacha et al. (129). Type $5 d$-secreted passengers will be discussed in detail later.

The Type 5e Secretion System: Inverse Autotransporters

Type 5e secretion systems have the same conserved modular architecture as classical autotransporters, including a 12-stranded $\beta$ barrel and a monomeric passenger domain. In contrast to the classical autotransporter, autotransporters of the type 5e subgroup have an inverted domain organization, with the translocator domain at the $\mathrm{N}$ terminus and the passenger domain at the C-terminus $(130,131)$. Like classical autotransporters, inverse autotransporters (IATs) possess an $\mathrm{N}$-terminal signal sequence for Sec-dependent IM transit. In many cases, the signal sequence is longer than the average for Sec-secreted proteins of Gram-negative bacteria (132), also in common with classical autotransporters. In contrast to classical autotransporters, IATs lack the conserved N-terminal extension present in T5aSSs and no clear signal sequence conservation can be identified within the IAT family (132). Thus, the exact function of this elongated signal sequence 
is still unclear. After IM transit, the chaperone SurA plays a major role for transit across the periplasm $(130,133)$ while presumably maintaining a translocation-competent conformation needed for passenger export $(130,134)$. As is common for all autotransporters, the translocation domain of IATs is the most conserved region present in IATs. These highly conserved intimin-like domains, named after its discovery in the translocator domain of E. coli intimin, are the defining characteristic for proteins of the IAT family and were successfully used to identify a wide range of representative members of this family (135). The translocator domain forms a 12-stranded $\beta$-barrel in the OM connected to a short $\mathrm{N}$-terminal periplasmic domain. The periplasmic domain of some IATs, such as Intimin, mediates binding of the autotransporter to peptidoglycan, presumably increasing resistance against mechanical and chemical stress encountered during e.g. transit through the gastrointestinal tract in the case of enteropathogenic bacteria (136). In the case of E. coli Intimin, which forms homodimers in the OM (137), the periplasmic domain also mediates intimin dimerization (136). Following the Bam-dependent OM insertion of the 
$\beta$-barrel, the passenger is translocated via a hairpin intermediate (130), as described in detail for classical autotransporters. In the case of Intimin, and presumably other IATs, passenger secretion is driven by the sequential folding of the extracellular Ig-like domains (95). Following OM transit, the IAT passenger domains remain linked to their respective membrane anchor in contrast to the cleavage of a subset of passenger domains present in classical autotransporters (138).

\section{Type 5d-secreted passengers}

\section{Patatin-like proteins (PLPs)}

Patatin is a plant storage glycoprotein which accounts for approximately $40 \%$ of the total soluble protein in mature potato (Solanum tuberosum) tubers while also exhibiting nonspecific lipid acyl hydrolase activity (139) as well as phospholipase A1 (PLA1) and A2 (PLA2) activity, especially towards phosphatidylcholine (140). The lipolytic activity of patatin is involved in the resistance mechanism against plant pathogens (141) as well as in plant signal transduction 
(142), although other functions are possible. These glycoproteins form homodimers in their natural environment with an approximate molecular weight of 40-43 kDa for the individual subunit (143). Based on sequence homology of conserved domains between potato patatin and the human cytosolic phospholipase A2 (cPLA2) (144), and later confirmed by crystal structure analysis, patatin contains an active-site consisting of a Ser-Asp catalytic dyad, resembling the active site of cPLA2, instead of the Serine-Histidine-Aspartic acid (Ser-His-Asp) catalytic triad most common in lipolytic enzymes (145).

The first patatin-like protein (PLP) identified in bacteria, due to defined regions of protein homology to patatin, was ExoU from Pseudomonas aeruginosa. ExoU belongs to a group of effector proteins, directly translocated into the host cell by a bacterial T3SS. Like patatin, ExoU possesses both lipase as well as PLA activity facilitated by a Ser-Asp catalytic dyad $(146,147)$. Site specific mutagenesis of a single residue in the catalytic dyad, ExoUS142A or ExoUD344A, are enough to render ExoU catalytically inactive resulting in a loss of toxicity (146). After the initial classification of ExoU as PLP, more potential PLPs were detected 
in a wide range of Gram-negative as well as Gram-positive bacteria, with a significantly higher number of PLP genes in genomes of pathogenic bacteria compared to those of non-pathogens (148). Bacterial PLPs possess four conserved domains (blocks I-IV), block I, block II and block IV also found in potato patatin as well as an additional motif, block III (148) (Fig.3). Characteristic for block I is a glycine-rich region containing a conserved arginine or lysin residue serving presumably as an oxyanion hole. Block II harbors a typical sequence motif of $\alpha / \beta$-hydrolases with the putative active-site serine flanked by two glycines (Gly-X-Ser-X-Gly, where $\mathrm{X}$ is any amino acid) (149). Block III contains a conserved serine which is assumed to function as structural element, either as a potential phosphorylation site or due to its ability for hydrogen bond formation. The active-site aspartate residue is located in block IV, which forms the catalytic dyad together with the active-site serine residue located in block II. Block III and block IV in addition consist of highly conserved prolines which are assumed to be important for the proper conformation of the protein (148). Based on their amino acid sequence, and consequently the lack 
of any homology to known groups of bacterial lipases (149), PLPs were classified as a separate group of bacterial lipolytic enzymes (148).

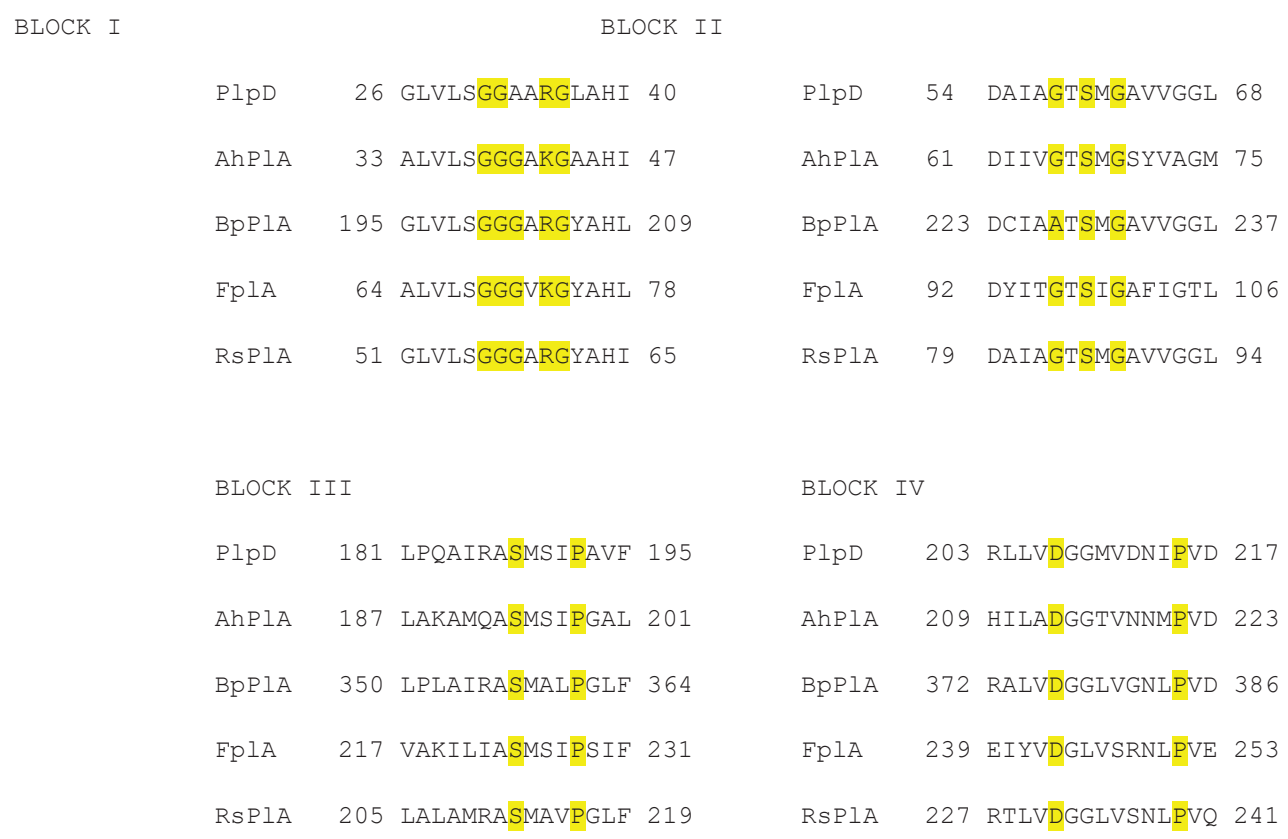

Figure 3: Patatin-like Proteins (PLP) - conserved domains. PLP possess four conserved domains, block I to block IV. Highlighted in yellow are the sequence motifs which are characteristic for each individual block. In the case of Block I, this is a glycine-rich region containing a conserved arginine or lysin residue. Block II shows a conserved active-site serine flanked by two glycines. Block III contains of a conserved serine as well as a conserved proline. Block IV consists of the active-site aspartate in addition to a conserved proline. Phospholipase autotransporters (PIAs) investigated in this thesis were chosen as representative PLPs. PIpD - PIA from Pseudomonas aeruginosa; AhPIA - PIA from Aeromonas hydrophila; BpPIA - PIA from Burkholderia pseudomallei; FpIA - PIA from Fusobacterium nucleatum; RsPIA - PIA from Ralstonia solanacearum. 


\section{Patatin-like Protein D (PIpD)}

Belonging to the group of PLPs, patatin-like protein $D(P \mid p D)$ from Pseudomonas aeruginosa is the prototype of T5dSS (129). The 728residue-long PIpD possesses an $\mathrm{N}$-terminal signal sequence for Secdependent transit across the IM, followed by a passenger domain characteristic for PLPs and a TpsB-like region comprising a single POTRA domain as well as the translocator domain. The passenger domain ( $\left.\mathrm{PlpD}_{20-333}\right)$ displays a typical $\alpha / \beta$-hydrolase fold with eight major helices surrounding a central six-stranded $\beta$-sheet (150) (Fig.4) which is presumably cleaved off and released into the extracellular space upon OM translocation. The active site serine (S60) of $\mathrm{PlpD}_{20-333}$ is located at a highly hydrophobic channel-like region between $\beta 2$ and $\alpha 2$. The active site aspartate (D207) is located at the distal end of a $\beta$ hairpin with its side chain $4.1 \AA$ away from the $\mathrm{OH}$ moiety of Ser60, thus completing the typical lipase active site. The oxyanion hole needed for charge stabilization developed during catalysis consist presumably of two consecutive glycine residue (Gly31, Gly 32) located in close proximity to Ser60 in the loop between $\alpha 1$ and $\beta 1$ (150). Helix 
$\alpha 7$ of $\mathrm{PlpD}_{20-333}$ furthermore directly interacts with its counterpart in a second PIpD molecule facilitating the formation of homodimers as demonstrated by size exclusion chromatography (SEC), analytical centrifugation and protein crystallography (150) (Fig.4).

Enzymatic tests showed clear lipolytic activity for $\mathrm{PlpD}_{20-333}$ toward the non-native substrate $p$-nitrophenol palmitate. Mutagenesis of the active site residue Ser60 to alanine disrupted the catalytic dyad rendering the protein inactive $(150)$. $\mathrm{PlpD}_{20-333}$ also demonstrated PLA1 activity while lacking PLA2 activity, showing the specific hydrolysis of the $s n-1$ position of phospholipids and its capacity to generate fatty acids and lysophospholipids from target membranes (150). An elevated enzymatic activity at $37^{\circ} \mathrm{C}$ and $42^{\circ} \mathrm{C}$ compared to room temperature as well as the interaction of $\mathrm{PlpD}_{20-333}$ with lipids involved in eukaryotic cell signaling, PI, PA and PS, suggested a potential role in the infection process of eukaryotic cells (150). 

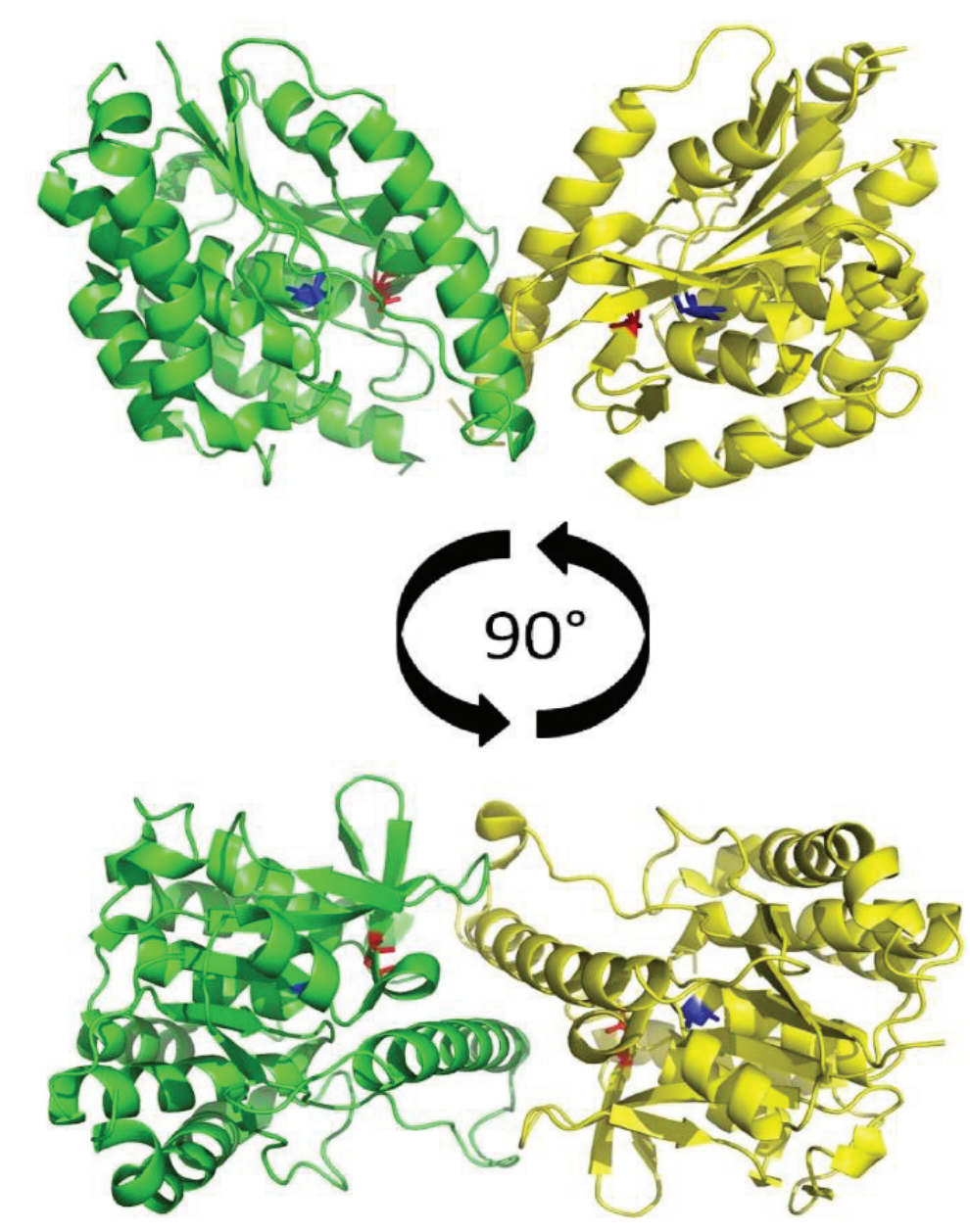

Figure 4. PIpD homodimer. The monomeric passenger domains ( $\mathrm{PlpD}_{20-333}$ ) (colored green and yellow) display a typical $\alpha / \beta$-hydrolase fold with eight major helices surrounding a central six-stranded $\beta$-sheet. Helix $\alpha 7$ of $\mathrm{PlpD}_{20-333}$ furthermore directly interacts with its counterpart in a second PIpD molecule facilitating the formation of homodimers. The active site serine (S60) and aspartate (D207) of each individual protein are indicated in blue and red. PDB: 5fya - Mata Madeira et al., J Mol Biol, 428(9 Pt A):1790-803. (150) 
Fusobacterium phospholipase autotransporter (FplA)

Next to PlpD from Pseudomonas aeruginosa, the Fusobacterium phospholipase autotransporter (FpIA) from Fusobacterium nucleatum was the only other T5dSS PLP to have been characterized in depth (151). FplA is 760 residues in length, with an $\mathrm{N}$-terminal signal sequence required for recognition by the Sec machinery and consequently IM transit, a 40-residue-long $\mathrm{N}$-terminal extension, the patatin-like passenger domain responsible for phospholipase activity, a TpsB-like region comprising a single POTRA domain common in protein-protein interactions and a C-terminal translocator domain. The $\mathrm{N}$-terminal extension described for FplA is absent in PIpD and was the first of its kind to be described for a T5dSS. It was connected to the enzyme's catalytic efficiency and hypothesized to play a critical role in proper protein folding and consequently positioning of the active site residues rather than substrate binding, due to the observation that removal of the $\mathrm{N}$-terminal extension resulted in lower substrate turnover rates $\left(k_{\text {cat }}\right)$ of the artificial substrate 4-methylumbelliferyl heptanoate $(4-\mathrm{MuH})$ while maintaining a Michaelis constant $\left(K_{\mathrm{m}}\right)$ 
comparable to the protein before removal of the $\mathrm{N}$-terminal extension (151). In accordance with T5dSS in general, the translocator domain forms a 16 stranded $\beta$-barrel incorporated into the OM allowing the translocation of the $\mathrm{N}$-terminal passenger domain through the so formed channel across the OM. The passenger domain possesses a catalytic Ser-Asp dyad in addition to an oxyanion hole similar to PlpD. Heterologous FplA stays attached to the OM upon translocation in $E$. coli whereas no FplA could be detected on the surface of $F$. nucleatum. The inability to detect FplA on the surface of its natural host might be a sign for the release of the enzyme into the extracellular space but could also be due to the low abundance of this protein on the cell surface and the limitation of the detection methods used so far. Expression of the full-length protein in different strains of $F$. nucleatum however revealed that the $\mathrm{N}$-terminal passenger domain stays attached to the cell surface in spite of being cleaved upon OM translocation by a yet unknown protease and mechanism. In any case, FpIA possesses lipolytic activity towards the artificial substrate 4-MuH as well as PLA1 activity towards the PLA1-specific substrate PED-A1 
with the highest efficiency at a $\mathrm{pH}$ of 8.5. Specific binding towards phosphatidylinositol phosphates (PIPs), especially $\mathrm{PI}(3,5) \mathrm{P}_{2}$ and $\mathrm{PI}(3,4,5) \mathrm{P}_{3}$, was observed (151), although cleavage of these lipids, which play important roles in cell signaling processes, are yet to be shown.

\section{Glycerophospholipids}

Glycerophospholipids are the most abundant phospholipids and the main structural lipids in membrane bilayers. Glycerophospholipids consist of a hydrophilic head domain and a hydrophobic tail domain. The hydrophobic domain is formed by two fatty acid chains bound to a glycerol moiety via ester bonds, resulting in the diacylglycerol (DAG) backbone of glycerophospholipids. Attached to the DAG backbone is a phosphate group, giving rise to phosphatidic acid (PA), and variable head group, most frequent a choline (resulting in phosphatidyl choline; $\mathrm{PC})$, ethanolamine (PE), serine (PS) or inositol (PI). The length and saturation of the fatty acid chains can vary between different lipids (152). The distribution of these phospholipids across biological 
membranes is asymmetric in eukaryotic $(153,154)$ as well as in prokaryotic cells (155). PC accounts for $\geq 50 \%$ of the phospholipids present in most eukaryotic membranes (152), mostly located within the outer monolayer of the plasma membrane (PM) (156), whereas the inner monolayer consists of PE and the negatively charged PS and PI $(157,158)$. In prokaryotic cells, PE is enriched in the inner membrane, whereas PG is predominantly localized in the outer membrane $(158,159)$. PS is less abundant or absent, but nonetheless an important cytosolic precursor in the synthesis of essential membrane lipids like $\mathrm{PE}$ in $E$. coli via the two enzymes phosphatidylserine synthase and phosphatidylserine decarboxylase (155). Next to its role in PE synthesis, PS is also a known target lipid for a range of specific membrane binding proteins (160). Pls are most abundant in cytosolic membranes and play essential roles in eukaryotes. These roles include their function as membrane-located interaction partners for a wide range of proteins involved in cellular signal transduction cascades $(161,162)$. 
Next to their structural function, lipids can act as secondary messengers in signal transduction pathways (152). Many G-proteincoupled receptors as well as tyrosine kinase-linked receptors activate the PI signaling pathway. In response to an extracellular signal, phospholipase $\mathrm{C}$ is activated which in turn hydrolyses phosphatidylinositol 4,5-biphosphate $\left(\mathrm{PI}, 5 \mathrm{P}_{2}\right)$, located in the $\mathrm{PM}$, into the secondary messengers inositol 1,4,5-triphosphate $\left(\mathrm{IP}_{3}\right)$ and DAG. $I P_{3}$ is connected to an increase in intracellular calcium levels whereas DAG has a positive feedback effect through activation of PLC (163). An increase in intracellular DAG can also be mediated by phospholipase D (PLD)-dependent hydrolysis of PC (164). Next to activation of PLC, many more in vitro effects have been reported connected to DAG as secondary messenger, including the activation of all isozymes of the Protein Kinase C (PKC) family and other proteins with cysteine-rich regions homologous to PKC. The conversion of DAG to PA by the DAG kinase gives rise to yet another lipid considered to be an intracellular signal, although its physiological targets have not been rigorously defined (164). 


\section{In-vivo infection model: Galleria mellonella}

The greater wax-moth, Galleria mellonella, is an insect belonging to the order Lepidoptera. While being a ubiquitous pest of the honeybee (165), it has emerged as an infection model to study virulence factors and pathogenesis of many prominent bacterial and fungal pathogens. As an invertebrate model, larvae of G. mellonella are not subjected to the same ethical constraints as vertebrates, are cheap, easy to handle and allowing for a high throughput of experiments compared to mammalian systems due to the organism `s fast reproduction time. The larvae are dull white in color, roughly $3 \mathrm{~cm}$ in length, weigh approximately 0.3-0.5 g and are relatively large in size (12-20 mm), allowing for intrahemocoelic injection of test compounds. However, a major advantage is the larvae's ability to survive at $37^{\circ} \mathrm{C}$ particularly important when studying mammalian pathogens. So far, there have been several studies confirming a correlation between microbial pathogenicity and virulence determinants in mammals and larvae of G. mellonella (166-168). When it comes to the larvae's immune system, a high degree of structural and functional homology can be 
found when compared to the innate immune system of vertebrates, with both cellular and humoral defense mechanisms (169-171). Thus, larvae of $G$. mellonella have been used to assess the virulence of $P$. aeruginosa (167), Bacillus thuringiensis, B. cereus (168) and Candida albicans (172). These studies demonstrate that studying the insect's response to infection might provide a valid alternative to vertebrate testing while providing comparable data. Despite its advantages, the Galleria model is not as well established as other invertebrate models. Especially the lack of suppliers who offer specific genotypes that were raised under standard conditions, may influence their susceptibility to infections due to differences in genotypes, breeding conditions and maintenance of the larvae (173). Also, the lack of an adaptive immune response limits the applications of this model. 


\section{Aims of the thesis}

Bacterial secretion systems as well as phospholipases are important virulence factors in a variety of bacteria. Compared to many of the other secretion systems, however, knowledge about the T5SSs is still patchy. Especially the T5dSS subgroup of autotransporters, with its archetype PlpD from Pseudomonas aeruginosa, has not been investigated in detail. Here, we aimed to direct the scientific focus on this, so far neglected, secretion system. Orthologs of $p / p D$ are widely distributed among hundreds of other species, both pathogenic as well as environmental. The two T5dSS described to date, PIpD as well as FpIA from Fusobacterium nucleatum, belong to the group of phospholipases often associated with bacterial disease. Thus, the main aim of this project was the characterization and comparison of potential T5dSSs in a range of pathogenic bacteria. A secondary goal was the characterization of the mechanism of type $5 d$ secretion and the evaluation of the role of the type $5 d$-secreted proteins in the pathogenicity of the respective strain. 


\section{Summary of papers}

\section{Paper 1}

Klein K, Sonnabend MS, Frank L, Leibiger K, Franz-Wachtel M, Macek

B, Trunk T et al. Deprivation of the Periplasmic Chaperone SurA

Reduces Virulence and Restores Antibiotic Susceptibility of

Multidrug-Resistant Pseudomonas aeruginosa. Front Microbiol. 2019;10:100. (174)

Pseudomonas aeruginosa is one of the main causative agents of nosocomial infections and the spread of multidrug-resistant strains is rising. Therefore, novel strategies for therapy are urgently required. The outer membrane composition of Gram-negative pathogens and especially of $P$. aeruginosa restricts the efficacy of antibiotic entry into the cell and determines virulence. For efficient outer membrane protein biogenesis, the $\beta$-barrel assembly machinery (Bam) complex in the outer membrane and periplasmic chaperones like Skp and SurA are crucial. Previous studies indicated that the importance of individual proteins involved in outer membrane protein biogenesis may vary between different Gram-negative-negative species. In addition, since 
multidrug-resistant $P$. aeruginosa strains pose a serious global threat, the interference with both virulence and antibiotic resistance by disturbing outer membrane protein biogenesis might be a new strategy to cope with this challenge. Therefore, deletion mutants of the genes coding for non-essential Bam complex components bamB and bamC, of the $s k p$ homolog $h l p A$ as well as a conditional mutant of surA were investigated. The most profound effects for both traits were associated with reduced levels of SurA, characterized by increased membrane permeability, enhanced sensitivity to antibiotic treatment and attenuation of virulence in a Galleria mellonella infection model. Strikingly, the depletion of SurA in a multidrug-resistant clinical bloodstream isolate re-sensitized the strain to antibiotic treatment. From our data we conclude that SurA of $P$. aeruginosa serves as a promising target for developing a drug that shows anti-infective activity and re-sensitizes multidrug-resistant strains to antibiotics. 


\section{Paper 2}

Trunk T, Casasanta MA, Yoo CC, Slade DJ, Leo JC. Comparison of type 5d autotransporter phospholipases demonstrates a correlation between high activity and intracellular pathogenic lifestyle. Biochem J. 2019;476(18):2657-76. (175)

Autotransporters, or type 5 secretion systems, are widespread surface proteins of Gram-negative bacteria often associated with virulence functions. Autotransporters consist of an outer membrane $\beta$-barrel domain and an exported passenger. In the poorly studied type $5 d$ subclass, the passenger is a patatin-like lipase. The prototype of this secretion pathway is PIpD of Pseudomonas aeruginosa, an opportunistic human pathogen. The PIpD passenger is a homodimer with phospholipase A1 (PLA1) activity. Based on sequencing data, PlpD-like proteins are present in many bacterial species. We characterized the enzymatic activity, specific lipid binding and oligomeric status of PIpD homologs from Aeromonas hydrophila (a fish pathogen), Burkholderia pseudomallei (a human pathogen) and Ralstonia solanacearum (a plant pathogen) and compared these with 
PlpD. We demonstrate that recombinant type $5 d$-secreted patatin domains have lipase activity and form dimers or higher-order oligomers. However, dimerization is not necessary for lipase activity; in fact, by making monomeric variants of PlpD, we show that enzymatic activity slightly increases while protein stability decreases. The lipases from the intracellular pathogens $A$. hydrophila and $B$. pseudomallei display PLA2 activity in addition to PLA1 activity. Although the type $5 \mathrm{~d}$-secreted lipases from the animal pathogens bound to intracellular lipid targets, phosphatidylserine and phosphatidylinositol phosphates, hydrolysis of these lipids could only be observed for FplA of Fusobacterium nucleatum. Yet, we noted a correlation between high lipase activity in type $5 \mathrm{~d}$ autotransporters and intracellular lifestyle. We hypothesize that type $5 \mathrm{~d}$ phospholipases are intracellularly active and function in modulation of host cell signaling events. 


\section{Paper 3}

Manuscript

Trunk T, Morth JP, Leo JC. Low resolution structure of the phospholipase autotransporter of Aeromonas hydrophila.

The phospholipase autotransporter from Aeromonas hydrophila (AhPIA) belongs to the group of acylhydrolases and is one of the few members of the subgroup of bacterial type $\mathrm{Vd}$ secretion systems characterized so far. With bacterial phospholipases and type $\mathrm{V}$ secretion systems being important virulence factors in a wide range of extracellular, vacuolar and intracellular pathogens, AhPIA is a good candidate for further investigation. Although sharing high sequence and predicted secondary structure similarities with the lipolytic active patatin-like protein (PlpD) from Pseudomonas aeruginosa, the threedimensional structure of AhPIA is not available to date. In this study, the recombinant catalytic domain of AhPIA was expressed in Escherichia coli and purified in a soluble form. Using the hanging-drop vapor diffusion method, crystals of the purified protein were obtained.

Diffraction data were collected at $6 \AA$ resolution using synchrotron 
radiation. Despite the low resolution is was possible to determine the space group P212121 and possible to solve an initial low-resolution structure of the catalytic domain of AhPIA. This is only the second structure of a type Vd-secreted autotransporter lipase, which confirms the prediction that all type $\mathrm{Vd}$ autotransporter passengers share a patatin-like hydrolase fold. 


\section{Unpublished data}

PlpD is non-essential for pathogenicity of P. aeruginosa in Galleria mellonella

The larvae of the greater wax moth, Galleria mellonella, were employed as in-vivo model to study the role of PlpD during the pathogenicity of $P$. aeruginosa PAO1 and PA14. Homologous recombination was used for the construction of $p / p D$ deletion mutants in both strains (174). Mutant strains as well as the wild type strains were grown in Lysogeny broth (LB) to an $\mathrm{OD}_{600}$ value of 1 followed by serial dilution in PBS to a final concentration of $1 \times 10^{3}$ bacteria $/ \mathrm{mL}$. Galleria mellonella TruLarv $^{\circledR}$ larvae were obtained from BioSystems Technology. Groups of 10 larvae were tested by intrahemocoelic injection (176) of $10 \mu \mathrm{L}$ of the respective strain using a single syringe infusion pump (Cole-Parmer). As negative control, one group was injected with $10 \mu \mathrm{L}$ PBS. Larvae, each weighing 0.2-0.3 g, were kept at $37^{\circ} \mathrm{C}$ for 5 days and were checked for stages of disease/survival at 24 $\mathrm{h}$ intervals. Dead larvae, detectable by strong melanization and lack of movement, were removed from the stock of surviving larvae. 
Except the negative control group, all larvae were found dead $24 \mathrm{~h}$ post infection. The mortality rate of larvae was unaffected by deletion of $p / p D$ in either strain. No mortality was observed in the control group during the 5-day experiment. Thus, we conclude that PIpD is nonessential for pathogenicity of P. aeruginosa PAO1 and PA14 in Galleria mellonella. However, we cannot exclude a role for PlpD during virulence. G. mellonella larvae are highly sensitive to infection with $P$. aeruginosa, leading to a quick death within hours after infection with as few as 10 bacteria/larva. This narrow time frame due to the larvae's high sensitivity towards $P$. aeruginosa might hide any eventual effect resulting from $p / p D$ deletion. Thus, although being an increasingly popular infection model for bacterial as well as fungal pathogens, $G$. mellonella might not be the best model to study the role of the T5dSS during virulence in $P$. aeruginosa. 


\section{Discussion}

\section{SurA}

The permeability barrier of the $\mathrm{OM}$ is the first line of defense in Gramnegative bacteria and essential for the ability to resist extracellular threats. Together with the active export of antimicrobial agents by efflux pumps located in the bacterial cell envelope, this is the main reason behind the high intrinsic resistance of $P$. aeruginosa as well as other Gram-negative bacteria against antibiotics. The conditional deletion of the periplasmic chaperone SurA in Pseudomonas resulted in an alteration of the OM composition and subsequently OM integrity. This in turn increased the accessibility as well as sensitivity to bile salts, antibiotics and serum components. While destabilizing the OM integrity by means of SurA deletion affected expression and incorporation of many porins in a negative fashion, like members of the OprD family of nutritional transporters, it also led to an increase in certain OM porins, like OprM. OprM is associated with the MexAB and MexXY efflux pumps and involved in mediating resistance against $\beta$ lactams, chloramphenicol, macrolides, quinolones, tetracycline and 
aminoglycosides $(177,178)$. The increased expression of certain OM porins in the conditional SurA deletion mutant may be the result of an increased influx of extracellular substances due to a decrease in OM integrity. However, it clearly shows that not all OMPs are dependent on SurA for periplasmic transit.

Next to OM porins, the absence of SurA negatively affected the abundance of siderophore receptors in the OM, which correlates with the findings that SurA depletion strongly affects growth in LB medium under iron-restricted conditions. Another protein effected by SurA depletion in Pseudomonas is the autotransporter PlpD. Although mRNA expression analysis shows continued expression of PlpD, abundance of the protein in the $\mathrm{OM}$ is reduced in the absence of the periplasmic chaperone. This indicates an essential role of SurA during periplasmic transit and/or subsequent incorporation of PIpD into the OM.

The consequences of SurA-mediated alteration of OMP composition and disruption of OM integrity can be manifold. The strain S. enterica serovar Typhimurium BRD115 for example, lacking a functional SurA, 
was found to be defective in the ability to adhere to and invade eukaryotic cells (179). Thus, the surA gene product plays a critical role in folding key proteins and in the proteolytic protection of key proteins directly involved in host-pathogen interactions during the infection process, e.g. structural proteins or effector proteins of the various secretion systems necessary for successful host invasion.

One of those secretion systems might be the T5dSS, which is found in a broad range of bacteria, including the human pathogens $P$. aeruginosa, B. pseudomallei and F. nucleatum, the phytopathogen $R$. solanacearum, and the fish pathogen A. hydrophila. However, the dependency of the respective T5dSSs on the periplasmic chaperone SurA, except for PIpD from $P$. aeruginosa, is still speculative. Yet, it would not come as a surprise, due to the high similarity in primary and secondary structure as well as functional similarities between PIpD in its homologs in the other bacterial species. 


\section{Phospholipase autotransporters}

PlpD is the prototype of T5dSSs, first described roughly a decade ago (129). Full-length PlpD is composed of a single polypeptide with an Nterminal signal sequence for IM transit, followed by the passenger domain, a single POTRA domain and the C-terminal translocator domain. Being expressed in the bacterial cytoplasm, this multi-domain protein must remain in its unfolded, inactive form while being translocated across the IM as well as during periplasmic transit. Once at the periplasmic site of the $\mathrm{OM}$, the pre-protein's translocator domain is assumed to be incorporated into the $\mathrm{OM}$ to form a pore, while the passenger domain has to remain in a translocation compatible form. After OM pore formation, PIpD is believed to facilitate the translocation of its own passenger domain. Following OM transit, the passenger presumably gets cleave off, either remaining attached to the bacterial cell surface or being released into the extracellular space, forming homodimers.

With the focus on pathogenic bacteria, we confirmed and characterized three new phospholipases in the fish pathogen $A$. 
hydrophila, the phytopathogen $R$. solanacearum and the human pathogen B. pseudomallei. All three displayed clear lipolytic activity towards the artificial substrate 4-MuH as well as the PLA1-specific substrate PED-A1, whereas only AhPIA and BpPIA possess activity towards the PLA2-specific substrate BODIPY ${ }^{\circledR}$ PC-A2. Thus, all three PIAs belong to the group of acyl hydrolases, like PIpD and FpIA, with RsPIA belonging to the group of PLA1 and AhPIA as well as BpPIA to the group of PLB. While PLA1 catalyzes the hydrolyses of fatty acids exclusively at the $s n-1$ position of phospholipids, PLB can hydrolyze both the $s n-1$ and the $s n-2$ position. In both cases, the resulting products of the phospholipase reaction is a free fatty acid and a lysophospholipid (180). The remaining fatty acid still connected to the lysophospholipid is in turn cleaved by lysophospholipases (181). Phospholipases exist in most organisms, pathogenic as well as commensal, and their function can be manifold. They may play a role in membrane maintenance and remodeling, act as digestive agent, or regulate cellular mechanisms by creation of bioactive lipid molecules used in signal transduction (180). In the case of PIAs, the 
phospholipase domain is translocated across the OM which makes it unlikely that they are involved in membrane maintenance and remodeling. Therefore, the other two points are more likely, supported by the fact that phospholipases are also known virulence factors involved in phagosomal escape or phagosomal maturation avoidance, favoring tissue colonization, infection establishment and progression, or immune response evasion (182-184). Despite PIAs being highly similar, their exact function may differ between the respective organisms. Probably depending on their function, phospholipases can exist in a secreted, membrane associated or cytoplasmic form and may or may not depend on additional cofactors for activity. The absence of cofactors could be one explanation why most tested PIAs lacked obvious lipolytic activity when confronted with PS or PI(4)P, even though strong binding could be observed by all proteins. An indication for this is found in the BpPIA-mediated digestion of certain lipids present in the commercially available soy extract lipid mix, whereas no digestion could be observed when BpPIA was confronted with purified, single lipid species. Again, the fact that 
FpIA possesses lipolytic activity towards PS and PE also in the absence of any cofactors shows that, despite high homology between the individual PIAs, their substrate specificity and presumably function may differ. Another possibility, supported by the lack of PIA-mediated digestion of any of the major lipids after incubation with AhPIA, BpPIA, PIpD or RsPIA, is that most PIAs tested may target minor lipid species. Thus, they could still be independent of cofactors and may play a role in e.g. modulating host signaling events.

Another common feature of all PIAs tested is the formation homodimers or higher order multimers following translocation across the OM. Although homodimer formation of PlpD had been observed before (150), the question regarding the purpose of homodimer formation remained open. Here, we could show that enzymatic activity of PlpD does not depend on homodimer formation but rather supports protein stability. Through the targeted substitution of important residues in the interaction interface, either $\mathrm{M} 249 \mathrm{E}$ or M256D in combination with I253A, we were able to express stable, enzymatically active monomers. Interestingly, the prevention of 
homodimer formation resulted in a slight increase in enzymatic activity of the monomers compared to the dimer while a clear correlation between a decrease in protein stability and an increase in temperature could be observed. Nonetheless, we cannot exclude that homodimer formation, or in the case of BpPIA, complexes of more than 2 subunits, may play an additional role in e.g. heightened substrate specificity or in the regulation of enzymatic activity.

Despite PIAs being highly similar in structure and primary sequence, several PIAs have a distinct difference in form of a $\mathrm{N}$-terminal extension when compared to PlpD. N-terminal extensions present in BpPIA, RsPIA and FplA are not uniform, but differ in length significantly, with a relatively short extension in the case of RsPIA with only 13 residues, FplA with a 40-residue extension and a long extension in the case of BpPIA with 155 residues. Despite their differences, deletion of $\mathrm{N}$-terminal extension did not result in a significant alteration of enzymatic activity of any of the respective PIAs compared to the fulllength proteins. It did result, however, in a decrease in thermal stability at increasing temperatures in the case of RsPIA and the 
formation of lower molecular-weight complexes in the case of BpPIA. Thus, we speculate that the $\mathrm{N}$-terminal extensions could have a role in protein folding, structural stabilization or possibly in binding to target molecules or membranes. The particularly long $\mathrm{N}$-terminal extension, which is directly linked to the formation of higher molecular-weight complexes, presumably dimer-dimers, are found in the highly virulent B. pseudomallei and B. mallei, whereas lacking in the less pathogenic Burkholderia species, suggesting a role during virulence.

Regarding the question whether or not the individual PIAs are staying attached to the cell surface after translocation across the OM, or are released into the extracellular space, remains open for discussion. While PlpD was reported to be cleaved off and released (129), FplA is cleaved only in certain strains but remains attached to the cell surface (151). However, following heterologous expression in E. coli, neither of the two PIAs is cleaved off $(129,151)$. This led to the assumption that if the passengers are indeed released by proteolysis, this is facilitated by an external protease. Our results indirectly support the idea of PIAs being cleaved off and released into the extracellular space, 
due to the unlikelihood of PIAs forming homodimers or higher molecular-weight complexes while staying surface-attached.

Interestingly, the activities of the various type $5 d$ lipases towards 4MuH are correlated with the lifestyle of the source bacteria. The observation that PIAs from mainly extracellular pathogens like $P$. aeruginosa had relatively low enzymatic activity compared to PIAs from intracellular pathogens like F. nucleatum (147), B. pseudomallei or the facultative intracellular pathogen A. hydrophila, may indicate a primarily intracellular role for type $5 \mathrm{~d}$ phospholipases. Also, testing of the direct toxic effect of the purified PIAs by means of intrahemocoelic injection into larvae of the greater wax moth, Galleria mellonella, showed only low toxicity of these proteins, demonstrating that PIAs are most likely not membrane-disrupting toxins. Of the type $5 d$ phospholipases, only $A$. hydrophila and B. pseudomallei, both possessing PLB activity, showed any toxic effect, pointing to a more pronounced function during virulence. Even then, toxicity required very high protein concentrations. When we tested the effect of $p / p D$ deletion on the pathogenicity of $P$. aeruginosa PAO1 and PA14 during 
bacterial infection of $G$. mellonella larvae, no difference between the deletion mutant and the wild-type could be observed. Although, any effect of the $p / p D$ deletion could have been hidden by the high sensitivity of this model towards Pseudomonas infection, associated with a high mortality resulting from a minimal bacterial load, we concluded that the T5dSS in $P$. aeruginosa is non-essential for pathogenicity in Galleria mellonella.

Taken together, the higher activity of PIAs from intracellular pathogens, the apparently narrow substrate range and the low toxicity of PIAs point towards a very specific, possibly intracellular role for these proteins in virulence.

\section{AhPIA}

While all PIAs investigated in this thesis share a high sequence and predicted secondary structure similarity, distinct differences between the individual proteins can be observed and the sole structure of a PIA solved outside of this thesis was PIpD from P. aeruginosa. Especially 
the differences in enzymatic activity towards PLA-2 specific substrates in AhPIA and BpPIA compared to their close homologs PIpD, FplA and RsPIA, as well as differences in potential interaction partners and the substrate range of the individual enzymes, remained enigmatic. To address yet open questions regarding those differences, protein crystallography was employed to solve the structure of AhPIA. Diffraction-quality crystals of the purified protein were obtained and diffraction data were collected to $6 \AA$ resolution. This low-resolution structure was sufficient to confirm that AhPIA shares the same patatinlike hydrolase fold as PIpD but was insufficient to distinguish specific structural elements responsible for PLA-2 activity or differences in substrate specificity. 


\section{Future directions}

- To investigate the role of the single POTRA domain present in T5dSS and test the hypothesis that T5dSS are an evolutionary intermediate between T5aSS and T5bSS. Other hypothesis, based on the targeting role of the POTRA motif in the TpsB transporter, consider a role as chaperone for the passenger, in the recruitment of proteases for passenger cleavage or in passenger secretion (129). This could be achieved by deletion or substitution of the POTRA domain with e.g. TPS system or BamA POTRA domains.

- To investigate the exact mechanism of passenger secretion and identify all periplasmic and OM located factors involved. The question if the passengers of T5dSS are translocated across the OM via hairpin formation as in T5aSS and T5eSS could be addressed by catching a secretion intermediate and test for extracellular protease accessibility or by use of the SpyTagtechnology hatlem (185). 
- To crystallize and determine high-resolution structures for more PIAs, identifying details responsible for the differences observed in substrate specificity, activity and multimer formation between the individual PIAs despite the high sequence similarity shared by all PIAs.

- To identify the true substrates of the individual PIAs and uncover their role for bacterial fitness, competition or virulence.

- To investigate the potential effect of PIpD on virulence in a vertebrate model, e.g. zebrafish embryos, or by means of leaf infiltration assays in Arabidopsis thaliana, to fully elucidate the function(s) of this class of autotransported enzymes. 


\section{References}

1. Todar's Online Textbook of Bacteriology [Internet]. http://textbookofbacteriology.net/pseudomonas.html. 2007. 2. Lamas Ferreiro JL, Alvarez Otero J, Gonzalez Gonzalez L, Novoa Lamazares L, Arca Blanco A, Bermudez Sanjurjo JR, et al. Pseudomonas aeruginosa urinary tract infections in hospitalized patients: Mortality and prognostic factors. PLoS One. 2017;12(5):e0178178.

3. Fujitani S, Sun HY, Yu VL, Weingarten JA. Pneumonia due to Pseudomonas aeruginosa: part I: epidemiology, clinical diagnosis, and source. Chest. 2011;139(4):909-19.

4. Gellatly SL, Hancock RE. Pseudomonas aeruginosa: new insights into pathogenesis and host defenses. Pathog Dis. 2013;67(3):159-73.

5. Lin TI, Huang YF, Liu PY, Chou CA, Chen YS, Chen YY, et al. Pseudomonas aeruginosa infective endocarditis in patients who do not use intravenous drugs: Analysis of risk factors and treatment outcomes. J Microbiol Immunol Infect. 2016;49(4):516-22.

6. Jacobs RF, McCarthy RE, Elser JM. Pseudomonas osteochondritis complicating puncture wounds of the foot in children: a 10-year evaluation. J Infect Dis. 1989;160(4):657-61.

7. Matsumoto K. Role of bacterial proteases in pseudomonal and serratial keratitis. Biol Chem. 2004;385(11):1007-16.

8. Azghani AO, Kondepudi AY, Johnson AR. Interaction of Pseudomonas aeruginosa with human lung fibroblasts: role of bacterial elastase. Am J Respir Cell Mol Biol. 1992;6(6):652-7.

9. Bardoel BW, van der Ent S, Pel MJ, Tommassen J, Pieterse CM, van Kessel KP, et al. Pseudomonas evades immune recognition of flagellin in both mammals and plants. PLoS Pathog. 2011;7(8):e1002206.

10. Sadikot RT, Blackwell TS, Christman JW, Prince AS. Pathogen-host interactions in Pseudomonas aeruginosa pneumonia. Am J Respir Crit Care Med. 2005;171(11):1209-23.

11. Wiersinga WJ, van der Poll T, White NJ, Day NP, Peacock SJ.

Melioidosis: insights into the pathogenicity of Burkholderia pseudomallei. Nat Rev Microbiol. 2006;4(4):272-82.

12. Schweizer HP. Mechanisms of antibiotic resistance in Burkholderia pseudomallei: implications for treatment of melioidosis. Future Microbiol. 2012;7(12):1389-99.

13. Rhodes KA, Schweizer HP. Antibiotic resistance in Burkholderia species. Drug Resist Updat. 2016;28:82-90. 
14. Stevens MP, Wood MW, Taylor LA, Monaghan P, Hawes $\mathrm{P}$, Jones PW, et al. An Inv/Mxi-Spa-like type III protein secretion system in Burkholderia pseudomallei modulates intracellular behaviour of the pathogen. Mol Microbiol. 2002;46(3):649-59.

15. Vander Broek CW, Stevens JM. Type III Secretion in the Melioidosis Pathogen Burkholderia pseudomallei. Front Cell Infect Microbiol. 2017;7:255.

16. Galan JE, Lara-Tejero M, Marlovits TC, Wagner S. Bacterial type III secretion systems: specialized nanomachines for protein delivery into target cells. Annu Rev Microbiol. 2014;68:415-38.

17. Stevens MP, Haque A, Atkins T, Hill J, Wood MW, Easton A, et al. Attenuated virulence and protective efficacy of a Burkholderia pseudomallei bsa type III secretion mutant in murine models of melioidosis. Microbiology. 2004;150(Pt 8):2669-76.

18. Reckseidler SL, DeShazer D, Sokol PA, Woods DE. Detection of bacterial virulence genes by subtractive hybridization: identification of capsular polysaccharide of Burkholderia pseudomallei as a major virulence determinant. Infect Immun. 2001;69(1):34-44.

19. Reckseidler-Zenteno SL, DeVinney R, Woods DE. The capsular polysaccharide of Burkholderia pseudomallei contributes to survival in serum by reducing complement factor C3b deposition. Infect Immun. 2005;73(2):1106-15.

20. Ashdown LR, Koehler JM. Production of hemolysin and other extracellular enzymes by clinical isolates of Pseudomonas pseudomallei. J Clin Microbiol. 1990;28(10):2331-4.

21. Hayward AC. The Hosts of Pseudomonas solanacearum. In: Hayward ACaH, G.L., editor. Bacterial Wilt: The Disease and Its Causative Agent, Pseudomonas solanacearum. CAB International, Wallingford1994. p. 9-24.

22. Vasse J, Frey, P., Trigalet, A. Microscopic studies of intercellular infection and protoxylem invasion of tomato roots by Pseudomonas solanacearum. Mol Plant Microbe Interact. 1995(8):241-51.

23. Peeters N, Guidot A, Vailleau F, Valls M. Ralstonia solanacearum, a widespread bacterial plant pathogen in the post-genomic era. Mol Plant Pathol. 2013;14(7):651-62.

24. Coll NS, Valls M. Current knowledge on the Ralstonia solanacearum type III secretion system. Microb Biotechnol. 2013;6(6):614-20.

25. Araud-Razou I, Vasse, J., Montrozier, H., Etchebar, C., Trigalet, A. Detection and Visualization of the Major Acidic Exopolysaccharide of 
Ralstonia solanacearum and its Role in Tomato Root Infection and Vascular Colonization. European Journal of Plant Pathology. 1998;104(8):795-809.

26. Genin S, Denny TP. Pathogenomics of the Ralstonia solanacearum species complex. Annu Rev Phytopathol. 2012;50:67-89.

27. Janda JM, Abbott SL. The genus Aeromonas: taxonomy, pathogenicity, and infection. Clin Microbiol Rev. 2010;23(1):35-73.

28. Glunder G, Siegmann O. Occurrence of Aeromonas hydrophila in wild birds. Avian Pathol. 1989;18(4):685-95.

29. Praveen PK, Debnath C, Shekhar S, Dalai N, Ganguly S. Incidence of Aeromonas spp. infection in fish and chicken meat and its related public health hazards: A review. Vet World. 2016;9(1):6-11.

30. Igbinosa IH, Igumbor EU, Aghdasi F, Tom M, Okoh Al. Emerging Aeromonas species infections and their significance in public health. ScientificWorldJournal. 2012;2012:625023.

31. Tan E, Low KW, Wong WS, Leung KY. Internalization of Aeromonas hydrophila by fish epithelial cells can be inhibited with a tyrosine kinase inhibitor. Microbiology. 1998;144 ( Pt 2):299-307.

32. Tomas JM. The main Aeromonas pathogenic factors. ISRN Microbiol. 2012;2012:256261.

33. Rasmussen-Ivey CR, Figueras MJ, McGarey D, Liles MR. Virulence Factors of Aeromonas hydrophila: In the Wake of Reclassification. Front Microbiol. 2016;7:1337.

34. dos Santos PA, Pereira AC, Ferreira AF, de Mattos Alves MA, Rosa $A C$, Freitas-Almeida AC. Adhesion, invasion, intracellular survival and cytotoxic activity of strains of Aeromonas spp. in HEp-2, Caco-2 and T-84 cell lines. Antonie Van Leeuwenhoek. 2015;107(5):1225-36.

35. Beaz-Hidalgo R, Figueras MJ. Aeromonas spp. whole genomes and virulence factors implicated in fish disease. J Fish Dis. 2013;36(4):371-88.

36. Cahill MM. Virulence factors in motile Aeromonas species. J Appl Bacteriol. 1990;69(1):1-16.

37. Tanoue N, Takahashi A, Okamoto K, Fujii Y, Taketani Y, Harada N, et al. A pore-forming toxin produced by Aeromonas sobria activates CAMPdependent $\mathrm{Cl}$ - secretory pathways to cause diarrhea. FEMS Microbiol Lett. 2005;242(2):195-201.

38. Chopra AK, Xu X, Ribardo D, Gonzalez M, Kuhl K, Peterson JW, et al. The cytotoxic enterotoxin of Aeromonas hydrophila induces proinflammatory cytokine production and activates arachidonic acid metabolism in macrophages. Infect Immun. 2000;68(5):2808-18. 
39. Li J, Ni XD, Liu YJ, Lu CP. Detection of three virulence genes alt, ahp and aerA in Aeromonas hydrophila and their relationship with actual virulence to zebrafish. J Appl Microbiol. 2011;110(3):823-30.

40. Sha J, Kozlova EV, Chopra AK. Role of various enterotoxins in Aeromonas hydrophila-induced gastroenteritis: generation of enterotoxin gene-deficient mutants and evaluation of their enterotoxic activity. Infect Immun. 2002;70(4):1924-35.

41. Anguita J, Rodriguez Aparicio LB, Naharro G. Purification, gene cloning, amino acid sequence analysis, and expression of an extracellular lipase from an Aeromonas hydrophila human isolate. Appl Environ Microbiol. 1993;59(8):2411-7.

42. Merino S, Aguilar A, Nogueras MM, Regue M, Swift S, Tomas JM. Cloning, sequencing, and role in virulence of two phospholipases (A1 and C) from mesophilic Aeromonas sp. serogroup 0:34. Infect Immun. 1999;67(8):4008-13.

43. Yu HB, Rao PS, Lee HC, Vilches S, Merino S, Tomas JM, et al. A type III secretion system is required for Aeromonas hydrophila $\mathrm{AH}-1$ pathogenesis. Infect Immun. 2004;72(3):1248-56.

44. Suarez G, Sierra JC, Sha J, Wang S, Erova TE, Fadl AA, et al. Molecular characterization of a functional type $\mathrm{VI}$ secretion system from a clinical isolate of Aeromonas hydrophila. Microb Pathog. 2008;44(4):34461.

45. Brennan CA, Garrett WS. Fusobacterium nucleatum - symbiont, opportunist and oncobacterium. Nat Rev Microbiol. 2019;17(3):156-66. 46. Binder Gallimidi A, Fischman S, Revach B, Bulvik R, Maliutina A, Rubinstein AM, et al. Periodontal pathogens Porphyromonas gingivalis and Fusobacterium nucleatum promote tumor progression in an oral-specific chemical carcinogenesis model. Oncotarget. 2015;6(26):22613-23.

47. Taxman DJ, Swanson KV, Broglie PM, Wen H, Holley-Guthrie E, Huang MT, et al. Porphyromonas gingivalis mediates inflammasome repression in polymicrobial cultures through a novel mechanism involving reduced endocytosis. J Biol Chem. 2012;287(39):32791-9.

48. Han YW. Fusobacterium nucleatum: a commensal-turned pathogen. Curr Opin Microbiol. 2015;23:141-7.

49. Kaplan A, Kaplan CW, He X, McHardy I, Shi W, Lux R.

Characterization of aid1, a novel gene involved in Fusobacterium nucleatum interspecies interactions. Microb Ecol. 2014;68(2):379-87.

50. Kaplan CW, Lux R, Haake SK, Shi W. The Fusobacterium nucleatum outer membrane protein RadD is an arginine-inhibitable adhesin required 
for inter-species adherence and the structured architecture of multispecies biofilm. Mol Microbiol. 2009;71(1):35-47.

51. Bachrach G, lanculovici C, Naor R, Weiss El. Fluorescence based measurements of Fusobacterium nucleatum coaggregation and of fusobacterial attachment to mammalian cells. FEMS Microbiol Lett. 2005;248(2):235-40.

52. Han YW, Shi W, Huang GT, Kinder Haake S, Park NH, Kuramitsu H, et al. Interactions between periodontal bacteria and human oral epithelial cells: Fusobacterium nucleatum adheres to and invades epithelial cells. Infect Immun. 2000;68(6):3140-6.

53. Xu M, Yamada M, Li M, Liu H, Chen SG, Han YW. FadA from Fusobacterium nucleatum utilizes both secreted and nonsecreted forms for functional oligomerization for attachment and invasion of host cells. J Biol Chem. 2007;282(34):25000-9.

54. Green ER, Mecsas J. Bacterial Secretion Systems: An Overview. Microbiol Spectr. 2016;4(1).

55. Costa TR, Felisberto-Rodrigues C, Meir A, Prevost MS, Redzej A, Trokter M, et al. Secretion systems in Gram-negative bacteria: structural and mechanistic insights. Nat Rev Microbiol. 2015;13(6):343-59.

56. Fan E, Chauhan N, Udatha DB, Leo JC, Linke D. Type V Secretion Systems in Bacteria. Microbiol Spectr. 2016;4(1).

57. Leo JC, Grin I, Linke D. Type V secretion: mechanism(s) of autotransport through the bacterial outer membrane. Philos Trans R Soc Lond B Biol Sci. 2012;367(1592):1088-101.

58. Zilkenat S, Franz-Wachtel M, Stierhof YD, Galan JE, Macek B, Wagner S. Determination of the Stoichiometry of the Complete Bacterial Type III Secretion Needle Complex Using a Combined Quantitative Proteomic Approach. Mol Cell Proteomics. 2016;15(5):1598-609.

59. Natale P, Bruser T, Driessen AJ. Sec- and Tat-mediated protein secretion across the bacterial cytoplasmic membrane--distinct translocases and mechanisms. Biochim Biophys Acta. 2008;1778(9):1735-56.

60. Driessen AJ, Manting EH, van der Does C. The structural basis of protein targeting and translocation in bacteria. Nat Struct Biol. 2001;8(6):492-8.

61. Berks BC, Palmer T, Sargent F. Protein targeting by the bacterial twin-arginine translocation (Tat) pathway. Curr Opin Microbiol. 2005;8(2):174-81.

62. Fekkes $\mathrm{P}$, Driessen AJ. Protein targeting to the bacterial cytoplasmic membrane. Microbiol Mol Biol Rev. 1999;63(1):161-73. 
63. Hanada M, Nishiyama KI, Mizushima S, Tokuda H. Reconstitution of an efficient protein translocation machinery comprising SecA and the three membrane proteins, SecY, SecE, and SecG (p12). J Biol Chem.

1994;269(38):23625-31.

64. Douville K, Price A, Eichler J, Economou A, Wickner W. SecYEG and SecA are the stoichiometric components of preprotein translocase. J Biol Chem. 1995;270(34):20106-11.

65. Brundage L, Hendrick JP, Schiebel E, Driessen AJ, Wickner W. The purified E. coli integral membrane protein SecY/E is sufficient for reconstitution of SecA-dependent precursor protein translocation. Cell. 1990;62(4):649-57.

66. Henderson IR, Navarro-Garcia F, Nataro JP. The great escape: structure and function of the autotransporter proteins. Trends Microbiol. 1998;6(9):370-8.

67. Hartl FU, Lecker S, Schiebel E, Hendrick JP, Wickner W. The binding cascade of SecB to SecA to SecY/E mediates preprotein targeting to the $E$. coli plasma membrane. Cell. 1990;63(2):269-79.

68. Economou A, Wickner W. SecA promotes preprotein translocation by undergoing ATP-driven cycles of membrane insertion and deinsertion. Cell. 1994;78(5):835-43.

69. Schiebel E, Driessen AJ, Hartl FU, Wickner W. Delta mu H+ and ATP function at different steps of the catalytic cycle of preprotein translocase. Cell. 1991;64(5):927-39.

70. Veenendaal AK, van der Does C, Driessen AJ. The proteinconducting channel SecYEG. Biochim Biophys Acta. 2004;1694(1-3):81-95.

71. Dalbey RE, Wang P, Kuhn A. Assembly of bacterial inner membrane proteins. Annu Rev Biochem. 2011;80:161-87.

72. Angelini S, Deitermann S, Koch HG. FtsY, the bacterial signalrecognition particle receptor, interacts functionally and physically with the SecYEG translocon. EMBO Rep. 2005;6(5):476-81.

73. Koebnik R, Locher KP, Van Gelder P. Structure and function of bacterial outer membrane proteins: barrels in a nutshell. Mol Microbiol. 2000;37(2):239-53.

74. Sklar JG, Wu T, Kahne D, Silhavy TJ. Defining the roles of the periplasmic chaperones SurA, Skp, and DegP in Escherichia coli. Genes Dev. 2007;21(19):2473-84.

75. Wu T, Malinverni J, Ruiz N, Kim S, Silhavy TJ, Kahne D. Identification of a multicomponent complex required for outer membrane biogenesis in Escherichia coli. Cell. 2005;121(2):235-45. 
76. Sklar JG, Wu T, Gronenberg LS, Malinverni JC, Kahne D, Silhavy TJ. Lipoprotein SmpA is a component of the YaeT complex that assembles outer membrane proteins in Escherichia coli. Proc Natl Acad Sci U S A. 2007;104(15):6400-5.

77. Voulhoux R, Bos MP, Geurtsen J, Mols M, Tommassen J. Role of a highly conserved bacterial protein in outer membrane protein assembly. Science. 2003;299(5604):262-5.

78. Han L, Zheng J, Wang Y, Yang X, Liu Y, Sun C, et al. Structure of the BAM complex and its implications for biogenesis of outer-membrane proteins. Nat Struct Mol Biol. 2016;23(3):192-6.

79. Gu Y, Li H, Dong H, Zeng Y, Zhang Z, Paterson NG, et al. Structural basis of outer membrane protein insertion by the BAM complex. Nature. 2016;531(7592):64-9.

80. Bakelar J, Buchanan SK, Noinaj N. The structure of the beta-barrel assembly machinery complex. Science. 2016;351(6269):180-6.

81. Noinaj N, Kuszak AJ, Balusek C, Gumbart JC, Buchanan SK. Lateral opening and exit pore formation are required for BamA function. Structure. 2014;22(7):1055-62.

82. Noinaj N, Kuszak AJ, Gumbart JC, Lukacik P, Chang H, Easley NC, et al. Structural insight into the biogenesis of beta-barrel membrane proteins. Nature. 2013;501(7467):385-90.

83. Fleming PJ, Patel DS, Wu EL, Qi Y, Yeom MS, Sousa MC, et al. BamA POTRA Domain Interacts with a Native Lipid Membrane Surface. Biophys J. 2016;110(12):2698-709.

84. Doyle MT, Bernstein HD. Bacterial outer membrane proteins assemble via asymmetric interactions with the BamA beta-barrel. Nat Commun. 2019;10(1):3358.

85. Nikaido H. Molecular basis of bacterial outer membrane permeability revisited. Microbiol Mol Biol Rev. 2003;67(4):593-656.

86. Allen WJ, Phan G, Waksman G. Structural biology of periplasmic chaperones. Adv Protein Chem Struct Biol. 2009;78:51-97.

87. Ellis RJ. Steric chaperones. Trends Biochem Sci. 1998;23(2):43-5.

88. Pauwels K, Lustig A, Wyns L, Tommassen J, Savvides SN, Van Gelder $P$. Structure of a membrane-based steric chaperone in complex with its lipase substrate. Nat Struct Mol Biol. 2006;13(4):374-5.

89. Behrens S, Maier R, de Cock H, Schmid FX, Gross CA. The SurA periplasmic PPlase lacking its parvulin domains functions in vivo and has chaperone activity. EMBO J. 2001;20(1-2):285-94. 
90. Spiess C, Beil A, Ehrmann M. A temperature-dependent switch from chaperone to protease in a widely conserved heat shock protein. Cell. 1999;97(3):339-47.

91. leva R, Bernstein HD. Interaction of an autotransporter passenger domain with BamA during its translocation across the bacterial outer membrane. Proc Natl Acad Sci U S A. 2009;106(45):19120-5.

92. Sauri A, Soprova Z, Wickstrom D, de Gier JW, Van der Schors RC, Smit AB, et al. The Bam (Omp85) complex is involved in secretion of the autotransporter haemoglobin protease. Microbiology. 2009;155(Pt 12):3982-91.

93. leva R, Tian P, Peterson JH, Bernstein HD. Sequential and spatially restricted interactions of assembly factors with an autotransporter beta domain. Proc Natl Acad Sci U S A. 2011;108(31):E383-91.

94. Klauser T, Pohlner J, Meyer TF. The secretion pathway of IgA protease-type proteins in gram-negative bacteria. Bioessays. 1993;15(12):799-805.

95. Leo JC, Oberhettinger P, Yoshimoto S, Udatha DB, Morth JP, Schutz $M$, et al. Secretion of the Intimin Passenger Domain is driven by Protein Folding. J Biol Chem. 2016.

96. Choi PS, Bernstein HD. Sequential translocation of an Escherchia coli two-partner secretion pathway exoprotein across the inner and outer membranes. Mol Microbiol. 2010;75(2):440-51.

97. Junker M, Schuster CC, McDonnell AV, Sorg KA, Finn MC, Berger B, et al. Pertactin beta-helix folding mechanism suggests common themes for the secretion and folding of autotransporter proteins. Proc Natl Acad Sci U S A. 2006;103(13):4918-23.

98. Kang'ethe W, Bernstein HD. Charge-dependent secretion of an intrinsically disordered protein via the autotransporter pathway. Proc Natl Acad Sci U S A. 2013;110(45):E4246-55.

99. Mulks MH, Plaut AG. IgA protease production as a characteristic distinguishing pathogenic from harmless neisseriaceae. N Engl J Med. 1978;299(18):973-6.

100. Pohlner J, Halter R, Beyreuther K, Meyer TF. Gene structure and extracellular secretion of Neisseria gonorrhoeae IgA protease. Nature. 1987;325(6103):458-62.

101. Meuskens I, Saragliadis A, Leo JC, Linke D. Type V Secretion Systems: An Overview of Passenger Domain Functions. Front Microbiol. 2019;10:1163.

102. Henderson IR, Nataro JP. Virulence functions of autotransporter proteins. Infect Immun. 2001;69(3):1231-43. 
103. Sijbrandi R, Urbanus ML, ten Hagen-Jongman CM, Bernstein HD, Oudega B, Otto BR, et al. Signal recognition particle (SRP)-mediated targeting and Sec-dependent translocation of an extracellular Escherichia coli protein. J Biol Chem. 2003;278(7):4654-9.

104. Szabady RL, Peterson JH, Skillman KM, Bernstein HD. An unusual signal peptide facilitates late steps in the biogenesis of a bacterial autotransporter. Proc Natl Acad Sci U S A. 2005;102(1):221-6.

105. Ruiz-Perez F, Henderson IR, Leyton DL, Rossiter AE, Zhang Y, Nataro JP. Roles of periplasmic chaperone proteins in the biogenesis of serine protease autotransporters of Enterobacteriaceae. J Bacteriol. 2009;191(21):6571-83.

106. Ruiz-Perez F, Henderson IR, Nataro JP. Interaction of FkpA, a peptidyl-prolyl cis/trans isomerase with EspP autotransporter protein. Gut Microbes. 2010;1(5):339-44.

107. Neuwald AF, Liu JS, Lawrence CE. Gibbs motif sampling: detection of bacterial outer membrane protein repeats. Protein Sci. 1995;4(8):1618-32. 108. Bernstein HD. Type V Secretion in Gram-Negative Bacteria. EcoSal Plus. 2019;8(2).

109. Junker M, Besingi RN, Clark PL. Vectorial transport and folding of an autotransporter virulence protein during outer membrane secretion. Mol Microbiol. 2009;71(5):1323-32.

110. Peterson JH, Tian P, leva R, Dautin N, Bernstein HD. Secretion of a bacterial virulence factor is driven by the folding of a C-terminal segment. Proc Natl Acad Sci U S A. 2010;107(41):17739-44.

111. Soprova Z, Sauri A, van Ulsen P, Tame JR, den Blaauwen T, Jong WS, et al. A conserved aromatic residue in the autochaperone domain of the autotransporter $\mathrm{Hbp}$ is critical for initiation of outer membrane translocation. J Biol Chem. 2010;285(49):38224-33.

112. Dautin N, Barnard TJ, Anderson DE, Bernstein HD. Cleavage of a bacterial autotransporter by an evolutionarily convergent autocatalytic mechanism. EMBO J. 2007;26(7):1942-52.

113. Jacob-Dubuisson F, Locht C, Antoine R. Two-partner secretion in Gram-negative bacteria: a thrifty, specific pathway for large virulence proteins. Mol Microbiol. 2001;40(2):306-13.

114. Ward CK, Mock JR, Hansen EJ. The LspB protein is involved in the secretion of the LspA1 and LspA2 proteins by Haemophilus ducreyi. Infect Immun. 2004;72(4):1874-84.

115. Julio SM, Cotter PA. Characterization of the filamentous hemagglutinin-like protein FhaS in Bordetella bronchiseptica. Infect Immun. 2005;73(8):4960-71. 
116. McCann JR, St Geme JW, 3rd. The HMW1C-like glycosyltransferases-an enzyme family with a sweet tooth for simple sugars. PLoS Pathog. 2014;10(4):e1003977.

117. Grass S, Buscher AZ, Swords WE, Apicella MA, Barenkamp SJ, Ozchlewski N, et al. The Haemophilus influenzae HMW1 adhesin is glycosylated in a process that requires HMW1C and phosphoglucomutase, an enzyme involved in lipooligosaccharide biosynthesis. Mol Microbiol. 2003;48(3):737-51.

118. St Geme JW, 3rd, Grass S. Secretion of the Haemophilus influenzae HMW1 and HMW2 adhesins involves a periplasmic intermediate and requires the HMWB and HMWC proteins. Mol Microbiol. 1998;27(3):61730.

119. Baud C, Hodak H, Willery E, Drobecq H, Locht C, Jamin M, et al. Role of DegP for two-partner secretion in Bordetella. Mol Microbiol. 2009;74(2):315-29.

120. Delattre AS, Saint N, Clantin B, Willery E, Lippens G, Locht C, et al. Substrate recognition by the POTRA domains of TpsB transporter FhaC. Mol Microbiol. 2011;81(1):99-112.

121. Mazar J, Cotter PA. New insight into the molecular mechanisms of two-partner secretion. Trends Microbiol. 2007;15(11):508-15.

122. Meng G, Surana NK, St Geme JW, 3rd, Waksman G. Structure of the outer membrane translocator domain of the Haemophilus influenzae Hia trimeric autotransporter. EMBO J. 2006;25(11):2297-304.

123. Linke D, Riess T, Autenrieth IB, Lupas A, Kempf VA. Trimeric autotransporter adhesins: variable structure, common function. Trends Microbiol. 2006;14(6):264-70.

124. Szczesny P, Lupas A. Domain annotation of trimeric autotransporter adhesins--daTAA. Bioinformatics. 2008;24(10):1251-6.

125. Hartmann MD, Grin I, Dunin-Horkawicz S, Deiss S, Linke D, Lupas AN, et al. Complete fiber structures of complex trimeric autotransporter adhesins conserved in enterobacteria. Proc Natl Acad Sci U S A. 2012;109(51):20907-12.

126. Grosskinsky U, Schutz M, Fritz M, Schmid Y, Lamparter MC, Szczesny $P$, et al. A conserved glycine residue of trimeric autotransporter domains plays a key role in Yersinia adhesin A autotransport. J Bacteriol. 2007;189(24):9011-9.

127. Lehr U, Schutz M, Oberhettinger P, Ruiz-Perez F, Donald JW, Palmer $\mathrm{T}$, et al. C-terminal amino acid residues of the trimeric autotransporter adhesin YadA of Yersinia enterocolitica are decisive for its recognition and assembly by BamA. Mol Microbiol. 2010;78(4):932-46. 
128. Muhlenkamp M, Oberhettinger P, Leo JC, Linke D, Schutz MS. Yersinia adhesin A (YadA)--beauty \& beast. Int J Med Microbiol. 2015;305(2):252-8.

129. Salacha R, Kovacic F, Brochier-Armanet C, Wilhelm S, Tommassen J, Filloux A, et al. The Pseudomonas aeruginosa patatin-like protein PlpD is the archetype of a novel Type V secretion system. Environ Microbiol. 2010;12(6):1498-512.

130. Oberhettinger P, Leo JC, Linke D, Autenrieth IB, Schutz MS. The inverse autotransporter intimin exports its passenger domain via a hairpin intermediate. J Biol Chem. 2015;290(3):1837-49.

131. Oberhettinger P, Schutz M, Leo JC, Heinz N, Berger J, Autenrieth IB, et al. Intimin and invasin export their $\mathrm{C}$-terminus to the bacterial cell surface using an inverse mechanism compared to classical autotransport. PLoS One. 2012;7(10):e47069.

132. Tsai JC, Yen MR, Castillo R, Leyton DL, Henderson IR, Saier MH, Jr. The bacterial intimins and invasins: a large and novel family of secreted proteins. PLoS One. 2010;5(12):e14403.

133. Bodelon G, Marin E, Fernandez LA. Role of periplasmic chaperones and BamA (YaeT/Omp85) in folding and secretion of intimin from enteropathogenic Escherichia coli strains. J Bacteriol. 2009;191(16):516979.

134. Adams TM, Wentzel A, Kolmar H. Intimin-mediated export of passenger proteins requires maintenance of a translocation-competent conformation. J Bacteriol. 2005;187(2):522-33.

135. Fairman JW, Dautin N, Wojtowicz D, Liu W, Noinaj N, Barnard TJ, et al. Crystal structures of the outer membrane domain of intimin and invasin from enterohemorrhagic $\mathrm{E}$. coli and enteropathogenic $\mathrm{Y}$. pseudotuberculosis. Structure. 2012;20(7):1233-43.

136. Leo JC, Oberhettinger $P$, Chaubey $M$, Schutz $M$, Kuhner $D$, Bertsche $\mathrm{U}$, et al. The Intimin periplasmic domain mediates dimerisation and binding to peptidoglycan. Mol Microbiol. 2015;95(1):80-100.

137. Touze T, Hayward RD, Eswaran J, Leong JM, Koronakis V. Selfassociation of EPEC intimin mediated by the beta-barrel-containing anchor domain: a role in clustering of the Tir receptor. Mol Microbiol. 2004;51(1):73-87.

138. Leo JC, Oberhettinger P, Schutz M, Linke D. The inverse autotransporter family: intimin, invasin and related proteins. Int J Med Microbiol. 2015;305(2):276-82.

139. Andrews DL, Beames B, Summers MD, Park WD. Characterization of the lipid acyl hydrolase activity of the major potato (Solanum tuberosum) 
tuber protein, patatin, by cloning and abundant expression in a baculovirus vector. Biochem J. 1988;252(1):199-206.

140. Senda K, Yoshioka H, Doke N, Kawakita K. A cytosolic phospholipase A2 from potato tissues appears to be patatin. Plant Cell Physiol. 1996;37(3):347-53.

141. Strickland JA, Orr GL, Walsh TA. Inhibition of Diabrotica Larval Growth by Patatin, the Lipid Acyl Hydrolase from Potato Tubers. Plant Physiol. 1995;109(2):667-74.

142. Holk A, Rietz S, Zahn M, Quader H, Scherer GF. Molecular identification of cytosolic, patatin-related phospholipases A from Arabidopsis with potential functions in plant signal transduction. Plant Physiol. 2002;130(1):90-101.

143. Bárta J. BV. Patatin, the major protein of potato (Solanum tuberosum L.) tubers, and its occurrence as genotype effect: processing versus table potatoes. CJFS. 2008;26:347-59.

144. Hirschberg HJ, Simons JW, Dekker N, Egmond MR. Cloning, expression, purification and characterization of patatin, a novel phospholipase A. Eur J Biochem. 2001;268(19):5037-44.

145. Rydel TJ, Williams JM, Krieger E, Moshiri F, Stallings WC, Brown SM, et al. The crystal structure, mutagenesis, and activity studies reveal that patatin is a lipid acyl hydrolase with a Ser-Asp catalytic dyad. Biochemistry. 2003;42(22):6696-708.

146. Sato H, Frank DW, Hillard CJ, Feix JB, Pankhaniya RR, Moriyama K, et al. The mechanism of action of the Pseudomonas aeruginosa-encoded type III cytotoxin, ExoU. EMBO J. 2003;22(12):2959-69.

147. Phillips RM, Six DA, Dennis EA, Ghosh P. In vivo phospholipase activity of the Pseudomonas aeruginosa cytotoxin ExoU and protection of mammalian cells with phospholipase A2 inhibitors. J Biol Chem. 2003;278(42):41326-32.

148. Banerji S, Flieger A. Patatin-like proteins: a new family of lipolytic enzymes present in bacteria? Microbiology. 2004;150(Pt 3):522-5.

149. Arpigny JL, Jaeger KE. Bacterial lipolytic enzymes: classification and properties. Biochem J. 1999;343 Pt 1:177-83.

150. da Mata Madeira PV, Zouhir S, Basso P, Neves D, Laubier A, Salacha $\mathrm{R}$, et al. Structural Basis of Lipid Targeting and Destruction by the Type $\mathrm{V}$ Secretion System of Pseudomonas aeruginosa. J Mol Biol. 2016;428(9 Pt A):1790-803.

151. Casasanta MA, Yoo CC, Smith HB, Duncan AJ, Cochrane K, Varano $\mathrm{AC}$, et al. A chemical and biological toolbox for Type $\mathrm{Vd}$ secretion: 
Characterization of the phospholipase A1 autotransporter FplA from Fusobacterium nucleatum. J Biol Chem. 2017.

152. van Meer G, Voelker DR, Feigenson GW. Membrane lipids: where they are and how they behave. Nat Rev Mol Cell Biol. 2008;9(2):112-24. 153. Bretscher MS. Asymmetrical lipid bilayer structure for biological membranes. Nat New Biol. 1972;236(61):11-2.

154. Verkleij AJ, Zwaal RF, Roelofsen B, Comfurius P, Kastelijn D, van Deenen LL. The asymmetric distribution of phospholipids in the human red cell membrane. A combined study using phospholipases and freeze-etch electron microscopy. Biochim Biophys Acta. 1973;323(2):178-93.

155. Sohlenkamp C, Geiger O. Bacterial membrane lipids: diversity in structures and pathways. FEMS Microbiol Rev. 2016;40(1):133-59.

156. Marquardt D, Geier B, Pabst G. Asymmetric lipid membranes: towards more realistic model systems. Membranes (Basel). 2015;5(2):18096.

157. Devaux PF, Morris R. Transmembrane asymmetry and lateral domains in biological membranes. Traffic. 2004;5(4):241-6.

158. Daleke DL. Phospholipid flippases. J Biol Chem. 2007;282(2):821-5. 159. Op den Kamp JA. Lipid asymmetry in membranes. Annu Rev Biochem. 1979;48:47-71.

160. Lemmon MA. Membrane recognition by phospholipid-binding domains. Nat Rev Mol Cell Biol. 2008;9(2):99-111.

161. Watt SA, Kular G, Fleming IN, Downes CP, Lucocq JM. Subcellular localization of phosphatidylinositol 4,5-bisphosphate using the pleckstrin homology domain of phospholipase C delta1. Biochem J. 2002;363(Pt 3):657-66.

162. Shewan A, Eastburn DJ, Mostov K. Phosphoinositides in cell architecture. Cold Spring Harb Perspect Biol. 2011;3(8):a004796.

163. Berridge MJ. Inositol trisphosphate and calcium signalling. Nature. 1993;361(6410):315-25.

164. Sakai T, Sugiyama, T., Banno, Y., Kato, Y., Nozawa, Y. Involvement of phosphatidylcholine hydrolysis by phospholipase $\mathrm{C}$ in prostaglandin $\mathrm{F} 2 \mathrm{~A}$ induced 1,2-diacylglycerol formation in osteoblast-like MC3T3-E1 cells. J Bone Miner Metab. 2004;22: 198.

165. Shimanuki H. Diseases and pests of honey bees. In: Science and Education Administration USDoAW, DC, USA, editor. Bee Keeping in the United States. 3351980. p. 118-28.

166. Brennan M, Thomas DY, Whiteway M, Kavanagh K. Correlation between virulence of Candida albicans mutants in mice and Galleria mellonella larvae. FEMS Immunol Med Microbiol. 2002;34(2):153-7. 
167. Jander G, Rahme LG, Ausubel FM. Positive correlation between virulence of Pseudomonas aeruginosa mutants in mice and insects. J Bacteriol. 2000;182(13):3843-5.

168. Salamitou S, Ramisse F, Brehelin M, Bourguet D, Gilois N, Gominet $M$, et al. The plcR regulon is involved in the opportunistic properties of Bacillus thuringiensis and Bacillus cereus in mice and insects. Microbiology. 2000;146 ( Pt 11):2825-32.

169. Lionakis MS. Drosophila and Galleria insect model hosts: new tools for the study of fungal virulence, pharmacology and immunology. Virulence. 2011;2(6):521-7.

170. Hoffmann JA. Innate immunity of insects. Curr Opin Immunol. 1995;7(1):4-10.

171. Kavanagh K, Reeves EP. Exploiting the potential of insects for in vivo pathogenicity testing of microbial pathogens. FEMS Microbiol Rev. 2004;28(1):101-12.

172. Cotter G, Doyle S, Kavanagh K. Development of an insect model for the in vivo pathogenicity testing of yeasts. FEMS Immunol Med Microbiol. 2000;27(2):163-9.

173. Tsai CJ, Loh JM, Proft T. Galleria mellonella infection models for the study of bacterial diseases and for antimicrobial drug testing. Virulence. 2016;7(3):214-29.

174. Klein K, Sonnabend MS, Frank L, Leibiger K, Franz-Wachtel M, Macek B, et al. Deprivation of the Periplasmic Chaperone SurA Reduces Virulence and Restores Antibiotic Susceptibility of Multidrug-Resistant Pseudomonas aeruginosa. Front Microbiol. 2019;10:100.

175. Trunk T, Casasanta MA, Yoo CC, Slade DJ, Leo JC. Comparison of type $5 \mathrm{~d}$ autotransporter phospholipases demonstrates a correlation between high activity and intracellular pathogenic lifestyle. Biochem J. 2019;476(18):2657-76.

176. Ramarao N, Nielsen-Leroux C, Lereclus D. The insect Galleria mellonella as a powerful infection model to investigate bacterial pathogenesis. J Vis Exp. 2012(70):e4392.

177. Tamae C, Liu A, Kim K, Sitz D, Hong J, Becket E, et al. Determination of antibiotic hypersensitivity among 4,000 single-gene-knockout mutants of Escherichia coli. J Bacteriol. 2008;190(17):5981-8.

178. Tashiro Y, Sakai R, Toyofuku M, Sawada I, Nakajima-Kambe T, Uchiyama $\mathrm{H}$, et al. Outer membrane machinery and alginate synthesis regulators control membrane vesicle production in Pseudomonas aeruginosa. J Bacteriol. 2009;191(24):7509-19. 
179. Sydenham M, Douce G, Bowe F, Ahmed S, Chatfield S, Dougan G. Salmonella enterica serovar typhimurium surA mutants are attenuated and effective live oral vaccines. Infect Immun. 2000;68(3):1109-15.

180. Richmond GS, Smith TK. Phospholipases A(1). Int J Mol Sci. 2011;12(1):588-612.

181. Ghannoum MA. Potential role of phospholipases in virulence and fungal pathogenesis. Clin Microbiol Rev. 2000;13(1):122-43, table of contents.

182. Shimuta K, Ohnishi M, lyoda S, Gotoh N, Koizumi N, Watanabe H. The hemolytic and cytolytic activities of Serratia marcescens phospholipase A (PhIA) depend on lysophospholipid production by PhIA. BMC Microbiol. 2009;9:261.

183. Gonzalez-Bullon D, Martin C, Ostolaza H. Characterization of the Intrinsic Phospholipase A1 Activity of Bordetella pertussis Adenylate Cyclase Toxin. Toxins (Basel). 2018;10(12).

184. Flores-Diaz M, Monturiol-Gross L, Naylor C, Alape-Giron A, Flieger A. Bacterial Sphingomyelinases and Phospholipases as Virulence Factors. Microbiol Mol Biol Rev. 2016;80(3):597-628.

185. Hatlem D, Trunk T, Linke D, Leo JC. Catching a SPY: Using the SpyCatcher-SpyTag and Related Systems for Labeling and Localizing Bacterial Proteins. Int J Mol Sci. 2019;20(9). 

I 

OPEN ACCESS

Edited by:

Paolo Visca,

Università degli Studi Roma Tre, Italy

Reviewed by:

Paola Sperandeo,

University of Milan, Italy

Francesco Imperi,

Department of Science, Roma Tre

University, Italy

*Correspondence: Erwin Bohn

erwin.bohn@med.uni-tuebingen.de

tThese authors have contributed equally to this work

Specialty section: This article was submitted to Antimicrobials, Resistance and

Chemotherapy,

a section of the journal

Frontiers in Microbiology

Received: 28 November 2018

Accepted: 17 January 2019

Published: 21 February 2019

Citation:

Klein K, Sonnabend MS, Frank L, Leibiger K, Franz-Wachtel M,

Macek B. Trunk T, Leo JC Autenrieth IB, Schütz $M$ and Bohn E (2019) Deprivation of the Periplasmic Chaperone SurA Reduces Virulence and Restores Antibiotic Susceptibility of Multidrug-Resistant Pseudomonas aeruginosa. Front. Microbiol. 10:100

doi: 10.3389/fmicb.2019.00100

\section{Deprivation of the Periplasmic Chaperone SurA Reduces Virulence and Restores Antibiotic Susceptibility of Multidrug-Resistant Pseudomonas aeruginosa}

\author{
Kristina Klein ${ }^{1 \dagger}$, Michael S. Sonnabend ${ }^{1+}$, Lisa Frank ${ }^{1}$, Karolin Leibiger ${ }^{1}$, \\ Mirita Franz-Wachtel ${ }^{2}$, Boris Macek ${ }^{2}$, Thomas Trunk ${ }^{3}$, Jack C. Leo ${ }^{3}$, Ingo B. Autenrieth ${ }^{1}$, \\ Monika Schütz ${ }^{1}$ and Erwin Bohn ${ }^{1 *}$
}

\begin{abstract}
Interfakultäres Institut für Mikrobiologie und Infektionsmedizin Tübingen (IMIT), Institut für Medizinische Mikrobiologie und Hygiene, Universität Tübingen, Tübingen, Germany, ${ }^{2}$ Proteome Center Tübingen, Universität Tübingen, Tübingen, Germany,

${ }^{3}$ Section for Genetics and Evolutionary Biology, Department of Biosciences, University of Oslo, Oslo, Norway
\end{abstract}

Pseudomonas aeruginosa is one of the main causative agents of nosocomial infections and the spread of multidrug-resistant strains is rising. Therefore, novel strategies for therapy are urgently required. The outer membrane composition of Gram-negative pathogens and especially of $\mathrm{Pa}$ restricts the efficacy of antibiotic entry into the cell and determines virulence. For efficient outer membrane protein biogenesis, the $\beta$-barrel assembly machinery (BAM) complex in the outer membrane and periplasmic chaperones like Skp and SurA are crucial. Previous studies indicated that the importance of individual proteins involved in outer membrane protein biogenesis may vary between different Gram-negative species. In addition, since multidrug-resistant $P$ a strains pose a serious global threat, the interference with both virulence and antibiotic resistance by disturbing outer membrane protein biogenesis might be a new strategy to cope with this challenge. Therefore, deletion mutants of the non-essential BAM complex components bamB and bam $C$, of the skp homolog h/pA as well as a conditional mutant of surA were investigated. The most profound effects for both traits were associated with reduced levels of SurA, characterized by increased membrane permeability, enhanced sensitivity to antibiotic treatment and attenuation of virulence in a Galleria mellonella infection model. Strikingly, the depletion of SurA in a multidrug-resistant clinical bloodstream isolate re-sensitized the strain to antibiotic treatment. From our data we conclude that SurA of $P$ a serves as a promising target for developing a drug that shows antiinfective activity and re-sensitizes multidrug-resistant strains to antibiotics.

Keywords: SurA, Pseudomonas aeruginosa, virulence, multidrug resistance, antibiotics, outer membrane protein biogenesis 


\section{INTRODUCTION}

The widespread use of antibiotics is causative for the rapid development of multidrug-resistant strains. Particularly, the emergence of carbapenem-resistant bacteria poses a significant threat to public health (Pendleton et al., 2013). The Gramnegative, opportunistic pathogen Pseudomonas aeruginosa $(\mathrm{Pa})$ belongs to the so-called ESKAPE group, comprising a group of the most common and multidrug-resistant bacteria (Rice, 2008). $P a$ can cause infections in a wide range of animal and plant hosts and is a leading cause of nosocomial infections, which are almost exclusively found in immunocompromised hosts (Lyczak et al., 2000; Lister et al., 2009). Pa displays numerous intrinsic and acquired resistance mechanism against antibiotics: (i) enzymatic and mutational resistance mechanisms like the production of $\beta$-lactamases, (ii) overexpression of efflux systems, and (iii) the low permeability of the outer membrane $(\mathrm{OM})$ that limits the penetration of antibiotic molecules (Yoshimura and Nikaido, 1982).

The major challenge for drugs against $\mathrm{Pa}$ and Gram-negative bacteria in general is to pass the bacterial OM. The OM provides a highly effective barrier against foreign and harmful molecules, allows import and export of essential substances such as nutrients and iron, is necessary for communication and harbors many virulence factors. The outer leaflet of the $\mathrm{OM}$ is constituted mainly by lipopolysaccharides (LPS), whereas the inner leaflet consists of phospholipids. This bilayer houses a great variety of outer membrane proteins (OMPs) that facilitate transport and other essential functions, and act as virulence factors (Nikaido, 2003). Many OMPs are porins and autotransporters. Both comprise a $\beta$-barrel domain and either facilitate transport of molecules across the OM (Chevalier et al., 2017) or can form cell surface exposed moieties that shape the interaction with the host and the extracellular environment (Leyton et al., 2012). For the insertion of these $\beta$-barrel proteins, Gram-negative bacteria employ a conserved transport system consisting of the periplasmic chaperones SurA, Skp, and DegP, which protect and guide newly synthesized proteins from the Sec translocon in the inner membrane to the $\mathrm{OM}$ and the $\beta$-barrel assembly machinery (BAM) complex (Sklar et al., 2007; Tashiro et al., 2009; Goemans et al., 2014; Li et al., 2018). Both SurA and Skp act as chaperones and are thought to form a partially redundant network. The importance of SurA and Skp for the OMP biogenesis is controversially discussed. At least in Escherichia coli (Ec) and Yersinia enterocolitica (Ye), SurA plays the major and Skp a less prominent role in folding and assembly of OMPs (Sklar et al., 2007; Volokhina et al., 2011; Weirich et al., 2017). However, in Neisseria mengitidis, Skp is more important for shaping the OMP composition than SurA, indicating species-specific differences (Tamae et al., 2008).

The BAM complex, which inserts the $\beta$-barrel proteins into the OM, consists of the central component BamA and the four lipoproteins BamB, BamC, BamD, and BamE (Noinaj et al., 2017). Of these subunits, only BamA and BamD are essential in most of the so far investigated Gramnegative bacteria, except Borrelia burgdorferi and Salmonella enterica (Wu et al., 2005; Malinverni et al., 2006; Fardini et al., 2009; Dunn et al., 2015). BamA is a $\beta$-barrel protein itself (Noinaj et al., 2017). Its C-terminal $\beta$-barrel domain is connected to an $\mathrm{N}$-terminal periplasmic domain which consists of five polypeptide transport-associated (POTRA) domains. The POTRA domains form several interactions with the other Bam subunits, building up the BAM complex and interact with both substrates and periplasmic chaperones such as SurA (Gu et al., 2016). BamB directly binds to the POTRA domains 2-5 of BamA and supports the stabilization of nascent OMPs by binding and delivering OMP $\beta$-strands to BamA (Heuck et al., 2011).

In $E c$, the deletion of one of the non-essential BAM complex components or the related periplasmic shuttle protein SurA may lead to an altered protein composition in the $\mathrm{OM}$ and/or disturbed $\mathrm{OM}$ integrity and therefore to a higher susceptibility to various antibiotics (Behrens et al., 2001; Onufryk et al., 2005). Using $Y e$ we have previously shown that the deletion of surA and bamB implies a significantly decreased virulence and more efficient clearance of $Y e$ infection by the host in vivo (Weirich et al., 2017).

In $\mathrm{Pa}, \mathrm{BamA}$, and the BamE-homolog OmlA have already been recognized to play a role in the stability of the OM and susceptibility to environmental stress (Ochsner et al., 1999; Yorgey et al., 2001; Hoang et al., 2011). For BamB of Pa, an enhanced susceptibility against lysozyme and cell wall targeting antibiotics as well as a decreased growth in vivo have been demonstrated recently (Lee et al., 2017).

Thus, it is well recognized that the BAM complex itself as well as chaperones in delivering proteins to the outer membrane are critical for membrane integrity as well as antibiotic resistance and could therefore be targets for drug development (Tamae et al., 2008; Weirich et al., 2017; Storek et al., 2018; Vij et al., 2018). Nevertheless, previous studies revealed species-specific differences in the importance of individual components in OMP biogenesis such as Skp and SurA (Sklar et al., 2007; Volokhina et al., 2011; Weirich et al., 2017). In addition, for considering such proteins as targets for $\mathrm{Pa}$ it would be mandatory to affect multidrug-resistant strains and break resistance against commonly used antibiotics.

To identify potential targets in order to possibly develop new strategies to treat especially infections caused by multidrugresistant $P a$, we investigated the role of components involved in the assembly of proteins into the $\mathrm{OM}$ by deletion of the non-essential BAM complex components BamB and a BamC homolog as well as the periplasmic shuttle proteins SurA and HlpA (a Skp-like protein) in Pa PA14. Depletion of SurA had the greatest impact on $\mathrm{OM}$ integrity and caused profound changes in the protein composition of the OM. These changes broadened the spectrum of antibiotics that could be used for treatment of $\mathrm{Pa}$ infection, and they lowered the minimum inhibitory concentration of clinically important antibiotics. Additionally, depletion of SurA enhanced clearance of $\mathrm{Pa}$ infection by the host. Taken together, our findings indicate that specifically SurA could serve as a novel antivirulence and/or resistance-breaking target even in multidrug-resistant strains of $\mathrm{Pa}$. 


\section{MATERIALS AND METHODS}

\section{Bacterial Strains and Culture Conditions}

Bacterial strains and plasmids used in this study are listed in Table S1. Bacteria were cultivated overnight at $37^{\circ} \mathrm{C}$ with shaking at $200 \mathrm{rpm}$ in lysogeny broth (LB) containing suitable antibiotics but without any additives such as arabinose, if not otherwise stated. Antibiotics were added at the following concentrations: Tetracycline (Tet; AppliChem \#A2228) $15 \mu \mathrm{g} / \mathrm{ml}$, ampicillin (Amp; AppliChem \#A0839) $100 \mu \mathrm{g} / \mathrm{ml}$ and gentamicin (Gm; AppliChem \#A1492) $15 \mu \mathrm{g} / \mathrm{ml}$ (Ec strains) or $75 \mu \mathrm{g} / \mathrm{ml}$ for $\mathrm{Gm}$ and $50 \mu \mathrm{g} / \mathrm{ml}$ for Tet ( $\mathrm{Pa}$ strains). If not stated otherwise, overnight cultures were diluted 1:20 into fresh LB medium containing suitable antibiotics (and/or additives like $0.2 \%$ arabinose (Sigma Aldrich \#A3256) for the conditional surA mutant) and grown for $3 \mathrm{~h}$ at $37^{\circ} \mathrm{C}$ and $200 \mathrm{rpm}$ to obtain subcultures in exponential phase $\left(\mathrm{OD}_{600}=0.5\right)$. The growth of bacteria in $\mathrm{LB}$ at $37^{\circ} \mathrm{C}$ in a 24 -well-plate was measured using Tecan Infinite ${ }^{\circledR} 200 \mathrm{PRO}$ at $37^{\circ} \mathrm{C}$. To investigate the growth under iron-restricted conditions, indicated concentrations of 2,2'-Bipyridyl (Sigma Aldrich \#D216305) were added.

\section{Generation of In-frame Deletion Mutants}

In-frame deletion mutants were generated using the suicide plasmid pEXG2 (Rietsch et al., 2005). The primers used in this study are listed in Table S2. First, the flanking regions (consisting of $30 \mathrm{bp}$ at the $3^{\prime}$ end and $30 \mathrm{bp}$ at the $5^{\prime}$ end of the gene of interest plus $\sim 800 \mathrm{bp}$ for each flanking region) and a pEXG2 fragment were amplified by PCR and ligated using Gibson assembly (Gibson, 2009). In general, constructed plasmids were verified by DNA sequencing, transformed into Ec SM10 $\lambda$ pir and subsequently mobilized by conjugation into PA14. Merodiploids were selected on LB agar plates containing irgasan $(25 \mu \mathrm{g} / \mathrm{ml}$; Sigma Aldrich \#72779) and $\mathrm{Gm}(75 \mu \mathrm{g} / \mathrm{ml})$. To achieve the second cross-over, counter selection on no-salt lysogeny broth (NSLB) agar containing 15\% sucrose was performed (Sigma Aldrich \#S7903). Finally, the loss of the plasmid was tested by streaking colonies on LB agar plates containing $\mathrm{Gm}(75 \mu \mathrm{g} / \mathrm{ml})$ and in parallel on LB agar plates without antibiotics. In-frame deletion mutants were confirmed by PCR using (i) a primer pair flanking the target gene and (ii) a primer pair where one primer binds to the coding region of the target gene.

\section{Generation of Conditional Depletion Mutants}

As stated also in the results section, we were not able to create an in-frame surA deletion mutant. Therefore, a conditional mutant was generated, starting from a merodiploid PA14::pEXG2-surA clone. For the integration of exogenous surA, the plasmid mini-

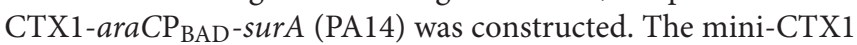
(Hoang et al., 2000) is an optimized self-proficient integration vector for $\mathrm{Pa}$ containing a $\varphi \mathrm{CTX}$ attachment site for integration of foreign genes into the chromosome. The coding sequence (cds) of tolB of the vector mini-CTX1-araCP $\mathrm{BAD}_{\mathrm{BAD}}$-tolB (Lo Sciuto et al., 2014) was replaced by the cds of surA using PCR amplification and Gibson assembly. The mini-CTX1-araCP $\mathrm{BAD}^{-}$ surA construct was integrated into the attB neutral site of the chromosome of PA14::pEXG2-surA as described recently (Hoang et al., 2000; Lo Sciuto et al., 2014) in the presence of Tet $(50 \mu \mathrm{g} / \mathrm{ml}), \mathrm{Gm}(100 \mu \mathrm{g} / \mathrm{ml})$ and arabinose $(0.2 \%)$. Afterwards, the endogenous copy of the surA gene was deleted in-frame under SurA-inducing conditions and confirmed as described above. Excision of the mini-CTX1 backbone containing the Tet resistance cassette was performed using Flp recombinase as described (Hoang et al., 2000) and verified by PCR. Likewise, a conditional surA mutant of the clinical $\mathrm{Pa}$ isolate ID72 was

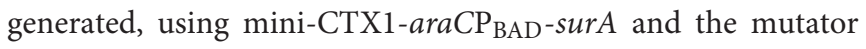
plasmid pEXG2-surA ID72. For the complementation of bamB, the mini-CTX1 vector was used to introduce an arabinoseinducible copy of bamB into the genome of PA14 bamB as described for surA.

\section{Electron Microscopy}

A total of $5 \times 10^{9}$ bacteria were harvested and fixed in Karnovsky's fixative, embedded in agarose, cut in small blocks and fixed again in Karnovsky's fixative. After post-fixation and embedding in glycid ether, blocks were cut using an ultramicrotome. Sections $(30 \mathrm{~nm})$ were mounted on copper grids and analyzed using a Zeiss LIBRA transmission electron microscope.

\section{Generation of Overexpression Plasmids for Protein Purification}

The cds of PA14 surA was subcloned into the vector pTXB1, resulting in pTXB1-surA-Intein. pET28a-bamB-His 6 was generated by Genscript Inc. Both plasmids were transformed into Ec BL21 (DE3) (Invitrogen \#C600003). The sequence encoding full-length $p l p D$ from $P a$ PAO1 was synthesized with $E c$ codon optimization (ThermoFisher Scientific). The region coding for the passenger and the POTRA domain (residues 18-406) were subcloned into the expression vector pET28a+ (Novagen \#69864) using Gibson assembly with mutations leading to an inactive lipase and encoding a C-terminal hexa-histidine tag resulting in pET28a-plpD S60A/D207NHis (Liu and Naismith, 2008).

\section{Protein Purification and Generation of Polyclonal Antibodies}

For purification of SurA, Ec BL21 (DE3) harboring pTXB1surA-Intein was grown to an $\mathrm{OD}_{600}$ of 0.4 , induced by the addition of $100 \mu \mathrm{M}$ IPTG (Peqlab \#37-2020) and grown for another $4 \mathrm{~h}$ at $37^{\circ} \mathrm{C}$. Protein purification was performed using the IMPACT ${ }^{\mathrm{TM}}$ kit (New England Biolab \#E6901S) according to the manufacturer's instructions with subsequent size-exclusion chromatography on a HiLoad ${ }^{\mathrm{TM}}$ 16/600 Superdex ${ }^{\mathrm{TM}} 200$ pg column (GE Lifesciences). Fractions containing purified SurA were pooled, concentrated and validated by SDS-PAGE. For purification of BamB, Ec BL21 (DE3) harboring pTXB1-bamBHis 6 were grown to an $\mathrm{OD}_{600}$ of 0.6 , induced by the addition of $100 \mu \mathrm{M}$ IPTG and grown overnight at $37^{\circ} \mathrm{C}$. Bacteria were pelleted and resuspended in buffer A [40 $\mathrm{mM}$ HEPES (Carl Roth \#9105.4), pH 7.4; $150 \mathrm{mM} \mathrm{NaCl}$ (VWR Chemicals \#27810.295)] following an incubation under stirring for $20 \mathrm{~min}$ at $4^{\circ} \mathrm{C}$ with $10 \mathrm{mM} \mathrm{MgSO}_{4}$ (AppliChem \#A6414), $20 \mathrm{mg} / \mathrm{ml}$ lysozyme (Sigma Aldrich \#6876), protease inhibitor tablets 
(Sigma Aldrich \#S8830) and a pinch of DNase (Sigma Aldrich \#DN25). Subsequently, bacteria were lysed using a French pressure cell, followed by sequential centrifugation steps at $4{ }^{\circ} \mathrm{C}(4,500 \times g, 15 \mathrm{~min} ; 20,000 \times g, 20 \mathrm{~min} ; 40,000 \times g$, $1 \mathrm{~h})$. Finally, the sterile-filtered $(0.2 \mu \mathrm{m}$ filter, Sarstedt $) \mathrm{His}_{6}{ }^{-}$ tagged protein was subjected to metal affinity chromatography (HisTrap $^{\text {TM }}$ HP, $5 \mathrm{ml}$, GE Life Sciences) and concentrated. Antibodies were raised in 2 rabbits each for SurA or BamB-His 6 and subsequently affinity-purified against purified SurA or BamB protein, respectively (Eurogentec).

For purification of PlpD lipase + POTRA domains, Ec BL21 Gold (DE3) cells (Agilent Technologies \#230132) harboring pET28a-plpD S60A/D207N-His were grown in autoinducing ZYP-5052 medium (Studier, 2005) at $30^{\circ} \mathrm{C}$, harvested $24 \mathrm{~h}$ post-inoculation by centrifugation and resuspended in running buffer containing $40 \mathrm{mM}$ sodium phosphate (Carl Roth \#K300.1), $400 \mathrm{mM} \mathrm{NaCl}$ and $20 \mathrm{mM}$ imidazole, pH 8.0 (AppliChem \#A1073). For lysis, additional EDTA-free protease inhibitor, $1 \mathrm{mM} \mathrm{MgCl} 2$ (Sigma Aldrich \#M8266), $1 \mathrm{mM} \mathrm{MnCl} 2$ (Merck \#8059300100), $0.1 \mathrm{mg} / \mathrm{ml}$ lysozyme and a pinch of DNase were added to the buffer before application to a French pressure cell. After centrifugation at $20,000 \times g$ and $4{ }^{\circ} \mathrm{C}$ for $35 \mathrm{~min}$, the sterile-filtered supernatant containing the $\mathrm{His}_{6}$-tagged protein was applied to a HisTrap ${ }^{\mathrm{Tm}} \mathrm{FF}$ column (GE Healthcare) and purified on an NGC Chromatography System (Bio-Rad). The protein was eluted from the column using a gradient of imidazole (to $0.5 \mathrm{M}$ ) and further purified on a HiPrep 26/60 Sephacryl S200 HR size exclusion column (GE Healthcare, USA) using $20 \mathrm{mM}$ Tris and $300 \mathrm{mM} \mathrm{NaCl}$ at $\mathrm{pH}$ 7.5. The production of antibodies was performed at the Section for Experimental Biomedicine (University of Life Sciences, Oslo, Norway) with license of the Norwegian Animal Research Authority (NARA) (http://www. mattilsynet.no/dyr_og_dyrehold/dyrevelferd/forsoksdyr/).

\section{NPN Assay}

To determine changes in the OM permeability of the generated mutants, the fluorescent, hydrophobic 1-Nphenylnaphthylamine (NPN) (Acros organics \#90-30-2) was used as described (Konovalova et al., 2016). Subcultured bacteria were washed and adjusted to an $\mathrm{OD}_{600}$ of 0.5 in $5 \mathrm{mM}$ HEPES buffer ( $\mathrm{pH}$ 7.2). NPN was added to the bacteria to a final concentration of $10 \mu \mathrm{M} .200 \mu \mathrm{l}$ of the bacterial suspension were transferred to 96-well F-bottom, black, nonbinding plates (Greiner Bio-one \#89089-582). Subsequently, fluorescence (excitation and emission wavelengths 350 and $420 \mathrm{~nm}$, respectively) was measured using the Tecan Infinite ${ }^{\circledR}$ 200 PRO. Polymyxin B (PMB, Merck \#A 231-40) served as a positive control and was added to a final concentration of $8 \mu \mathrm{g} / \mathrm{ml}$. Values obtained for a buffer-only control were subtracted from all values.

\section{Bile Salt Assay}

To analyze the sensitivity to bile salts, $10^{7}$ bacteria per well were inoculated in duplicates into a 24 well microtiter plate containing either $1 \mathrm{ml} \mathrm{LB}$ or $1 \mathrm{ml} \mathrm{LB}+0.3 \%$ bile salts (Sigma Aldrich \#B8756). The conditional surA mutant was additionally supplemented with $0.2 \%$ arabinose. The plate was incubated at $37^{\circ} \mathrm{C}$ and shaking at $160 \mathrm{rpm}$ for $8 \mathrm{~h}$ and $\mathrm{OD}_{600}$ was determined using the Tecan Infinite ${ }^{\circledR} 200$ PRO.

\section{Western Blot Analysis}

$5 \times 10^{8}$ bacteria per $\mathrm{ml}$ of subcultures grown for $3 \mathrm{~h}$ were boiled in $2.5 \times$ Laemmli buffer (Bio-Rad \#161-0747) containing $50 \mathrm{mM}$ DTT (Thermo Fisher Scientific \#R0861) at $95^{\circ} \mathrm{C}$ for $10 \mathrm{~min}$. SDS-PAGE was performed with $5 \times 10^{6}$ bacteria per lane using a $10 \%$ Mini-PROTEAN ${ }^{\circledR}$ TGX ${ }^{\mathrm{TM}}$ Precast Protein gel (BioRad). Subsequently, proteins were transferred to a nitrocellulose membrane. After blocking in 5\% skim milk in TBS (10 mM TrisHCL (Sigma \#T1503), $150 \mathrm{mM} \mathrm{NaCl}$; pH 7.6), the membrane was incubated with the primary antibody [rabbit anti-SurA, 1:200; rabbit anti-BamB-His 6 , 1:200; rabbit anti-OprD (kindly provided by Thilo Köhler, University of Geneva; Epp et al., 2001), 1:2,000; rabbit anti-PlpD serum 1:10,000; rabbit anti-RpoB $(E c), 1: 2,000$ (Abcam \#mAb EPR18704)] and afterwards with the secondary antibody (horseradish-peroxidase-conjugated goat anti-rabbit antibody 1:5,000, Thermo Fisher Scientific \#31460). Clarity $^{\mathrm{TM}}$ Western ECL Substrate (Bio-Rad \#170-5061) was added and signals were detected using a Fusion Solo $\mathrm{S}$ imager (Vilber). Protein bands were quantified via ImageJ. In contrast to SurA and OprD, where RpoB was used as a loading control for quantification, for PlpD the unspecific band of $\sim 75 \mathrm{kDa}$ served as a loading control.

\section{Enrichment of OM Fractions}

Preparation of the OM was conducted as described (Thein et al., 2010; Oberhettinger et al., 2015; Weirich et al., 2017). In short, PA14 strains including the conditional surA mutant were grown overnight in LB. Subcultures (1:20 dilution) were then grown in $\mathrm{LB}$ to an $\mathrm{OD}_{600}$ of $0.5-0.7$. For complementation of the conditional surA mutant $0.2 \%$ arabinose was added in the subculture. After centrifugation, $2.5 \times 10^{10}$ bacteria were resuspended in $0.5 \mathrm{ml}$ of resuspension buffer $(0.2 \mathrm{M}$ Tris, $1 \mathrm{M}$ sucrose, $1 \mathrm{mM}$ EDTA (Applichem \#A5097), $\mathrm{pH} 8.0$ ), then $5,000 \mathrm{U}$ lysozyme were added and incubated for $5 \mathrm{~min}$ at room temperature. Subsequently, $3.2 \mathrm{ml} \mathrm{H}_{2} \mathrm{O}$ were added and incubated for $20 \mathrm{~min}$ at room temperature until spheroplasts were formed. Then, $5 \mathrm{ml}$ of extraction buffer (2\% Triton X100 (AppliChem \#A4975), $50 \mathrm{mM}$ Tris, $10 \mathrm{mM} \mathrm{MgCl}_{2}, \mathrm{pH}$ 8.0) together with $5 \mu$ l DNase I (Roche Applied Science $\# 03539121103$ ) were added and incubated on a rotator for $20 \mathrm{~min}$ at room temperature to solubilize the inner membrane fraction with Triton X-100 (Schnaitman, 1971; Page and Taylor, 1988). The lysate was centrifuged at $85,000 \times g$ for $1 \mathrm{~h}$ at $4^{\circ} \mathrm{C}$ and the pellet containing the $\mathrm{OM}$ fraction was washed three times in $2.5 \mathrm{ml} \mathrm{H}_{2} \mathrm{O}$ by centrifugation at $292,000 \times \mathrm{g}$ for $15 \mathrm{~min}$ at $4^{\circ} \mathrm{C}$. The pellet containing the $\mathrm{OM}$ fraction was resuspended in $300 \mu \mathrm{l} \mathrm{H}_{2} \mathrm{O}$.

\section{NanoLC-MS/MS Analysis and Data Processing}

The protein concentration of the OM samples was measured using the Pierce ${ }^{\mathrm{TM}}$ BCA Protein Assay Kit (Thermo Fisher Scientific \#23225). $10 \mu \mathrm{g}$ of each sample was subjected to SDSPAGE and stained with Roti ${ }^{\circledR}$-Blue Colloidal Coomassie Staining 
Solution. OM fractions were analyzed as described previously (Weirich et al., 2017) with slight modification: Coomassiestained gel pieces were digested in-gel with trypsin (Borchert et al., 2010), and desalted peptide mixtures (Rappsilber et al., 2007) were separated on an Easy-nLC 1200 (Thermo Scientific) system coupled to an LTQ Orbitrap Elite mass spectrometer (Thermo Scientific). The peptide mixtures were injected onto the column in HPLC solvent A ( $0.1 \%$ formic acid) at a flow rate of $500 \mathrm{nl} / \mathrm{min}$ and subsequently eluted with an $127 \mathrm{~min}$ segmented gradient of $5-33-50-90 \%$ of HPLC solvent B (80\% acetonitrile in $0.1 \%$ formic acid) at a flow rate of $200 \mathrm{nl} / \mathrm{min}$. The mass spectrometer was operated in positive ion mode, and spectra were recorded in a mass range from $\mathrm{m} / \mathrm{z} 300$ to 2000 with a resolution of 120,000 . The 15 most intense ions were sequentially isolated and fragmented in the linear ion trap using collision-induced dissociation (CID) and default CID settings. The target values for MS scans and MS/ MS fragmentation were $10^{6}$ and 5,000 charges, respectively. Sequenced precursor masses were excluded from further selection for $60 \mathrm{~s}$.

Acquired MS spectra were processed with MaxQuant software package version 1.5.2.8 (Cox and Mann, 2008) with integrated Andromeda search engine (Elias and Gygi, 2007). Database search was performed against a target-decoy $\mathrm{Pa}$ UCBPP-PA14 database obtained from Uniprot, containing 5886 protein entries, and 285 commonly observed contaminants. Endoprotease trypsin was defined as protease with a maximum of two missed cleavages. Oxidation of methionine and $\mathrm{N}$ terminal acetylation were specified as variable modifications, and carbamidomethylation on cysteine was set as fixed modification. Initial maximum allowed mass tolerance was set to $4.5 \mathrm{ppm}$ (for the survey scan) and 0.5 Da for CID fragment ions. Peptide, protein and modification site identifications were reported at a false discovery rate (FDR) of 0.01 , estimated by the target/decoy approach (Elias and Gygi, 2007). The label-free algorithm was enabled, as was the "match between runs" option (Luber et al., 2010). The detection limit was calculated as the mean of the lowest label-free quantification (LFQ) values of each sample. Multiple $t$-tests were performed and FDR of differences in the $\log _{2}$ protein amount between mutant and wild type (WT) were assessed using the two-stage step-up method (Benjamini et al., 2006) with GraphPad Prism 7.04 software. Differences in protein amount with a FDR $<0.1$ were considered significant.

The mass spectrometry proteomics data have been deposited to the ProteomeXchange Consortium via the PRIDE (Vizcaino et al., 2016) partner repository with the dataset identifier PXD011849 (Username: reviewer54276@ebi.ac.uk, Password: i3rXLDrr).

\section{RNA Isolation and qRT-PCR}

$5 \times 10^{9}$ bacteria grown as described for the mass spectrometry analyses were resuspended in $1 \mathrm{ml} \mathrm{TRIzol}{ }^{\mathrm{TM}}$ Reagent (Thermo Fisher Scientific \#15596018). RNA isolation and DNase digestion were conducted as described previously (Goerke et al., 2000; Münzenmayer et al., 2016). The RNA $(0.1 \mu \mathrm{g} / \mu \mathrm{l}$ in RNA storage solution, Invitrogen \#AM7000) was diluted 1:10 with RNase-free water (Ambion \#AM9937). To exclude samples with detectable DNA contamination, a quantitative PCR using the QuantiFast
SYBR Green PCR Kit (Qiagen \# 204054) for the house keeping gene gyrB was performed. mRNA expression was assessed by quantitative RT-PCR using the QuantiFast SYBR Green qRTPCR Kit (Qiagen \# 204154) according to the manufacturer. A standard curve was generated by a serial dilution of one sample. Efficiency of the PCR and Cp values were calculated with the help of LightCycler480 software (Roche). Relative quantification was conducted as described by Pfaffl (Pfaffl, 2001). The used primers are listed in Table S2.

\section{Serum Killing Assay}

A serum killing assay was performed using the BacTiter-Glo ${ }^{\mathrm{TM}}$ Microbial Cell Viability Assay (Promega) as described (Necchi et al., 2017) with slight modifications. Normal human serum (NHS) from healthy donors (Transfusion medicine, University hospital Tübingen) was stored in aliquots at $-80^{\circ} \mathrm{C}$. Heat inactivated serum (HIS) was generated by incubating the serum at $56^{\circ} \mathrm{C}$ for $30 \mathrm{~min}$ immediately before use. $5 \times 10^{6}$ bacteria were incubated at $37^{\circ} \mathrm{C}$ in $100 \mu \mathrm{l} 10 \%$ HIS- or $10 \%$ NHS-PBS in a 96 well V-bottom microtiter plate (Greiner bio-one \#651101) in triplicates for various time periods. After that, plates were centrifuged at $3,500 \times g$ for $5 \mathrm{~min}$ and the pelleted bacteria were resuspended in $100 \mu \mathrm{l}$ PBS (Gibco $\left.{ }^{\mathrm{TM}} \# 14040-091\right)$. To determine the number of viable bacterial cells, $50 \mu \mathrm{l}$ bacterial suspension and $50 \mu \mathrm{l} \mathrm{BacTiter-Glo}{ }^{\mathrm{TM}}$ reagent (Promega \#G8321) were transferred to a white lumitrac 96 well F-bottom microtiter plate (Greiner bio-one \#655075) and the ATP levels inside the bacteria were quantified with a Tecan Infinite ${ }^{\circledR} 200$ PRO.

\section{Galleria mellonella Infection Model}

Galleria mellonella (TruLarv ${ }^{\mathrm{TM}}$ ) larvae were purchased from Biosystems Technology. Subcultured bacteria were serially diluted to $10^{3} / \mathrm{ml}$ in PBS. Each G. mellonella larva was injected with $10 \mu \mathrm{l}$ of $10^{3} / \mathrm{ml}$ bacterial dilution using a 30 gauge syringe (BD Biosciences). The larvae were then incubated at $37^{\circ} \mathrm{C}$ and monitored for 3 days after infection. Larvae were considered dead when no movement could be triggered by touching the larvae with a forceps. Ten microliter aliquots of the bacterial dilutions injected into the larvae were plated in triplicates on LB agar plates and the CFU was determined. The mean administered bacterial dose for all experiments was $12 \pm 2$ bacteria.

\section{Antibiotic Susceptibility Testing}

For determination of antibiotic susceptibility, bacterial strains were grown at $37^{\circ} \mathrm{C}$ overnight. Physiological sodium chloride solution was inoculated to a McFarland standard of 0.5. From this solution, bacteria were streaked with cotton swabs onto Mueller-Hinton agar plates with or without $0.2 \%$ arabinose. E-tests (Liofilchem) were conducted according to CLSI standard protocols to test the sensitivity of the different strains for the following antibiotics: ampicillin/sulbactam (\#92070); piperacillin/ tazobactam (\#92108); ticarcillin/ clavulanic acid (\#921171); doripenem (\#92040); meropenem (\#920840); cefotaxime (\#920061); cefepime (\#921271); ceftazidime (\#921380); levofloxacin (\#92081); ciprofloxacin (\#920450); fosfomycin (\#920790); vancomycin (\#920570); erythromycin (\#92051); trimethoprim/ sulfamethoxazole (\#921231). 


\section{Statistics}

Statistics were performed using GraphPad Prism 7.04 software as described for each experiment in the table or figure legends.

\section{RESULTS}

\section{Generation of Pa Strains Carrying Deletions for BAM Complex Components and Periplasmic Chaperones}

The BAM complex and associated chaperones may be interesting targets for developing novel drugs against Gram-negative bacteria. Their inhibition could possibly re-sensitize Gramnegative pathogens to antibiotics to which they are resistant or enable the use of antibiotics typically not being able to cross the $\mathrm{OM}$ barrier and thus not applicable for treatment of infection with Gram-negative pathogens (e.g., vancomycin) (Sydenham et al., 2000; Rolhion et al., 2005; Fardini et al., 2009; Weirich et al., 2017). Because of the clinical importance and increasing numbers of multidrug-resistant strains we addressed the role of $\mathrm{Pa} \mathrm{BamB}$ (PA14_14910), BamC (PA14_51260), the Skp-like protein HlpA (PA14_17170), and SurA (PA14_07760) for fitness and virulence of $\mathrm{Pa}$ in order to determine which factors might be the best targets for drug development. For this purpose we generated single gene deletions, which were verified by PCR using genomic DNA as template. Mass spectrometry analyses of OM fractions (typically highly contaminated with cytoplasmic proteins) of the $b a m B$, bam $C$, and hlpA deletion strains compared to wild type (WT) revealed the absence of the corresponding proteins (highlighted in boldface in Table S3B).

Although we initiated numerous attempts, we were not able to generate a surA deletion mutant. As an alternative, we created a stable and unmarked PA14 surA conditional mutant harboring an arabinose-inducible copy of the surA coding sequence, resulting in the conditional surA mutant $\triangle$ surA araC- $\mathrm{P}_{\mathrm{BAD}}$-surA (Figure 1A), for convenience termed surA. Complementation of $\operatorname{sur} A$ was achieved by the addition of $0.2 \%$ arabinose to the culture media where appropriate (termed surA SurA+). To check for expression of surA, mRNA levels were determined by quantitative RT-PCR, using gyrB as a housekeeping gene (Table S4). The relative number of mRNA transcripts of the conditional surA mutant grown in the absence of arabinose was reduced by $92 \%$ compared to bacteria harvested after growth in the presence of arabinose (surA SurA+). Therefore, in the absence of arabinose surA is still expressed in a low amount because the araC- $\mathrm{P}_{\mathrm{BAD}}$ promoter is leaky and cannot be repressed by catabolite repression (Meisner and Goldberg, 2016). In addition, we assessed the presence of SurA protein in whole cell lysates by Western blot analysis (Figure 1B). Using the conditional surA mutant, SurA protein could not be detected after growth in the absence of arabinose indicating a SurA protein level below the detection limit of the Western blot analysis, while production of SurA was restored in the presence of arabinose. Growth of the (conditional) mutants was investigated at $37^{\circ} \mathrm{C}$ in LB medium (Figure 1C). Only a slight but significant reduction in growth $(p<0.01)$ was observed between $6 \mathrm{~h}$ and $12 \mathrm{~h}$ after start of the experiment for the conditional surA mutant, while all other mutants grew comparably to the PA14 WT strain.

\section{SurA and BamB Are Important for OM Integrity}

Integrity of the $\mathrm{OM}$ is a pivotal feature of Gram-negative bacteria mediating protection against drugs and harsh environments including mucosal surfaces with antimicrobial peptide production. Since SurA delivers OMPs to the OM, where they are inserted by the BAM complex, an inhibition of parts of this pathway should result in an altered OM composition and possibly a reduced $\mathrm{OM}$ integrity. To evaluate changes in $\mathrm{OM}$ integrity induced by SurA depletion, or bamB, bamC or hlpA deletion, we first performed a 1-N-phenylnaphthylamine (NPN) assay. NPN fluoresces only in hydrophobic environments. Thus, if the integrity of the OM is compromised in one of the mutant strains, NPN can reach the phospholipid bilayer of the inner OM leaflet more efficiently (Konovalova et al., 2016). Higher fluorescence values therefore indicate a reduced OM integrity. It was shown previously that disturbance of the OM by polymyxin $\mathrm{B}$ (PMB) leads to a strong and significant increase of NPN fluorescence. Therefore, $\mathrm{PMB}$ was used as a positive control in our assay (Figure 2A). We found that the depletion of SurA, but not the deletion of bamB, bamC or $h l p A$ led to a significant increase of fluorescence, compared to the wildtype strain (WT). This means that only the depletion of SurA significantly enhances the entry of NPN. The complementation of surA by growing the strain in the presence of arabinose (surA SurA+) resulted in a NPN fluorescence signal comparable to that of PA14 WT, indicating that the phenotype can be fully restored by the complementation.

Next we investigated the susceptibility to bile salts, which act as physiological detergents in the intestinal tract (Merritt and Donaldson, 2009). Treatment with $0.3 \%$ bile salts significantly reduced the growth of the (conditional) $\operatorname{sur} A, \operatorname{bamB}$, and $\operatorname{bamC}$ mutants, but not of the hlpA mutant or surA SurA+ (Figure 2B). For complementation of the $b a m B$ deletion mutant, a mini-CTX1 plasmid expressing bamB under the control of an arabinoseinducible promoter was introduced and induced with $0.2 \%$ arabinose (bamB BamB +$)$.

\section{Depletion of SurA and BamB Induces Morphological Changes of $\mathrm{Pa}$}

Since we had observed that both SurA and to a lesser extent BamB have an impact on OM integrity of $P a$, we were interested if these changes result in obvious morphological changes. For this purpose, PA14 WT, the bamB and the conditional surA mutant strains grown in the presence or absence of arabinose were harvested, fixed in Karnovsky's fixative and visualized by transmission electron microscopy (Figure S1). The morphology of the PA14 WT strain was characterized by regular-shaped cells with a continuous, plain surface without any vesicles or protrusions attached. The BamB-deficient strain very much resembled the phenotype of a corresponding $Y e$ mutant strain (Weirich et al., 2017). It was characterized by numerous vesicles attached to the cell surface, probably a sign 

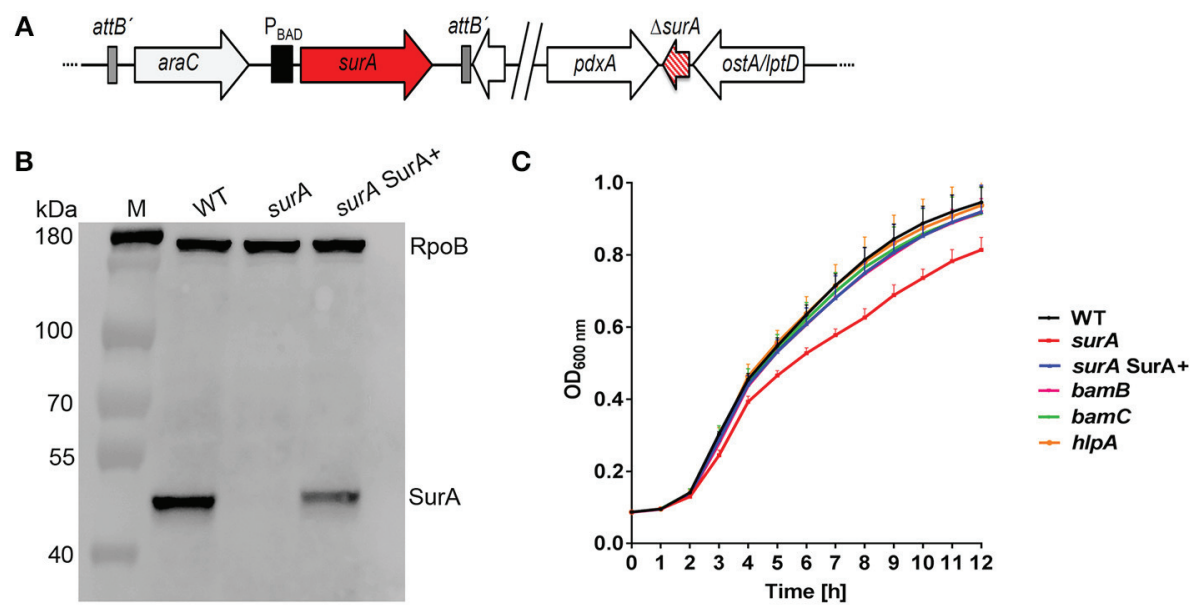

FIGURE 1 | Scheme of the conditional surA mutant, verification and impact of SurA, BamB, BamC and HipA on Pa growth. (A) Schematic view of the genomic organization of the conditional surA mutant. (B) Western blot analysis of SurA and RpoB of PA14 WT and the conditional surA mutant in the absence (surA) and presence of $0.2 \%$ arabinose (surA SurA+). (C) Growth curves of indicated strains. Data depict the mean and SD of at least 3 experiments. Growth curve of the conditional surA mutant is highlighted in red. ANOVA analyses revealed significant differences $(p<0.01)$ for both WT vs surA and surA SurA+ vs surA in the time range between 6 and $12 \mathrm{~h}$

A

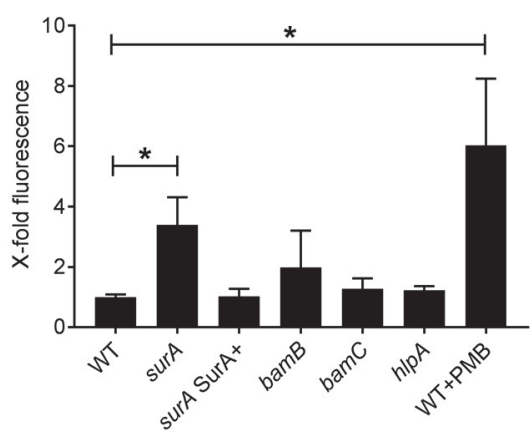

B

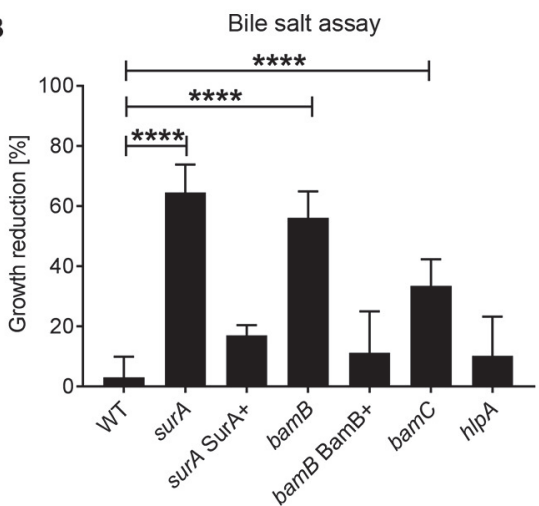

FIGURE 2 | Role of SurA, BamB, BamC, and HipA for membrane integrity and sensitivity against bile salts. (A) NPN Assay. A conditional surA and bamB, bamC, and h/pA deletion mutants were treated with NPN. Data depict the mean and SD of 3-5 independent experiments with triplicates. The fluorescence signal derived from matched numbers of bacteria was compared to that of WT. Polymyxin B (PMB) was used as a positive control. Asterisks indicate significant differences ( $p<0.05)$ compared to WT using ANOVA analysis. (B) Bile Salt Assay. Growth of the indicated Pa strains was measured in the absence or presence of $0.3 \%$ bile salts after $8 \mathrm{~h}$. Data depict the mean and SD of the growth reduction in $0.3 \%$ bile salts in LB compared to LB alone of at least 3 independent experiments with duplicates. Asterisks indicate significant differences $\left({ }^{* \star * *} p<0.0001\right.$ or $\left.{ }^{\star} p<0.05\right)$ as analyzed by ANOVA analysis.

for envelope stress (Kulp and Kuehn, 2010). Cells of the SurA depletion strain grown in the absence of arabinose also appeared rather regular-shaped, however, they looked slightly bloated and had some vesicles attached to their surface. Taken together, both a $\operatorname{bamB}$ and a conditional surA mutant of $\mathrm{Pa}$ showed visible changes in cell morphology, which corroborates previous findings obtained with $Y e$.

\section{Depletion of SurA Results in a Drastically Altered Composition of OMPs}

To analyze the OMP composition, OM fractions of WT and mutants were prepared and semi-quantitative proteomic analysis was performed using tryptic in-gel digestion and LC-MS/MS analysis. The ratio of label-free quantification (LFQ) intensities between the mutants and the WT was calculated. All differences in $\log _{2}$ LFQ intensities with a false discovery rate (FDR) $<0.1$ were considered significant. A list of all significant alterations is found in Table S3 (S3A: OMPs, S3B: all proteins). For the raw data please refer to http://proteomecentral.proteomexchange. org/cgi/GetDataset with the dataset identifier PXD011849.

The deletion mutant strains for $h l p A$, bamC and bamB exhibited just minor changes. In the $h l p A$ deletion mutant, only HlpA was reduced in abundance, as it was no longer detectable in the $\mathrm{OM}$ fraction. The bam $C$ deletion led to a significant reduction only of OmpH. Deletion of $\operatorname{bamB}$ led to a reduction of quite 
a number of proteins (e.g. FecA, OprB, PlpD) also found to be reduced in the SurA-depleted strain, however these changes were not significant according to our selection criteria. The relatively mild alterations in the $\mathrm{OM}$ composition may explain the comparably weak phenotypes of the $h l p A, \operatorname{bam} C$ and bamB deletion mutants with regards to OM integrity.

More interesting were the effects observed for SurA: depletion of SurA significantly altered the level of 42 proteins predicted to be localized in the OM (Table 1). Essentially, three groups could be differentiated: (i) proteins highly abundant in the $\mathrm{OM}$ of the WT but not detectable in the OM fraction of the conditional surA mutant (ratio surA/WT $<0.01$ ). This group included TonBdependent receptors and the siderophore receptors FpvA, FiuA and FecA, and Type V secretion systems (autotransporters). (ii) Proteins highly abundant in the OM fraction of WT and significantly reduced more than 3 -fold in the OM fraction of surA. This group included proteins of the BAM complex and porins (e.g., OprD, OprF, OprH). Finally (iii) a small group of proteins that showed higher protein levels in the OM fraction of the conditional surA mutant (e.g., OprM, OpmG, OpmB) compared to the WT.

In order to find out if the changes in protein abundance were caused on the transcriptional level, we assessed the relative mRNA levels of selected genes from the different functional groups of OMPs of the SurA depletion strain (grown exactly as for the mass spectrometry analyses) by quantitative RT-PCR and compared to the WT (Figure S2). From the genes tested, elevated amounts of mRNA transcripts were only found for $h l p A$ (2.4-fold), which might be a regulatory effect to compensate the reduced level of SurA. The transcriptional level of all other investigated genes was comparable for all WT, the conditional surA mutant and surA SurA+. These results indicate that the genes including the type Vd autotransporter PlpD (Salacha et al., 2010) and porins such as OprD seem to be true substrates of SurA and that their reduced abundance in the OM is probably the result of degradation within the periplasm.

\section{Validation of MS/MS Findings: Verification of Selected OMP Levels by Western Blot Analyses}

To further validate the proteomics data, the protein levels of SurA, OprD, and PlpD of the WT and the mutants were determined in whole cell lysates by Western blot analysis (Figures 3A,B). Comparable RpoB levels in all samples demonstrate equal loading of the lanes. Under depleting conditions (surA), no SurA was detectable by Western blot analysis demonstrating that the depletion worked well. Production of SurA in the surA SurA+ sample shows at least a partial recovery $(64 \%)$ compared to the PA14 WT strain. In accordance with the proteomics data (Table 1 and Figure $3 \mathrm{C}$ ), we found a decreased amount of OprD (15\%) and PlpD (24\%) in the whole cell lysate of the conditional surA mutant. As the PlpD antibody resulted in several bands in Western blot, a $p l p D$ deletion strain was employed to identify the band corresponding to $\mathrm{PlpD}$.

\section{Validation of MS/MS Findings: Impact of Reduced Siderophore Receptor Abundance}

As a consequence of the highly reduced levels of siderophore receptors (FpvA, FiuA, and FecA) under SurA-depleted conditions we assumed that the strain might suffer from a defective uptake of siderophore-iron complexes. Under ironrestricted conditions this should consequently lead to a growth reduction. Therefore, we assessed the growth characteristics of PA14 and the surA mutant under iron limitation. This was achieved by the addition of various amounts of the iron chelator $2,2^{\prime}$-Bipyridyl (BiP) to the growth medium (Figure S3). As assumed, under iron limitation $(+\mathrm{BiP})$, the SurA-depleted strain exhibited a significantly stronger $\mathrm{BiP}$ dose-dependent growth defect compared to the WT.

\section{Depletion of SurA Increases the Susceptibility for Killing by the Complement System}

An important first line host defense against invading bacteria specifically in bloodstream infection is the serum complement system. Therefore, we investigated whether serum resistance of $P a$ is altered in the (conditional) $\operatorname{sur} A, \operatorname{bamB}, \operatorname{bam} C$, and $h l p A$ mutants. To this end, serum killing tests using human serum were performed. The strains were incubated in $10 \%$ heat inactivated serum (HIS) or $10 \%$ normal human serum (NHS). Survival of bacteria was then quantified at indicated time points over a maximum period of $4 \mathrm{~h}$ (Figure $4 \mathrm{~A}$ ). While deletion of bamB, bam $C$ or hlpA had no impact on survival in active serum, the conditional surA mutant was killed rapidly when grown in the absence of arabinose (Figure 4B), indicating that the depletion of SurA alters the OM in a way that renders $\mathrm{Pa}$ highly susceptible to killing by the serum complement system.

\section{SurA Is Important for Virulence of $P a$ in the Galleria mellonella Infection Model}

To address the importance of the investigated genes for virulence, the Galleria mellonella infection model was used. For this purpose, $12 \pm 2$ cells of PA14 WT or the (conditional) surA, bamB, bamC or hlpA mutant were injected into the hemolymph of $G$. mellonella larvae. Thereafter, the survival of the larvae was monitored (Figure 5). Neither deletion of bamB, bamC, nor hlpA altered the survival compared to infection with the WT. However, infection with the conditional surA mutant led to a significant delay in the time to death. The conditional surA mutant was grown under two growth conditions prior to infection: (i) arabinose induced-SurA present prior to infection (SurA+) or (ii) uninduced-SurA absent prior to infection (SurA-). However, no significant difference was found between the survival curves of SurA+ and SurA-. This indicates that SurA production may decline rather quickly under in vivo conditions without continuous application of arabinose, which was not applicable in our experimental setting. Therefore, we could not test whether a complementation would fully rescue virulence. Nevertheless, our data demonstrate that SurA is critical for virulence of $\mathrm{Pa}$ in G. mellonella. 
TABLE 1 | Outer membrane proteins affected by SurA depletion.

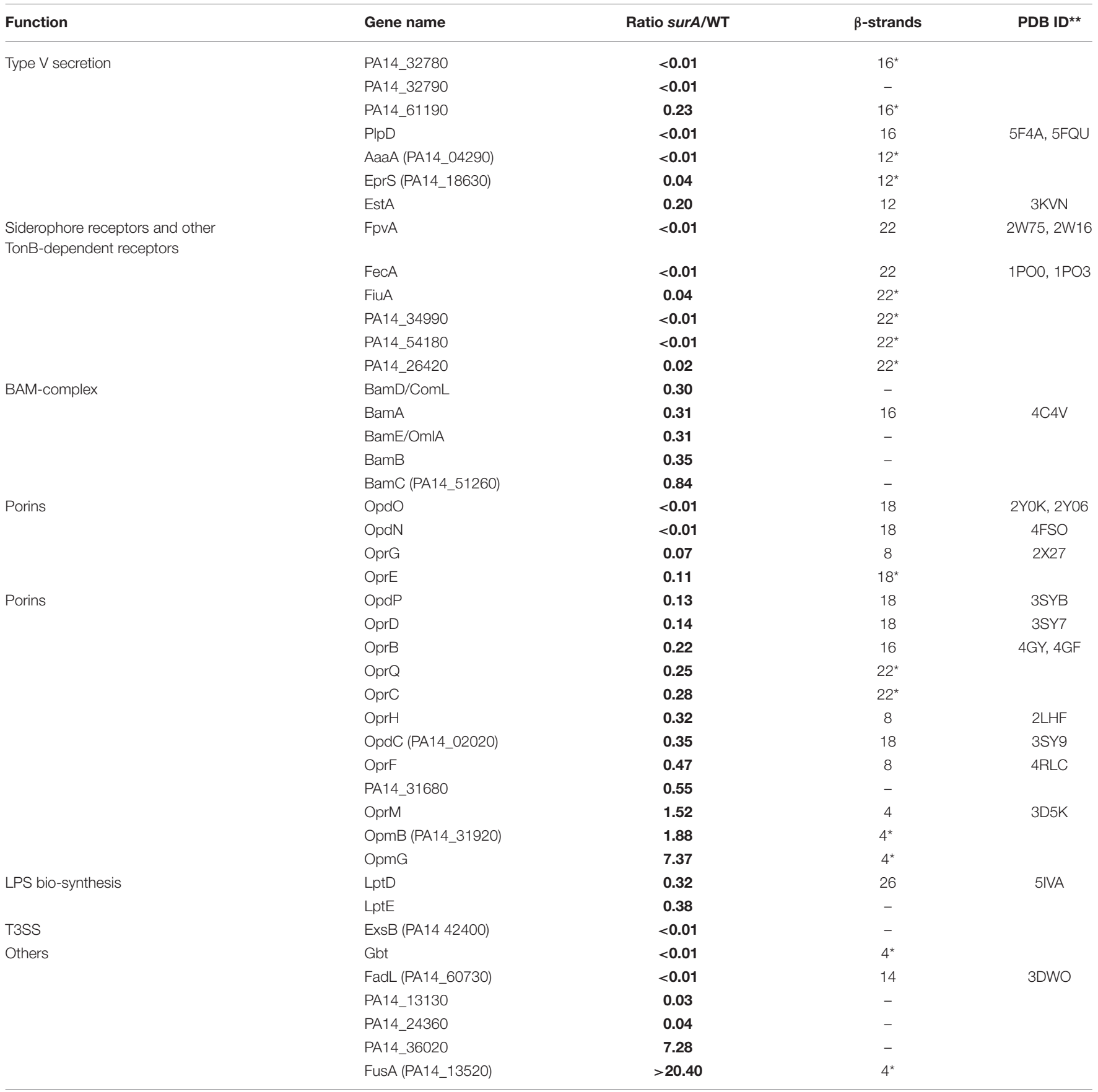

OM fractions of PA14 WT and the conditional surA mutant derived from three independent experiments were analyzed by mass spectrometry. Table depicts proteins which are described to be located in the OM and are significantly reduced or increased due to SurA depletion. Multiple t-testing was performed. Significant differences (FDR < 0.1) are shown in bold face. Number of $\beta$-strands of $\beta$-barrel proteins is indicated. " Predicted with Boctopus (Hayat and Elofsson, 2012); ${ }^{* *}$ Accession number of protein data bank (www.rcsb.org) of indicated proteins or orthologs.

\section{Susceptibility to Antibiotics}

The impermeability of the $\mathrm{OM}$ is the main reason that many antibiotics are not effective against Gram-negative bacteria, since they cannot pass the $\mathrm{OM}$ to reach their target. To investigate whether the depletion of SurA or BamB influences antibiotic susceptibility, we performed a comprehensive analysis with E-tests using the $\operatorname{bamB}$ deletion mutant, the conditional surA strains of PA14 and the clinical multidrug-resistant $\mathrm{Pa}$ bloodstream isolate ID72 (Willmann et al., 2018) [resistant against 3 classes out of the following: (I) 3rd and 4th generation cephalosporines (e.g., cefotaxim, ceftazidim), (II) acylureidopenicillins (e.g., piperacillin), (III) 

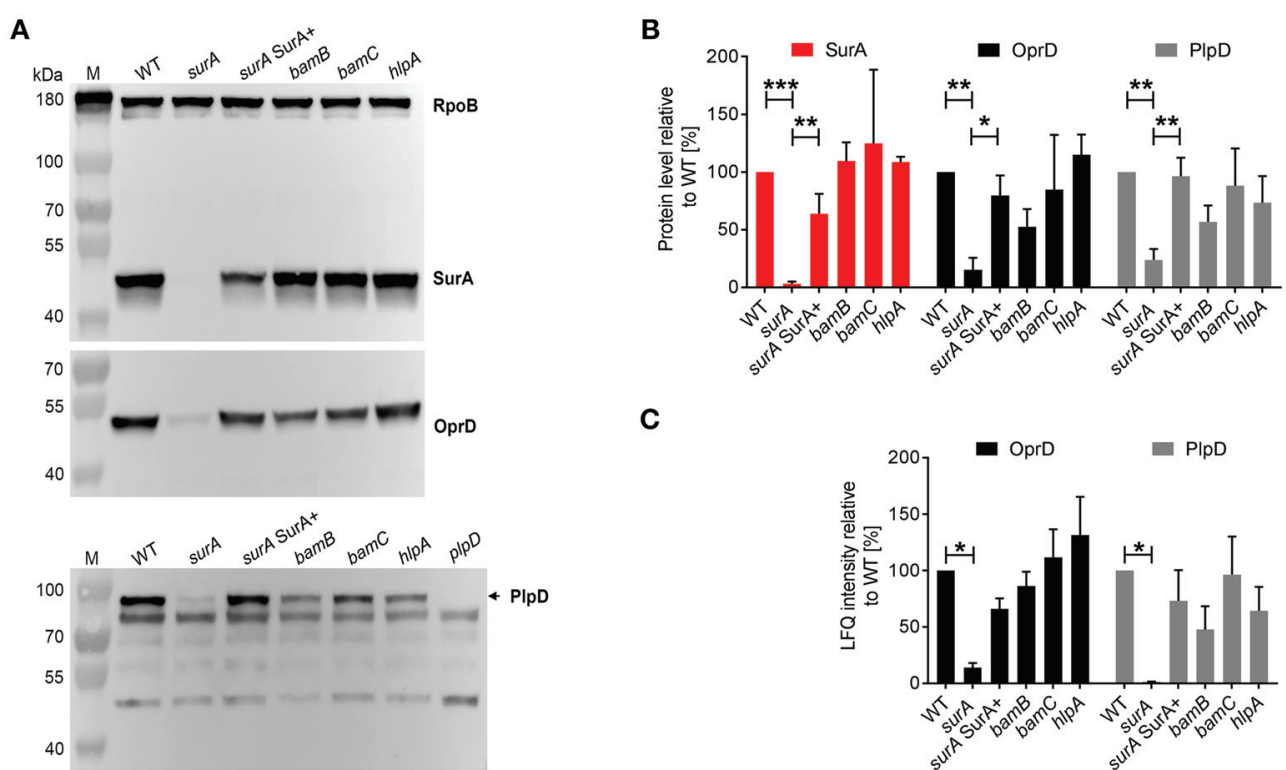

C

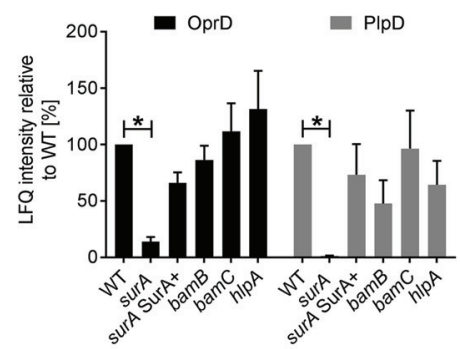

FIGURE 3 | Validation of proteome analysis by Western blot. (A) Comparison of protein levels between WT and mutants. Bacteria as indicated were sub-cultured for $3 \mathrm{~h}$ in the presence or absence of arabinose and samples were harvested for preparation of whole cell lysates. Western blot analysis was performed for RpoB, SurA OprD, and PlpD. (B) Quantification of immunoblots from 3 to 5 independent experiments using ImageJ software. Pixel intensity corresponds to protein levels. Asterisks indicate significant differences $\left({ }^{*} p<0.05,{ }^{* \star} p<0.01,{ }^{* \star *} p<0.001\right.$ ) between compared groups using ANOVA analysis. (C) Quantification of mass spectrometry analysis for OprD and PlpD. Graph indicates the LFQ intensity of OM fractions of indicated proteins. Asterisks indicate significant differences compared to WT by performing multiple $t$-tests with a FDR $<0.1(n=3)$.
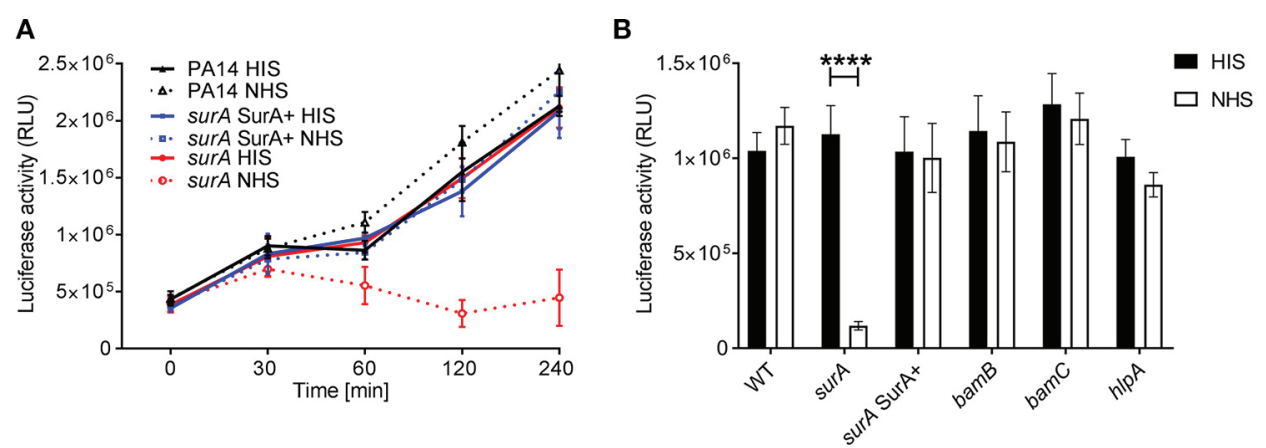

FIGURE 4 | SurA deletion leads to increased serum sensitivity. Indicated bacterial strains were grown for (A) various time periods or (B) $2 \mathrm{~h}$ and subsequently, luciferase activity (which is directly proportional to the ATP levels of viable cells in a sample) was measured. Data depict the mean and SD of luciferase activity measured of 3 independent experiments performed in triplicates. Asterisks indicate significant differences $(p<0.0001)$ analyzed by one way ANOVA analysis.

fluorchinolones (e.g., ciprofloxacin), and (IV) carbapenems (e.g., imipenem, meropenem)] (Figure S4) and the corresponding complemented strains compared to the WT control strains. Our test set additionally included several antibiotics not applicable for treatment of Gram-negative pathogens. However, these substances (vancomycin, erythromycin) can be used to detect $\mathrm{OM}$ defects in Gram-negatives (Wu et al., 2005). The deletion of $\operatorname{bamB}$ reduced the MIC values at least 4fold for ampicillin/sulbactam, ceftazidime, fosfomycin and vancomycin (Table 2). The complementation with arabinoseinduced $\mathrm{BamB}(\operatorname{bamB} \mathrm{BamB}+)$ restored the resistance against these antibiotics with the exception of fosfomycin. In summary, our data demonstrate that $\operatorname{bamB}$ deletion leads to a moderate increase in antibiotic susceptibility against several antibiotics.

Interestingly, for some of the tested antibiotics, we could observe at least a 4 -fold reduction of the MIC for both the PA14 and the ID72 conditional surA mutant. This was the case for ticarcillin/clavulanate (PA14 $32 \rightarrow 6 \mathrm{mg} / \mathrm{l}$; ID72 $>256 \rightarrow 64$ $\mathrm{mg} / \mathrm{l}$ ), ceftazidime (PA14 $2 \rightarrow 0.5 \mathrm{mg} / \mathrm{l} ;$ ID72 $>256 \rightarrow 8 \mathrm{mg} / \mathrm{l}$ ), levofloxacin (PA14 $0.38 \rightarrow 0.094 \mathrm{mg} / \mathrm{l}$; ID72 $1.5 \rightarrow 0.064 \mathrm{mg} / \mathrm{l}$ ), ciprofloxacin (PA14 0.19 $\rightarrow 0.038 \mathrm{mg} / \mathrm{l}$; ID72 $0.38 \rightarrow 0.064 \mathrm{mg} / \mathrm{l}$ ) and vancomycin $($ PA14 $>256 \rightarrow 12 \mathrm{mg} / \mathrm{l} ;$ ID72 $>256 \rightarrow 64 \mathrm{mg} / \mathrm{l})$. For the SurA-depleted strain in the PA14 background, we additionally observed a reduced MIC for ampicillin/sulbactam $($ PA14 $>256 \rightarrow 24 \mathrm{mg} / \mathrm{l})$. Moreover, the mutant in the ID72 


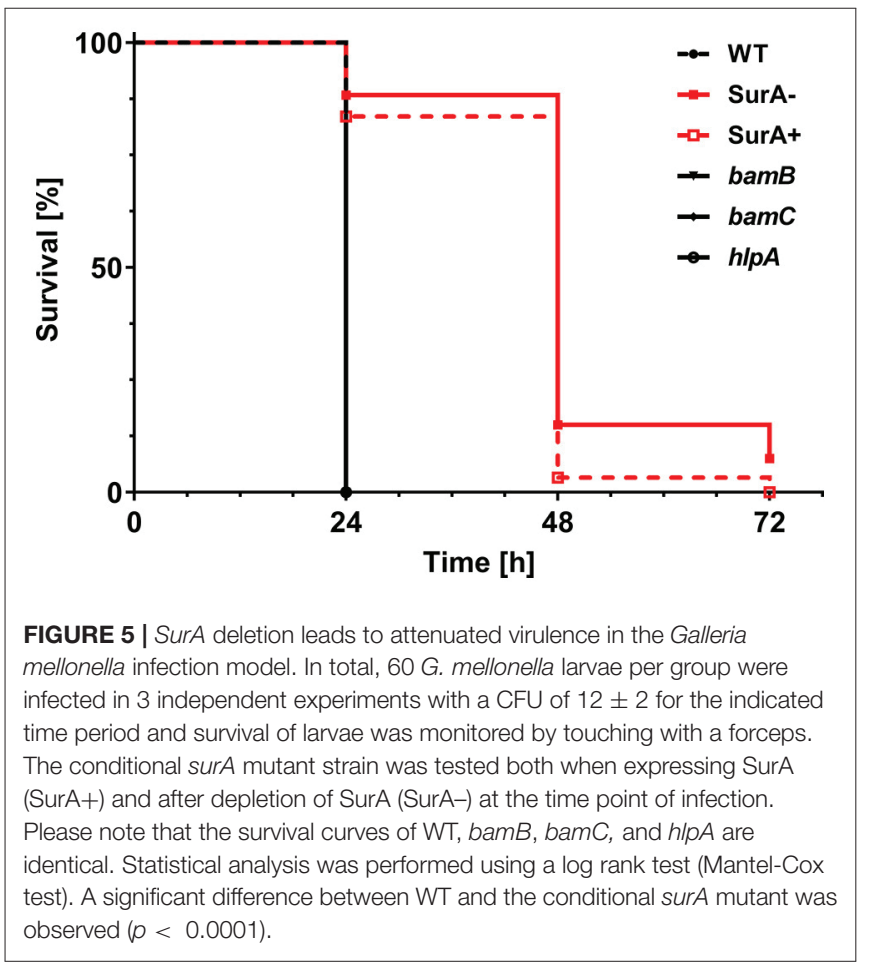

background displayed a reduced MIC for cefepime $(>32 \rightarrow 3$ $\mathrm{mg} / \mathrm{l})$. Strain-specific differences mediated by SurA depletion were found for ampicillin/sulbactam (increased sensitivity of PA14 surA but not ID72 surA) and cefepime (increased sensitivity of ID72 surA but not PA14 surA). Strikingly, in the SurA-depleted multidrug-resistant clinical bloodstream isolate ID72, the MIC values for cefepime, ceftazidime and levofloxacin were reduced to such an extent that according to the current EUCAST Clinical Breakpoint Tables (v. 8.1.), ID72 was re-sensitized to treatment with these antibiotics. In the case of ticarcillin/clavulanate, the MIC value was reduced. However, it did not drop below the critical breakpoint. Taken together, our data demonstrate that SurA depletion leads to an increased susceptibility against some representatives of clinically relevant antibiotics, even in the case of a multidrug-resistant $\mathrm{Pa}$ strain. Thus, SurA could possibly be used as a drug target to re-sensitize resistant strains to antibiotic therapy.

\section{DISCUSSION}

$\mathrm{Pa}$ is a difficult-to-treat pathogen and, compared to other Gramnegative bacteria, associated with a higher mortality that cannot be attributed to resistance only (Aloush et al., 2006; Willmann last resort antibiotic to defeat infections caused by $\mathrm{Pa}$, however, it has severe side effects and is rather nephrotoxic (Jeannot et al., 2017). Therefore, novel drugs and drug targets are required to control Pa infections (Perez et al., 2016).

The BAM complex and associated chaperones are responsible for the transport and insertion of the great majority of OMPs into the Gram-negative OM. Previous studies already highlighted et al., 2014; Thaden et al., 2017). Often colistin is considered as a

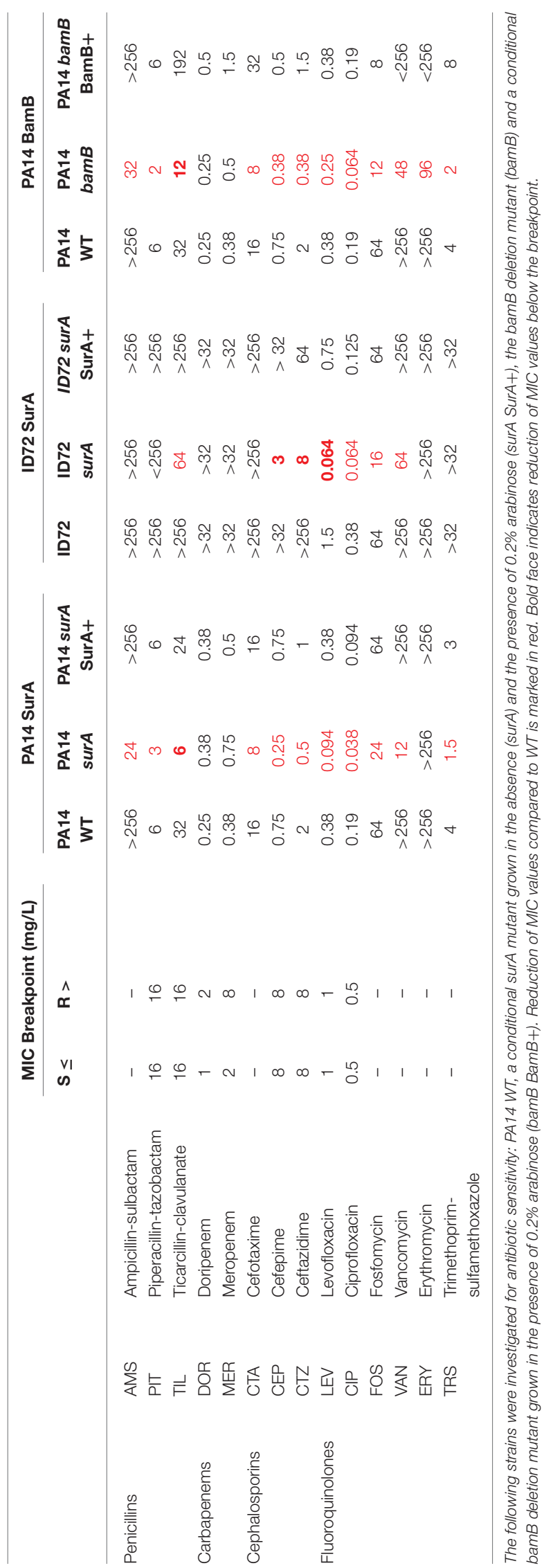

Frontiers in Microbiology | www.frontiersin.org 
the importance of the BAM complex as a putative drug target for several Gram-negative bacteria (Vertommen et al., 2009; Namdari et al., 2012; Hagan et al., 2015; Krachler, 2016; Weirich et al., 2017; Storek et al., 2018). The delivery of OMPs to the BAM complex is performed by the well-known chaperones SurA and Skp. Interestingly, according to the literature there are striking differences in the importance of these chaperones for OMP biogenesis. In $E c$ and $Y e$, SurA seems to play a major and Skp only a minor role for OMP biogenesis (Sklar et al., 2007). In contrast, in Neisseria meningitidis Skp but not SurA seems to play the major role for OMP biogenesis (Volokhina et al., 2011). According to the importance of $\mathrm{Pa}$ in clinical settings, we wanted to know which of the components of the BAM complex might be more useful as a target.

Therefore, we analyzed the role of distinct components of the BAM complex and the periplasmic chaperones HlpA/Skp and SurA for OM integrity and composition, virulence and antibiotic resistance. The main findings of this study are that depletion of SurA severely alters $\mathrm{Pa}$ OMP composition, which in consequence strongly influences $\mathrm{OM}$ integrity as well as resistance to bile salts, complement activity and antibiotics, which altogether leads to attenuated virulence and enhanced susceptibility to several antibiotics even in a multidrug-resistant bloodstream isolate of $\mathrm{Pa}$.

A comparably lower impact of the $\operatorname{bamB}$ deletion on $\mathrm{Pa}$ sensitivity against antimicrobial substances is perfectly in line with the milder phenotypes and minor changes in OMP composition of the bamB mutant. Similar findings have been made with Ec and Ye (Charlson et al., 2006; Weirich et al., 2017). Deletion of the $s k p$ homolog $h l p A$ and the BAM complex component bamC did not result in obvious phenotypes in our hands. In addition, none of these deletion mutants showed attenuation of virulence in the G. mellonella infection model. This is in line with previous studies on Skp in $E c$ where it was shown that Skp/HlpA may play only a minor role as chaperone to deliver OMPs to the BAM complex (Sklar et al., 2007).

Recently, it was asked whether BamB might be the achilles' heel for targeting Klebsiella pneumoniae (Kp) infection (Krachler, 2016). It was found that deletion of bamB led to a 15 -fold decrease in $K p$ adherence to retinal, intestinal and lung epithelial cells and consequently decreased invasion. $\operatorname{bamB}$ deletion had a pleiotropic effect on the profile of OMPs including a decrease of some porins as well as of type I fimbriae. Moreover, bamB deletion led to a significant attenuation of virulence in mice challenged intraperitoneally with $K p$ (Hsieh et al., 2016). Attenuation of virulence of a $\operatorname{bamB}$ deletion mutant was also found during $Y e$ infection (Behrens et al., 2001). In vitro assays showed increased sensitivity against antimicrobial components such as bile salts and complement activity. In addition, bamB deletion mutants of $Y e$ were sensitized to various antibiotics (typically not active against Gram-negative bacteria), such as vancomycin (Weirich et al., 2017). Like in Kp, several porins as well as the autotransporter invasin were significantly decreased in $Y e$. Another study addressing the role of BamB in $\mathrm{Pa}$ PAO1 already showed that bamB deletion also leads to sensitization against lysozyme, vancomycin and cefotaxime (Lee et al., 2017), which could be confirmed in our study. However, in contrast to $Y e$ or $K p$, neither increased sensitivity against human serum nor attenuation of virulence was observed. A common impact of bamB deletion in various species seems to be the reduction of the abundance of some porins (Malinverni et al., 2006; Hagan et al., 2010). In line with this, in the $\mathrm{Pa}$ bamB deletion mutant, porins such as OpdO (>93\% reduced) and OprB (45\% reduced) were found in lower levels in the OM. Some autotransporters like AaaA (67\% reduced) and PlpD (52\% reduced) were also found in lower levels in the OM. This is in agreement with previous studies, where it was observed that BamB-dependency of autotransporter proteins seemed to be correlated with the number of $\beta$-strands contained. Especially those proteins possessing a large number of $\beta$-strands were negatively affected by the absence of $\mathrm{BamB}$, whereas others were not (Rossiter et al., 2011; Weirich et al., 2017). However, these effects were rather moderate. Thus, BamB may contribute to the assembly of porins and autotransporters in $\mathrm{Pa}$, but in contrast to the function of BamB in $K p$ or $Y e$, the rather mild phenotypes we found upon deletion of $\operatorname{bamB}$ in $\mathrm{Pa}$ PA14 do not justify considering it as a promising target for drug development from our point of view. Nonetheless, given the results that have been obtained with e.g., $\mathrm{Pa}$ PAO1 and Salmonella (Namdari et al., 2012; Lee et al., 2017), it cannot be ruled out that the importance of BamB for OM composition and consequently the resulting phenotypes might vary significantly between strains and species.

The most interesting candidate as a putative drug target addressed in this study was found to be SurA. We recognized quite early during our studies that SurA might play an important role in $\mathrm{Pa}$ PA14, because it was not feasible to generate an inframe deletion mutant of surA. This indicated that surA might be essential in PA14, which would be in line with the findings of various other groups since there was no viable surA transposon mutant detected in their transposon libraries of different $\mathrm{Pa}$ strains (Skurnik et al., 2013; Lee et al., 2015; Turner et al., 2015) and also with own unpublished observations. Nevertheless, there is one transposon library in PA14 that contains three different mutants with transposons inserted into surA (Liberati et al., 2006). The transposon mutant with the ID38436 included in the available PA14NR set showed a similar phenotype like the conditional surA mutant in various assays and no SurA was detectable by Western blot analysis (data not shown). The insertion site of this mutant is located at the very beginning of the gene (at base pair 17), indicating inactivation of the gene. One possible explanation that this mutant is viable might be that compensatory mutations occurred in this transposon mutant. Altogether, we assume that SurA in $\mathrm{Pa}$ is essential in contrast to other Gram-negative bacteria. Nevertheless, the phenotypes observed in the SurA depletion strain of $\mathrm{Pa}$ are very similar to those of the deletion mutant in $Y e$ (Weirich et al., 2017).

While bamB deletion only leads to mild alteration in the OM composition, the depletion of SurA disturbed the insertion of a wide variety of OMPs of different functions, resulting in a drastically altered OM composition. Since the proper composition of the Gram-negative OM is important for its function as an impermeable barrier for many substances, it is reasonable that the reduced amount of several OMPs resulted in a higher permeability to the fluorescent dye NPN. 
The permeability barrier of the $\mathrm{OM}$ and the export of substances by efflux pumps are the main reasons for the high intrinsic resistance of $\mathrm{Pa}$ against many antibiotics (Nikaido, 1989; Poole, 2001). The reduced integrity of the OM could be an important reason, why the conditional surA mutants of PA14 and ID72 were better accessible to antibiotics such as vancomycin that are usually not able to cross the $\mathrm{OM}$ of $\mathrm{Pa}$ and reach their target inside the bacterial cell. Nevertheless, it cannot be excluded that other effects such as alteration in OMP composition or stress response may contribute to the increased antibiotic sensitivity. Thus, an inhibition of SurA could possibly permit a re-purposing of approved antimicrobials, currently active only against Grampositive pathogens, for use in Gram-negative bacteria. Of course this could work only if (i) the current limitation of use is a result of the inefficient entry and if (ii) the antimicrobial target is conserved and also present in the Gram-negative species. These data are in line with previous data found for the commensal $E c$ K12 as well as $Y e$ (Tamae et al., 2008; Weirich et al., 2017).

However, a critical precondition to consider SurA as a target specifically in species like $P a$ would be to break the resistance against therapeutically used antibiotics of multidrugresistant strains. By using a conditional ID72 surA mutant this could indeed be demonstrated for various antibiotics such as cephalosporins and fluoroquinolones.

In summary, from all the investigated factors, SurA was identified as the best target candidate to restore the sensitivity against some antibiotics by distortion of the OM specifically in multidrug-resistant strains. In the surA conditional mutant we found that the OM contained a higher amount of some single proteins like the OprM family porins OprM, OpmB and OpmG that are associated with the MexAB and MexXY efflux pumps (Poole, 2000). They are involved in mediating resistance against $\beta$-lactams, chloramphenicol, macrolides, quinolones and tetracycline (Li et al., 1995; Masuda et al., 2000), and aminoglycosides (Mao et al., 2001), respectively. Their increased abundance indicates that these porins are no dedicated substrates of SurA and their insertion into the OM may be facilitated in a different way, independent of SurA. OprM actually assembles into a trimer (Akama et al., 2004). It has been previously observed that a distinct subset of OMPs belonging to the TolC-like BAM substrates (i.e., multimeric with each monomer having only few $\beta$-strands) were affected only weakly by the absence of the non-essential Bam proteins and periplasmic chaperones. However, they were highly dependent on the essential Bam proteins BamA and BamD (Mahoney et al., 2016; Weirich et al., 2017). This might also apply to OprM family porins. Also the associated efflux pumps were found in a relatively higher amount in the $\mathrm{OM}$ of the conditional surA mutant, but this does not seem to influence its antibiotic sensitivity (Table 2).

With the exception of OprM, OpmG, and OpmB, many porins were detected in a significantly lower amount in the OM of the conditional surA mutant, including the most striking reduction observed for members of the OprD family (OpdO, OpdN, OpdP, and OprD). This may lead to a deprivation of nutrients, since most of these porins are specific transporters for different nutrients like pyroglutamate (OpdO), glycine-glutamate (OpdP), arginine (OprD and OprQ) and glucose (OprB) (Chevalier et al., 2017) and could also contribute to attenuation.

Besides the porins, also other groups of OMPs were strikingly affected by the depletion of SurA. We found that especially siderophore receptors and other TonB-dependent receptors (e.g., FpvA or FecA) (Pederick et al., 2015; Luscher et al., 2018) as well as different autotransporter proteins (e.g., PlpD or AaaA) were absent or less abundant in the OM upon depletion of SurA. The mRNA expression analysis suggested that the autotransporter protein PlpD is also a true substrate of SurA, similar to the autotransporter Inv of $Y e$ (Weirich et al., 2017). This means that these proteins are reduced in abundance because they cannot use any alternative insertion pathway when SurA is depleted. Thus they presumably are degraded by periplasmic proteases such as DegP (Sklar et al., 2007).

The finding that so many proteins involved in iron acquisition and transport were completely or almost completely absent in the conditional surA mutant, including the pyoverdine receptor FpvA, the ferric citrate transporter FecA and the ferrichrome receptor FiuA, suggests a reduced fitness of the conditional surA mutant under iron-limited conditions. This is in line with our findings that SurA depletion strongly affects growth in LB medium under iron-restricted conditions. In addition, it was previously shown that deletion of fiuA, besides its involvement in iron acquisition, leads to pleiotropic effects such as reduction of elastase levels and reduced virulence in an airway infection model (Lee et al., 2016). Therefore, the reduced abundance of siderophore receptors and the associated downstream effects could also contribute to attenuation of the SurA-depleted PA14 in the G. mellonella infection model.

Furthermore, the significantly reduced amount of the LptD/E complex (Chimalakonda et al., 2011) in the conditional surA mutant might result in an altered level of LptD in the OM (Lo Sciuto et al., 2018). The stable LptD/E complex is present at the OM and functions in the final stages of LPS assembly. The lipopolysaccharide transport (Lpt) is responsible for transporting LPS from the periplasmic side of the OM to the cell surface (Balibar and Grabowicz, 2016; Andolina et al., 2018). In line with previous studies (Vertommen et al., 2009; Weirich et al., 2017), LptD was shown to be a true substrate of SurA. Furthermore, it was shown that LptE depletion leads to reduced functionality of LptD resulting in impaired cell envelope integrity, reduced virulence and decreased antibiotic resistance (Lo Sciuto et al., 2018), which identifies LptD as a promising target for drug development. Actually, LptD is already addressed as a drug target by the macrocyle inhibitor Murepavadin (Polyphor POL7080), which is currently tested in a phase III clinical trial (MartinLoeches et al., 2018). This fact renders the concept of a SurA inhibitor -which is able to significantly reduce the cellular LptD protein levels- even more attractive.

The global changes in the OM composition of the conditional surA mutant including the reduced levels of many porins important for nutrient uptake, iron transport systems and proteins involved in LPS transport may in sum accumulate in reduced fitness. This is in line with the results of the G. mellonella infection model, since the larvae showed a prolonged time to death when infected with the conditional PA14 surA mutant. For 
the in vivo experiments, the leakiness of the araC- $\mathrm{P}_{\mathrm{BAD}}$ promoter (Meisner and Goldberg, 2016), still resulting in some mRNA expression, was actually a convenient feature: a partial reduction of SurA simulates the potential inhibition of the protein by a putative SurA inhibitor more realistically than a clean deletion.

Taken together, SurA is an important protein in $\mathrm{Pa}$ determining proper composition of the $\mathrm{OM}$ and seems to be an attractive target for an antiinfective drug. Its inhibition may lead to reduced fitness, may dampen multidrug resistance and could simultaneously render $\mathrm{Pa}$ accessible to various antibiotics that are usually not effective because of the OM barrier.

\section{DATA AVAILABILITY}

The dataset of the LC-MS/MS analysis for determination of OMP composition of the investigated bacterial strains can be found in the ProteomeXchange Consortium via the PRIDE (Vizcaino et al., 2016) partner repository with the dataset identifier PXD011849 (http://proteomecentral.proteomexchange. org/cgi/GetDataset, Username: reviewer54276@ebi.ac.uk, Password: i3rXLDrr).

\section{AUTHOR CONTRIBUTIONS}

The study was designed and supervised by EB, MS, and IA. Mass spectrometry and data analyses were performed by MF-W and BM. All other experimental data and analyses and generation

\section{REFERENCES}

Akama, H., Kanemaki, M., Yoshimura, M., Tsukihara, T., Kashiwagi, T., Yoneyama, H., et al. (2004). Crystal structure of the drug discharge outer membrane protein, OprM, of Pseudomonas aeruginosa: dual modes of membrane anchoring and occluded cavity end. J. Biol. Chem. 279, 52816-52819. doi: 10.1074/jbc.C400445200

Aloush, V., Navon-Venezia, S., Seigman-Igra, Y., Cabili, S., and Carmeli, Y. (2006). Multidrug-resistant Pseudomonas aeruginosa: risk factors and clinical impact. Antimicrob. Agents Chemother. 50, 43-48. doi: 10.1128/AAC.50.1. 43-48.2006

Andolina, G., Bencze, L. C., Zerbe, K., Müller, M., Steinmann, J., Kocherla, H., et al. (2018). A peptidomimetic antibiotic interacts with the periplasmic domain of LptD from Pseudomonas aeruginosa. ACS Chem. Biol. 13, 666-675. doi: 10.1021/acschembio.7b00822

Balibar, C. J., and Grabowicz, M. (2016). Mutant alleles of lptD increase the permeability of pseudomonas aeruginosa and define determinants of intrinsic resistance to antibiotics. Antimicrob. Agents Chemother. 60, 845-854. doi: 10.1128/AAC.01747-15

Behrens, S., Maier, R., De Cock, H., Schmid, F. X., and Gross, C. A. (2001). The SurA periplasmic PPIase lacking its parvulin domains functions in vivo and has chaperone activity. EMBO J. 20, 285-294. doi: 10.1093/emboj/20.1.285

Benjamini, Y., Krieger, A. M., and Yekutieli, D. (2006). Adaptive linear stepup procedures that control the false discovery rate. Biometrika 93, 491-507. doi: 10.1093/biomet/93.3.491

Borchert, N., Dieterich, C., Krug, K., Schutz, W., Jung, S., Nordheim, A., et al. (2010). Proteogenomics of Pristionchus pacificus reveals distinct proteome structure of nematode models. Genome Res. 20, 837-846. doi: $10.1101 /$ gr.103119.109

Charlson, E. S., Werner, J. N., and Misra, R. (2006). Differential effects of yfgL mutation on Escherichia coli outer membrane proteins and lipopolysaccharide. J. Bacteriol. 188, 7186-7194. doi: 10.1128/JB.00571-06 of tools were performed by KK, MSS, LF, TT, EB, MS, JL, and KL. The manuscript was written by KK, MSS, MS, and EB with contribution of all authors.

\section{FUNDING}

This work was supported by the German Research Council (DFG) under grant SFB 766 to IA and MS, the German Center of Infection Research (DZIF) under grant 06.801 to MS, and the Research Council of Norway, under grant 249793 to JL.

\section{ACKNOWLEDGMENTS}

The authors wish to thank Annika Schmidt and Sandra Schwarz for continuing support, Christiane Wolz for the introduction into qRT-PCR, Natalya Korn for excellent technical assistance in preparing RNA samples, Thilo Köhler (University of Geneva) for providing the OprD antibody, Francesco Imperi for providing

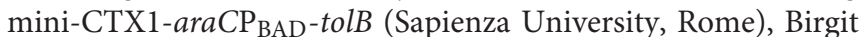
Fehrenbacher for the preparation of EM pictures and Matthias Willmann for providing the ID72 strain.

\section{SUPPLEMENTARY MATERIAL}

The Supplementary Material for this article can be found online at: https://www.frontiersin.org/articles/10.3389/fmicb. 2019.00100/full\#supplementary-material

Chevalier, S., Bouffartigues, E., Bodilis, J., Maillot, O., Lesouhaitier, O, Feuilloley, M. G. J., et al. (2017). Structure, function and regulation of Pseudomonas aeruginosa porins. FEMS Microbiol. Rev. 41, 698-722. doi: 10.1093/femsre/fux020

Chimalakonda, G., Ruiz, N., Chng, S. S., Garner, R. A., Kahne, D., and Silhavy, T. J. (2011). Lipoprotein LptE is required for the assembly of LptD by the beta-barrel assembly machine in the outer membrane of Escherichia coli. Proc. Natl Acad. Sci. U.S.A. 108, 2492-2497. doi: 10.1073/pnas. 1019089108

Cox, J., and Mann, M. (2008). MaxQuant enables high peptide identification rates, individualized p.p.b.-range mass accuracies and proteome-wide protein quantification. Nat. Biotechnol. 26, 1367-1372. doi: 10.1038/nbt.1511

Dunn, J. P., Kenedy, M. R., Iqbal, H., and Akins, D. R. (2015). Characterization of the beta-barrel assembly machine accessory lipoproteins from Borrelia burgdorferi. BMC Microbiol. 15:70. doi: 10.1186/s12866-015-0411-y

Elias, J. E., and Gygi, S. P. (2007). Target-decoy search strategy for increased confidence in large-scale protein identifications by mass spectrometry. Nat. Methods 4, 207-214. doi: 10.1038/nmeth1019

Epp, S. F., Pechere, J., and Kok, M. (2001). Raising antibodies against OprD, an outer membrane protein of Pseudomonas aeruginosa using translational fusions to MalE. J. Microbiol. Methods 46, 1-8. doi: 10.1016/S0167-7012(01)00236-6

Fardini, Y., Trotereau, J., Bottreau, E., Souchard, C., Velge, P., and VirlogeuxPayant, I. (2009). Investigation of the role of the BAM complex and SurA chaperone in outer-membrane protein biogenesis and type III secretion system expression in Salmonella. Microbiology 155, 1613-1622. doi: 10.1099/mic.0.025155-0

Gibson, D. (2009). One-step enzymatic assembly of DNA molecules up to several hundred kilobases in size. Protoc. Exch. doi: 10.1038/nprot.2009.77

Goemans, C., Denoncin, K., and Collet, J. F. (2014). Folding mechanisms of periplasmic proteins. Biochim. Biophys. Acta 1843, 1517-1528. doi: 10.1016/j.bbamcr.2013.10.014 
Goerke, C., Campana, S., Bayer, M. G., Doring, G., Botzenhart, K., and Wolz, C. (2000). Direct quantitative transcript analysis of the agr regulon of Staphylococcus aureus during human infection in comparison to the expression profile in vitro. Infect. Immunity 68, 1304-1311. doi: 10.1128/IAI.68.3.1304-1311.2000

Gu, Y., Li, H., Dong, H., Zeng, Y., Zhang, Z., Paterson, N. G., et al. (2016). Structural basis of outer membrane protein insertion by the BAM complex. Nature 531, 64-69. doi: 10.1038/nature17199

Hagan, C. L., Kim, S., and Kahne, D. (2010). Reconstitution of oute membrane protein assembly from purified components. Science 328, 890-892. doi: 10.1126/science.1188919

Hagan, C. L., Wzorek, J. S., and Kahne, D. (2015). Inhibition of the beta-barrel assembly machine by a peptide that binds BamD. Proc. Natl. Acad. Sci. U.S.A 112, 2011-2016. doi: 10.1073/pnas. 1415955112

Hayat, S., and Elofsson, A. (2012). BOCTOPUS: improved topology prediction of transmembrane beta barrel proteins. Bioinformatics 28, 516-522. doi: 10.1093/bioinformatics/btr710

Heuck, A., Schleiffer, A., and Clausen, T. (2011). Augmenting beta-augmentation: structural basis of how BamB binds BamA and may support folding of outer membrane proteins. J. Mol. Biol. 406, 659-666. doi: 10.1016/j.jmb.2011.01.002

Hoang, H. H., Nickerson, N. N., Lee, V. T., Kazimirova, A., Chami, M., Pugsley, A P., et al. (2011). Outer membrane targeting of Pseudomonas aeruginosa proteins shows variable dependence on the components of Bam and Lol machineries. mBio 2:e00246-11. doi: 10.1128/mBio.00246-11

Hoang, T. T., Kutchma, A. J., Becher, A., and Schweizer, H. P. (2000). Integrationproficient plasmids for Pseudomonas aeruginosa: site-specific integration and use for engineering of reporter and expression strains. Plasmid 43, 59-72. doi: 10.1006/plas.1999.1441

Hsieh, P. F., Hsu, C. R., Chen, C. T., Lin, T. L., and Wang, J. T. (2016) The Klebsiella pneumoniae $\mathrm{YfgL}$ (BamB) lipoprotein contributes to outer membrane protein biogenesis, type-1 fimbriae expression, anti-phagocytosis, and in vivo virulence. Virulence 7, 587-601. doi: 10.1080/21505594.2016.1171435

Jeannot, K., Bolard, A., and Plesiat, P. (2017). Resistance to polymyxins in Gram-negative organisms. Int. J. Antimicrob. Agents 49, 526-535. doi: 10.1016/j.ijantimicag.2016.11.029

Konovalova, A., Mitchell, A. M., and Silhavy, T. J. (2016). A lipoprotein/beta-barrel complex monitors lipopolysaccharide integrity transducing information across the outer membrane. Elife 5:e15276. doi: 10.7554/eLife.15276

Krachler, A. M. (2016). BamB and outer membrane biogenesis - the achilles' heel for targeting Klebsiella infections? Virulence 7, 508-511. doi: 10.1080/21505594.2016.1184388

Kulp, A., and Kuehn, M. J. (2010). Biological functions and biogenesis of secreted bacterial outer membrane vesicles. Annu. Rev. Microbiol. 64, 163-184. doi: 10.1146/annurev.micro.091208.073413

Lee, K., Lee, K. M., Go, J., Ryu, J. C., Ryu, J. H., and Yoon, S. S. (2016). The ferrichrome receptor A as a new target for Pseudomonas aeruginosa virulence attenuation. FEMS Microbiol. Lett. 363:fnw104. doi: 10.1093/femsle/fnw104

Lee, K. M., Lee, K., Go, J., Park, I. H., Shin, J. S., Choi, J. Y., et al. (2017). A genetic screen reveals novel targets to render Pseudomonas aeruginosa sensitive to lysozyme and cell wall-targeting antibiotics. Front. Cell. Infect. Microbiol. 7:59. doi: 10.3389/fcimb.2017.00059

Lee, S. A., Gallagher, L. A., Thongdee, M., Staudinger, B. J., Lippman, S. Singh, P. K., et al. (2015). General and condition-specific essential functions of Pseudomonas aeruginosa. Proc. Natl. Acad. Sci. U.S.A. 112, 5189-5194. doi: $10.1073 /$ pnas. 1422186112

Leyton, D. L., Rossiter, A. E., and Henderson, I. R. (2012). From self sufficiency to dependence: mechanisms and factors important for autotransporter biogenesis. Nat. Rev. Microbiol. 10, 213-225. doi: 10.1038/nrmicro2733

Li, G., He, C., Bu, P., Bi, H., Pan, S., Sun, R., et al. (2018). Single-molecule detection reveals different roles of Skp and SurA as chaperones. ACS Chem. Biol. 13, 1082-1089. doi: 10.1021/acschembio.8b00097

Li, X. Z., Nikaido, H., and Poole, K. (1995). Role of mexA-mexB-oprM in antibiotic efflux in Pseudomonas aeruginosa. Antimicrob. Agents Chemother. 39 1948-1953. doi: 10.1128/AAC.39.9.1948

Liberati, N. T., Urbach, J. M., Miyata, S., Lee, D. G., Drenkard, E., Wu, G., et al. (2006). An ordered, nonredundant library of Pseudomonas aeruginosa strain PA14 transposon insertion mutants. Proc. Natl. Acad. Sci. U.S.A. 103, 2833-2838. doi: 10.1073/pnas.0511100103

Lister, P. D., Wolter, D. J., and Hanson, N. D. (2009). Antibacterialresistant Pseudomonas aeruginosa: clinical impact and complex regulation of chromosomally encoded resistance mechanisms. Clin. Microbiol. Rev. 22, 582-610. doi: 10.1128/CMR.00040-09

Liu, H., and Naismith, J. H. (2008). An efficient one-step site-directed deletion, insertion, single and multiple-site plasmid mutagenesis protocol. BMC Biotechnol. 8:91. doi: 10.1186/1472-6750-8-91

Lo Sciuto, A., Fernandez-Pinar, R., Bertuccini, L., Iosi, F., Superti, F., and Imperi, F. (2014). The periplasmic protein TolB as a potential drug target in Pseudomonas aeruginosa. PLoS ONE 9:e103784. doi: 10.1371/journal.pone. 0103784

Lo Sciuto, A., Martorana, A. M., Fernandez-Pinar, R., Mancone, C., Polissi, A., and Imperi, F. (2018). Pseudomonas aeruginosa LptE is crucial for LptD assembly, cell envelope integrity, antibiotic resistance and virulence. Virulence 9, 1718-1733. doi: 10.1080/21505594.2018.15 37730

Luber, C. A., Cox, J., Lauterbach, H., Fancke, B., Selbach, M., Tschopp, J., et al. (2010). Quantitative proteomics reveals subset-specific viral recognition in dendritic cells. Immunity 32, 279-289. doi: 10.1016/j.immuni.2010. 01.013

Luscher, A., Moynie, L., Auguste, P. S., Bumann, D., Mazza, L., Pletzer, D., et al. (2018). TonB-dependent receptor repertoire of pseudomonas aeruginosa for uptake of siderophore-drug conjugates. Antimicrob. Agents Chemother. 62:e00097-18. doi: 10.1128/AAC.00097-18

Lyczak, J. B., Cannon, C. L., and Pier, G. B. (2000). Establishment of Pseudomonas aeruginosa infection: lessons from a versatile opportunist. Microbes Infect 2, 1051-1060. doi: 10.1016/S1286-4579(00)01259-4

Mahoney, T. F., Ricci, D. P., and Silhavy, T. J. (2016). Classifying beta-barrel assembly substrates by manipulating essential bam complex members. J. Bacteriol. 198, 1984-1992. doi: 10.1128/JB.00263-16

Malinverni, J. C., Werner, J., Kim, S., Sklar, J. G., Kahne, D., Misra, R., et al. (2006). YfiO stabilizes the YaeT complex and is essential for outer membrane protein assembly in Escherichia coli. Mol. Microbiol. 61, 151-164. doi: 10.1111/j.1365-2958.2006.05211.x

Mao, W., Warren, M. S., Lee, A., Mistry, A., and Lomovskaya, O. (2001). MexXY-OprM efflux pump is required for antagonism of aminoglycosides by divalent cations in Pseudomonas aeruginosa. Antimicrob. Agents Chemother. 45, 2001-2007. doi: 10.1128/AAC.45.7.2001-2007.2001

Martin-Loeches, I., Dale, G. E., and Torres, A. (2018). Murepavadin: a new antibiotic class in the pipeline. Expert Rev. Anti Infect. Ther. 16, 259-268. doi: 10.1080/14787210.2018.1441024

Masuda, N., Sakagawa, E., Ohya, S., Gotoh, N., Tsujimoto, H., and Nishino, T. (2000). Substrate specificities of MexAB-OprM, MexCD-OprJ, and MexXY-oprM efflux pumps in Pseudomonas aeruginosa. Antimicrob. Agents Chemother. 44, 3322-3327. doi: 10.1128/AAC.44.12.3322-3327. 2000

Meisner, J., and Goldberg, J. B. (2016). The Escherichia coli rhaSR-PrhaBAD inducible promoter system allows tightly controlled gene expression over a wide range in Pseudomonas aeruginosa. Appl. Environ. Microbiol. 82, 6715-6727. doi: 10.1128/AEM.02041-16

Merritt, M. E., and Donaldson, J. R. (2009). Effect of bile salts on the DNA and membrane integrity of enteric bacteria. J. Med. Microbiol. 58, 1533-1541. doi: $10.1099 / \mathrm{jmm} \cdot 0.014092-0$

Münzenmayer, L., Geiger, T., Daiber, E., Schulte, B., Autenrieth, S. E., Fraunholz, M., et al. (2016). Influence of Sae-regulated and Agr-regulated factors on the escape of Staphylococcus aureus from human macrophages. Cell. Microbiol. 18, 1172-1183. doi: $10.1111 / \mathrm{cmi} .12577$

Namdari, F., Hurtado-Escobar, G. A., Abed, N., Trotereau, J., Fardini, Y., Giraud, E., et al. (2012). Deciphering the roles of BamB and its interaction with BamA in outer membrane biogenesis, T3SS expression and virulence in Salmonella. PLoS ONE 7:e46050. doi: 10.1371/journal.pone.0046050

Necchi, F., Saul, A., and Rondini, S. (2017). Development of a highthroughput method to evaluate serum bactericidal activity using bacterial ATP measurement as survival readout. PLOS ONE 12:e0172163. doi: 10.1371/journal.pone. 0172163 
Nikaido, H. (1989). Outer membrane barrier as a mechanism of antimicrobial resistance. Antimicrob. Agents Chemother. 33, 1831-1836. doi: 10.1128/AAC.33.11.1831

Nikaido, H. (2003). Molecular basis of bacterial outer membrane permeability revisited. Microbiol. Mol. Biol. Rev. 67, 593-656. doi: 10.1128/MMBR.67.4.593-656.2003

Noinaj, N., Gumbart, J. C., and Buchanan, S. K. (2017). The beta-barre assembly machinery in motion. Nat. Rev. Microbiol. 15, 197-204. doi: $10.1038 /$ nrmicro.2016.191

Oberhettinger, P., Leo, J. C., Linke, D., Autenrieth, I. B., and Schutz, M. S. (2015) The inverse autotransporter intimin exports its passenger domain via a hairpin intermediate. J. Biol. Chem. 290, 1837-1849. doi: 10.1074/jbc.M114.604769

Ochsner, U. A., Vasil, A. I., Johnson, Z., and Vasil, M. L. (1999). Pseudomonas aeruginosa fur overlaps with a gene encoding a novel outer membrane lipoprotein, OmlA. J. Bacteriol. 181, 1099-1109.

Onufryk, C., Crouch, M. L., Fang, F. C., and Gross, C. A. (2005). Characterization of six lipoproteins in the sigmaE regulon. J. Bacteriol. 187, 4552-4561. doi: 10.1128/JB.187.13.4552-4561.2005

Page, W. J., and Taylor, D. E. (1988). Comparison of methods used to separate the inner and outer membranes of cell envelopes of Campylobacter spp. J. Gen. Microbiol. 134, 2925-2932. doi: 10.1099/00221287-134-11-2925

Pederick, V. G., Eijkelkamp, B. A., Begg, S. L., Ween, M. P., Mcallister, L. J., Paton, J. C., et al. (2015). ZnuA and zinc homeostasis in Pseudomonas aeruginosa. Sci. Rep. 5:13139. doi: 10.1038/srep13139

Pendleton, J. N., Gorman, S. P., and Gilmore, B. F. (2013). Clinical relevance of the ESKAPE pathogens. Exp. Rev. Anti Infect Ther. 11, 297-308. doi: 10.1586/eri.13.12

Perez, F., El Chakhtoura, N. G., Papp-Wallace, K. M., Wilson, B. M., and Bonomo, R. A. (2016). Treatment options for infections caused by carbapenem-resistant Enterobacteriaceae: can we apply "precision medicine" to antimicrobial chemotherapy? Exp. Opin. Pharmacother. 17, 761-781. doi: $10.1517 / 14656566.2016 .1145658$

Pfaffl, M. W. (2001). A new mathematical model for relative quantification in real-time RT-PCR. Nucleic Acids Res. 29:e45. doi: 10.1093/nar/29.9.e45

Poole, K. (2000). Efflux-mediated resistance to fluoroquinolones in gramnegative bacteria. Antimicrob. Agents Chemother. 44, 2233-2241. doi: 10.1128/AAC.44.9.2233-2241.2000

Poole, K. (2001). Multidrug efflux pumps and antimicrobial resistance in Pseudomonas aeruginosa and related organisms. J. Mol. Microbiol. Biotechnol. 3, 255-264

Rappsilber, J., Mann, M., and Ishihama, Y. (2007). Protocol for micropurification, enrichment, pre-fractionation and storage of peptides for proteomics using StageTips. Nat. Protoc. 2, 1896-1906. doi: 10.1038/nprot. 2007.261

Rice, L. B. (2008). Federal funding for the study of antimicrobial resistance in nosocomial pathogens: no ESKAPE. J. Infect. Dis. 197, 1079-1081. doi: $10.1086 / 533452$

Rietsch, A., Vallet-Gely, I., Dove, S. L., and Mekalanos, J. J. (2005). ExsE, a secreted regulator of type III secretion genes in Pseudomonas aeruginosa. Proc. Natl Acad. Sci. U.S.A. 102, 8006-8011. doi: 10.1073/pnas.05030 05102

Rolhion, N., Barnich, N., Claret, L., and Darfeuille-Michaud, A. (2005). Strong decrease in invasive ability and outer membrane vesicle release in Crohn's disease-associated adherent-invasive Escherichia col strain LF82 with the yfgL gene deleted. J. Bacteriol. 187, 2286-2296. doi: 10.1128/JB.187.7.2286-2296.2005

Rossiter, A. E., Leyton, D. L., Tveen-Jensen, K., Browning, D. F., Sevastsyanovich Y., Knowles, T. J., et al. (2011). The essential beta-barrel assembly machinery complex components $\mathrm{BamD}$ and BamA are required for autotransporter biogenesis. J. Bacteriol. 193, 4250-4253. doi: 10.1128/JB. 00192-11

Salacha, R., Kovacic, F., Brochier-Armanet, C., Wilhelm, S., Tommassen, J., Filloux, A., et al. (2010). The Pseudomonas aeruginosa patatin-like protein PlpD is the archetype of a novel Type V secretion system. Environ. Microbiol. 12, 1498-1512. doi: 10.1111/j.1462-2920.2010.02174.x

Schnaitman, C. A. (1971). Solubilization of the cytoplasmic membrane of Escherichia coli by Triton X-100. J. Bacteriol. 108, 545-552.
Sklar, J. G., Wu, T., Kahne, D., and Silhavy, T. J. (2007). Defining the roles of the periplasmic chaperones SurA, Skp, and DegP in Escherichia coli. Genes Dev. 21, 2473-2484. doi: 10.1101/gad.1581007

Skurnik, D., Roux, D., Aschard, H., Cattoir, V., Yoder-Himes, D., Lory, S., et al. (2013). A comprehensive analysis of in vitro and in vivo genetic fitness of Pseudomonas aeruginosa using high-throughput sequencing of transposon libraries. PLoS Pathog. 9:e1003582. doi: 10.1371/journal.ppat. 1003582

Storek, K. M., Auerbach, M. R., Shi, H., Garcia, N. K., Sun, D., Nickerson, N. N., et al. (2018). Monoclonal antibody targeting the beta-barrel assembly machine of Escherichia coli is bactericidal. Proc. Natl. Acad. Sci. U.S.A. 115, 3692-3697. doi: 10.1073/pnas.1800043115

Studier, F. W. (2005). Protein production by auto-induction in high density shaking cultures. Protein Expr. Purif. 41, 207-234. doi: 10.1016/j.pep.2005.01.016

Sydenham, M., Douce, G., Bowe, F., Ahmed, S., Chatfield, S., and Dougan, G. (2000). Salmonella enterica serovar typhimurium surA mutants are attenuated and effective live oral vaccines. Infect. Immunity 68, 1109-1115. doi: 10.1128/IAI.68.3.1109-1115.2000

Tamae, C., Liu, A., Kim, K., Sitz, D., Hong, J., Becket, E., et al. (2008). Determination of antibiotic hypersensitivity among 4,000 single-geneknockout mutants of Escherichia coli. J. Bacteriol. 190, 5981-5988. doi: 10.1128/JB.01982-07

Tashiro, Y., Sakai, R., Toyofuku, M., Sawada, I., Nakajima-Kambe, T., Uchiyama, H., et al. (2009). Outer membrane machinery and alginate synthesis regulators control membrane vesicle production in Pseudomonas aeruginosa. J. Bacteriol. 191, 7509-7519. doi: 10.1128/JB.00722-09

Thaden, J. T., Park, L. P., Maskarinec, S. A., Ruffin, F., Fowler, V. G. Jr., and Van Duin, D. (2017). Results from a 13-year prospective cohort study show increased mortality associated with bloodstream infections caused by Pseudomonas aeruginosa compared to other bacteria. Antimicrob. Agents Chemother. 61:e02671-16. doi: 10.1128/AAC.02671-16

Thein, M., Sauer, G., Paramasivam, N., Grin, I., and Linke, D. (2010). Efficient subfractionation of gram-negative bacteria for proteomics studies. J. Proteome Res. 9, 6135-6147. doi: 10.1021/pr1002438

Turner, K. H., Wessel, A. K., Palmer, G. C., Murray, J. L., and Whiteley, M. (2015). Essential genome of Pseudomonas aeruginosa in cystic fibrosis sputum. Proc. Natl. Acad. Sci. U.S.A. 112, 4110-4115. doi: 10.1073/pnas.1419677112

Vertommen, D., Ruiz, N., Leverrier, P., Silhavy, T. J., and Collet, J. F. (2009). Characterization of the role of the Escherichia coli periplasmic chaperone SurA using differential proteomics. Proteomics 9, 2432-2443. doi: 10.1002/pmic.200800794

Vij, R., Lin, Z., Chiang, N., Vernes, J. M., Storek, K. M., Park, S., et al. (2018). A targeted boost-and-sort immunization strategy using Escherichia coli BamA identifies rare growth inhibitory antibodies. Sci. Rep. 8:7136. doi: 10.1038/s41598-018-25609-z

Vizcaino, J. A., Csordas, A., Del-Toro, N., Dianes, J. A., Griss, J., Lavidas, I., et al. (2016). 2016 update of the PRIDE database and its related tools. Nucleic Acids Res. 44:11033. doi: 10.1093/nar/gkw880

Volokhina, E. B., Grijpstra, J., Stork, M., Schilders, I., Tommassen, J., and Bos, M. P. (2011). Role of the periplasmic chaperones Skp, SurA, and DegQ in outer membrane protein biogenesis in Neisseria meningitidis. J. Bacteriol. 193, 1612-1621. doi: 10.1128/JB.00532-10

Weirich, J., Bräutigam, C., Mühlenkamp, M., Franz-Wachtel, M., Macek, B., Meuskens, I., et al. (2017). Identifying components required for OMP biogenesis as novel targets for antiinfective drugs. Virulence 8, 1170-1188. doi: 10.1080/21505594.2016.1278333

Willmann, M., Goettig, S., Bezdan, D., Macek, B., Velic, A., Marschal, M., et al. (2018). Multi-omics approach identifies novel pathogen-derived prognostic biomarkers in patients with Pseudomonas aeruginosa bloodstream infection. biorxiv [Preprint]. doi: 10.1101/309898

Willmann, M., Klimek, A. M., Vogel, W., Liese, J., Marschal, M., Autenrieth, I. B., et al. (2014). Clinical and treatment-related risk factors for nosocomial colonisation with extensively drug-resistant Pseudomonas aeruginosa in a haematological patient population: a matched case control study. BMC Infect. Dis. 14:650. doi: 10.1186/s12879-014$0650-9$ 
Wu, T., Malinverni, J., Ruiz, N., Kim, S., Silhavy, T. J., and Kahne, D. (2005) Identification of a multicomponent complex required for outer membrane biogenesis in Escherichia coli. Cell 121, 235-245. doi: 10.1016/j.cell.2005. 02.015

Yorgey, P., Rahme, L. G., Tan, M. W., and Ausubel, F. M. (2001) The roles of mucD and alginate in the virulence of Pseudomonas aeruginosa in plants, nematodes and mice. Mol. Microbiol. 41, 1063-1076. doi: 10.1046/j.1365-2958.2001.02580.x

Yoshimura, F., and Nikaido, H. (1982). Permeability of Pseudomonas aeruginosa outer membrane to hydrophilic solutes. J. Bacteriol. $152,636-642$.
Conflict of Interest Statement: The authors declare that the research was conducted in the absence of any commercial or financial relationships that could be construed as a potential conflict of interest.

Copyright (C) 2019 Klein, Sonnabend, Frank, Leibiger, Franz-Wachtel, Macek, Trunk, Leo, Autenrieth, Schütz and Bohn. This is an open-access article distributed under the terms of the Creative Commons Attribution License (CC BY). The use, distribution or reproduction in other forums is permitted, provided the original author(s) and the copyright owner(s) are credited and that the original publication in this journal is cited, in accordance with accepted academic practice. No use, distribution or reproduction is permitted which does not comply with these terms. 
